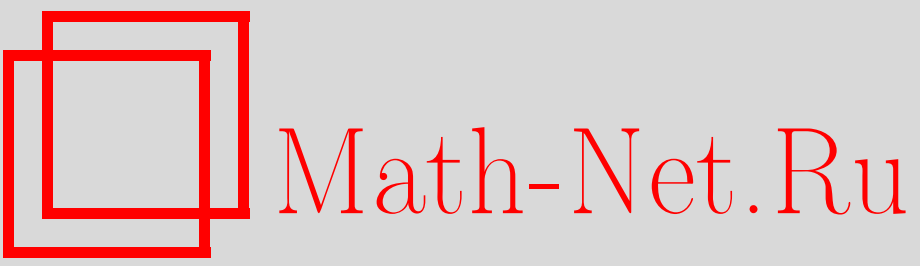

А. Н. Дранишников, Асимптотическая топология, УМН, 2000, том 55, выпуск $6,71-116$

DOI: https://doi.org/10.4213/rm334

Использование Общероссийского математического портала Math-Net.Ru подразумевает, что вы прочитали и согласны с пользовательским соглашением

http://www.mathnet.ru/rus/agreement

Параметры загрузки:

IP : 54.198 .55 .26

26 апреля 2023 г., $14: 45: 28$ 


\title{
АСИМПТОТИЧЕСКАЯ ТОПОЛОГИЯ
}

\author{
А.Н. ДрАнИшников
}

В работе рассматривается параллель между локальной топологией и ее глобальным аналогом, асимптотической топологией. В асимптотическом случае вводятся понятия размерности, когомологической размерности, абсолютных экстензоров и доказываются некоторые базисные факты о них. Рассматривается функтор взятия короны Хигсона, устанавливающий связь между макро- и микро-топологией. Обсуждается связь между проблемами общей асимптотической топологии и гипотезой Новикова о высших сигнатурах. Приводятся некоторые новые резултаты в направлении гипотезы Новикова и других родственных гипотез.

Библиография: 27 названий.

\section{СОДЕРЖАНИЕ}

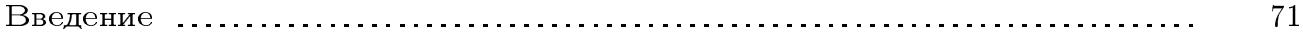

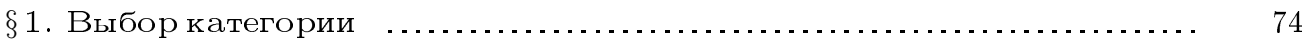

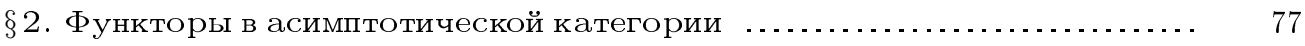

§ 3. Абсолютные экстензоры .................................... 81

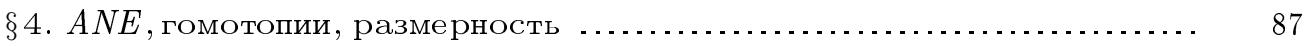

$\S 5$. Аппроксимация полиэдрами, когомологии и размерность .......... 92

$\S 6$. Корона Хигсона .......................................... 94

$\S 7$. Грубая гипотеза Новикова ................................ 100

$\S 8$. Принцип спуска и корона Хигсона ............................... 107

$\S 9$. Открытые проблемы ............................................ 112

Список литературы . . . . . . . . .

\section{Введение}

Асимптотическая геометрия, или, по-другому, - геометрия больших масштабов, стала очень популярным предметом в основном благодаря М. Громову. В настоящее время это основной инструмент изучения дискретных груп, а также инвариантов многообразий, ассоциированных с фундаментальной группой [1]-[3]. Асимптотическая геометрия изучает глобальные свойства (неограниченных) метрических пространств, например, полных открытых римановых многообразий, при этом мелкие (ограниченные) детали этих пространств не принимаются в расчет. Таким образом, изучаемые свойства или инварианты метрических пространств рассматриваются в 
пределе, на бесконечности. Мир больших масштабов - макромир - допускает аналогию с микромиром, где предел берется в нуле. Значительная часть топологии уделена изучению локальных свойств пространств и потому может быть отнесена к микромиру. Примерами могут служить: теория размерности, теория когомологической размерности, теория абсолютных окрестностных ретрактов $(A N R)$ и т. д. Основная цель настояшей работы - перенос вьшеуказанных теорий в макромир. Частично это уже сделано другими авторами. Так, М. Громов привел основные идеи макроскопической теории размерности [1], [4], а Дж. Ро дал определение макроскопических когомологий [2]. Перенос вышеуказанных теорий в макромир важен для многих областей математики. Так, Г. Ю [5] доказал, что гипотеза Новикова о высших сигнатурах выполняется для многообразий, у которых фундаментальная группа, рассматриваемая как метрическое пространство с метрикой слов, является конечномерной в макроскопическом смысле.

Многие свойства дискретных групп или универсальных накрьвающих пространств для этих групп могут быть ухвачены с помошњю границ групп. На данньй момент нет удовлетворительной конструкции гранищы дискретной группы. Возможно, такая конструкция просто не сушествует. М. Бествина привел аксиоматику для границы группы в [6], где отмечается, что во всех известных случаях граница может быть построена. Гранища единственна только для гиперболических груп, уже в случае САТ(0)-групш граница не есть инвариант квази-изометрий [7], [8]. С другой стороны, если допустить к рассмотрению неметризуемые пространства, то границу группы, инвариантную относительно квази-изометрий (и вообще любых изометрий в асимптотической топологии), построить можно. А именно, корона Хигсона обладает этим свойством. Так как корона Хигсона неметризуема, для ее изучения первостепенное значение имеют факторизационные теоремы типа Мардешича и спектральная теорема Щепина. Корона Хигсона есть функтор из макроскопического мира в мир компактных неметризуемых пространств. Помимо того, что геометрия компактных неметризуемых пространств чрезвычайно сложна, этот функтор имеет ряд других недостатков. Он не является инвариантом относительно гомотопий (в макроскопическом смысле). Так, например, $n$-мерное евклидово пространство $\mathbb{R}^{n}$ гомотопически эквивалентно в макроскопическом смысле $n$-мерному гиперболическому пространству $\mathbb{H}^{n}$, тогда как их короны имеют разные когомологии в размерности $n$ [9]. Тем не менее корона Хигсона является полезным инструментом при изучении гипотез типа гипотезы Новикова о высших сигнатурах.

Гипотеза Новикова о высших сигнатурах является одной из главных мотивировок для развития макроскопической топологии. Эта гипотеза утверждает, что высшие сигнатуры многообразия есть гомотопические инварианты. Для асферических многообразий гипотеза Новикова может быть сформулирована проще: рациональные классы Понтрягина суть гомотопические инварианты. Новиков доказал топологическую инвариантность рациональных классов Понтрягина [10]. В случае обших многообразий рациональные классы Понтрягина не являются гомотопическими инвариантами. Для односвязных многообразий $M$ сигнатура $\sigma(M)$ - единственный гомотопический инвариант, которьй может быть выражен через классы Понтрягина. Делается это с помощью формулы Хирцебруха:

$$
\left\langle L_{k}\left(P_{1}, \ldots, P_{k}\right),\left[M^{4 k}\right]\right\rangle=\sigma\left(M^{4 k}\right),
$$


где $L_{k}\left(P_{1}, \ldots, P_{k}\right)$ есть $k$-й полином Хирцебруха от классов Понтрягина, а $\left[M^{4 k}\right]-$ фундаментальньй гомологический класс многообразия $M^{4 k}$. Если размерность многообразия не делится на 4 , то сигнатура не определена (билинейная форма в когомологиях либо не определена, либо не является симметричной), тогда для односвязных многообразий $M$ гомотопических инвариантов в терминах понтрягинских классов просто не существует. При наличии же фундаментальной групшы $\Gamma=\pi_{1}(M) \neq 0$ сушествуют другие возможности. В этом случае помимо стандартного фундаментального класса $[M]$ сушествуют другие классы, претендуюшие на роль фундаментальных, классы, пришедшие из фундаментальной группы многообразия $M$. Интегрирование полиномов Хирцебруха по этим классам и определяет высшие сигнатуры.

Учитьвая вьшесказанное, можно говорить о гипотезе Новикова для данной группы Г. Из точной последовательности хирургии легко получить, что справедливость гипотезы Новикова для асферических многообразий влечет гипотезу Новикова для всех групा $\Gamma$, которые являются ретрактами фундаментальных групп асферических многообразий. Как доказал М. Дэвис, таковыми являются все группы Г с конечньм комплексом Эйленберга-Маклейна $K(\Gamma, 1)$ - так называемые геометрически конечные групшы. В случае геометрически конечных групп универсальное накрываюшее пространство $Е Г$ в асимптотическом смысле эквивалентно группе Г.

Другая серьезная гипотеза об асферических многообразиях - это гипотеза Громова-Лоусона [11]: асферическое многообразие не допускает римановой метрики с положительной скалярной кривизной. Связь между этой гипотезой и гипотезой Новикова обсуждается в [12], [13].

Пусть $X$ обозначает универсальное накрытие асферического многообразия $M^{n}$, снабженное метрикой, поднятой с $M^{n}$. Вышеупомянутые почтенные гипотезы могут быть сведены к вопросам о метрическом пространстве $X$. Как топологическое пространство $X$ есть стягиваемое $n$-многообразие. Следовательно, $X \times \mathbb{R}$ гомеоморффно $\mathbb{R}^{n+1}$. В вьшеуказанных гипотезах достаточно рассматривать случай, когда $X$ гомеоморфно $\mathbb{R}^{n}$. Все проблемы концентрируются в метрике на $\mathbb{R}^{n}$. В $\S 7$ настоящей статьи мы рассмотрим несколько утверждений о $X$, справедливость которых повлекла бы гипотезы Новикова и Громова-Лоусона.

Оказывается, что некоторые утверж дения обшей топологии, сформулированные на языке макроскопического мира, влекут гипотезы Новикова и Громова-Лоусона. Мы коснемся только утверж дений в теории размерности. Как уже упоминалось, Г. Ю [5] доказал, что если асимптотическая размерность геометрически конечной группы конечна, то гипотеза Новикова и гипотеза Громова-Лоусона вьполняются для многообразий с фундаментальной группой $Г$. В настоящей работе мы определяем когомологическую асимптотическую размерность метрических пространств $\operatorname{as~}_{\lim }^{\mathbb{Z}}$ и показьваем, что as $\operatorname{dim}_{\mathbb{Z}} \Gamma<\infty$ для всех геометрически конечных групп. Это сводит гипотезу Новикова к старой проблеме Александрова о совпадении лебеговой размерности и когомологической размерности, перенесенной в макромир. Проблема Александрова в классической теории размерности была решена контрпримером [14]. Контрпример был перенесен в макромир в работе [15], где была построена равномерно стягиваемая риманова метрика на $\mathbb{R}^{8}$ с бесконечной асимптотической размерностью и конечной когомологической асимптотической размерностью. Было бы интересно выяснить, для каких групп эти размерности совпадают. В случае компактных метрических пространств равенство между когомологической и лебеговой размерностью вытекает из 
представления компактных групп как обратных пределов групп Ли. Совсем не ясно, что компактные групшы являются аналогами дискретных групп при микро-макро аналогии. Возможно, что проблема Александрова для компактных $H$-пространств имеет более тесное отношение к нашему вопросу.

Статья разбита на девять параграфов. В $§ 1$ определяется категория, подходящая для нужд макроскопической топологии. Мы назьваем ее асимптотической категорией. Заметим, что наша категория немного отличается от грубой категории, определенной Дж. Ро. Различие обусловлено нашим желанием построить аналог теории $A N R$ в макроскопическом мире. В $\S 2$ мы приводим основные конструкции с объектами асимптотической категории. В $\S 3$ мы определяем абсолютные экстензоры и приводим простейшие примеры абсолютных экстензоров в асимптотической топологии. В $\S 4$ определяются окрестностные абсолютные экстензоры, вводится понятие гомотопии. Также обсуждаются различные определения макроскопической размерности. В частности, вводятся аналоги $n$-сфер и определяется грубая размерность в терминах продолжений морфизмов в грубые сферы. В 55 определяется античеховская аппроксимация асимптотических объектов полиэдрами, на основании этого определяются грубые когомологии и вводится понятие асимптотической когомологической размерности. В $\S 6$ определяется корона Хигсона. Затем различные асимптотические размерности, определенные в $\S 4$, сравниваются с лебеговой размерностью короны Хигсона. Основной результат здесь гласит, что размерность, определенная с помошью отображений в грубые сферы, совпадает с лебеговой размерностью короны Хигсона. В $\S 7$ мы доказываем некоторые версии грубой гипотезы Новикова при наличии некоторых теоретико-размерностных ограничений на групшы. Наши результаты продолжают результаты Г. Ю [5], [16]. В частности, мы доказываем грубую гипотезу Баума-Конна для групп с медленно растущей размерностью. В $\S 8$ мы сравниваем различные редукции (рациональной) гипотезы Новикова в терминах короны Хигсона. В $\S 9$ обсуждаются открытые проблемы.

\section{$\S$ 1. Выбор категории}

Метрическое пространство $(X, d)$ назьвается совериенным, если всякий замкнутый в нем шар $B_{r}\left(x_{0}\right)=\left\{x \in X \mid d\left(x, x_{0}\right) \leqslant r\right\}$ компактен. Напомним, что отображение $f: X \rightarrow Y$ называется совершенным, если прообраз $f^{-1}(C)$ компактен для всякого компактного множества $C \subset Y$. Заметим, что совершенное отображение между совершенными метрическими пространствами всегда непрерывно.

Отображение $f:\left(X, d_{X}\right) \rightarrow\left(Y, d_{Y}\right)$ назьвается асимптотически липиицевьм, если сушествуют два таких числа, $\lambda$ и $s$, что

$$
d_{Y}\left(f(x), f\left(x^{\prime}\right)\right) \leqslant \lambda d_{X}\left(x, x^{\prime}\right)+s .
$$

Асимптотическая категория $\mathscr{A}$ состоит из совершенных метрических пространств и совершенных асимптотически липшицевых отображений. Мы также рассматриваем следуюшую подкатегорию $\widetilde{\mathscr{A}}$. Пусть $x_{0} \in X$ будет фиксированной точкой, определим норму $\|x\|$ элемента $x \in X$ как расстояние $d_{X}\left(x, x_{0}\right)$. Затем определим норму отображения $\|f\|, f: X \rightarrow Y$, как $\lim _{\|x\| \rightarrow \infty}\|f(x)\| /\|x\|$. Очевидно, что для морфизмов категории $\mathscr{A}$ норма $\|f\|$ корректно определена и не зависит от выбора базисных точек $x_{0}$ и $y_{0}$. В категории $\widetilde{\mathscr{A}}$ мы рассматриваем морфизмы 
категории $\mathscr{A}$ с ненулевой нормой. Для любого морфизма $f: X \rightarrow Y$ в $\tilde{\mathscr{A}}$ найдутся константы $c$ и $b=b\left(x_{0}\right)$ такие, что $\|f(x)\| \geqslant c\|x\|-b$.

Примером морфизма в $\widetilde{\mathscr{A}}$ служит функция расстояния до данной точки $d\left(\cdot, x_{0}\right)$ : $X \rightarrow \mathbb{R}_{+}$. Заметим, что $d\left(\cdot, x_{0}\right)$ - сжимающее отображение.

ПРЕДЛОЖЕНИЕ 1.1. Для любого асимптотически липиицева отображения $f: X \rightarrow Y$ с константой Липшица $\lambda$ и для любого $\alpha>1$ cуществует $R>0$ такое, что $d_{Y}\left(f(x), f\left(x^{\prime}\right)\right)<\alpha \lambda d_{X}\left(x, x^{\prime}\right)$ для всех $x, x^{\prime}$ с $d\left(x, x^{\prime}\right) \geqslant R$.

Заметим, что изоморфизмами в категории $\tilde{\mathscr{A}}$ являются гомеоморфизмы $f: X \rightarrow Y$ такие, что $f$ и $f^{-1}$ асимптотически липшицевы. Изоморфизмы в $\tilde{\mathscr{A}}$ - это изоморфизмы в $\mathscr{A}$ с ненулевой нормой.

Пример 1 . Все $n$-мерные банаховы пространства изоморфны в $\tilde{\mathscr{A}} n$-мерному евклидову пространству $\mathbb{R}^{n}$.

Мы рассмотрим также большую категорию $\overline{\mathscr{A}}$, где объекты те же, что и в $\mathscr{A}$ (на самом деле можно рассматривать все метрические пространства), а морфизмы - асимптотически липшицевы грубо совершенные отображения. Последнее означает, что $f^{-1}(C)$ ограничено для любого ограниченного множества $C$. В этом случае отображение $f$ не обязательно непрерьвно. Грубая категория $\mathscr{C}$ является фактор-категорией категории $\overline{\mathscr{A}}$ по следуюшему отношению эквивалентности на морфизмах: два морфизма в $\overline{\mathscr{A}}$ являются грубо әквивалентными, если они находятся на конечном расстоянии друг от друга, т.е. для некоторой константы $c$ мы имеем $d_{Y}(f(x), g(x))<c$ для всех $x \in X$. Морфизм $f: X \rightarrow Y$ в $\overline{\mathscr{A}}$ называется грубимм изоморфизмом, если сушествует морфизм $g: Y \rightarrow X$ такой, что $f \circ g$ и $g \circ f$ эквивалентны тождественным отображениям $1_{X}$ и $1_{Y}$ соответственно. Метрические пространства $X$ и $Y$ являются грубо изоморфными (квази-изометричными), если сушествует грубый изоморфизм $f: X \rightarrow Y$.

Напомним, что расстояние по Громову-Хаусдорфу $d_{G H}(X, Y)$ между метрическими пространствами $X$ и $Y$ есть инфимум расстояний между множествами $i(X), j(Y) \subset$ $Z$ для всех возможных изометрических вложений $i, j$ и всевозможных метрических пространств $Z$. Обычно $d_{G H}(X, Y)=\infty$.

ПРЕДЛОЖЕНИЕ 1.2. Если $d_{G H}(X, Y)=\infty$, mо $X$ и $Y$ грубо изоморфны.

Заметим, что всякое отображение, находящееся на конечном расстоянии от асимптотически липшицева, само является асимптотически липшицевым.

ОПРЕДЕЛЕНИЕ. Метрическое пространство $X$ назьвается равномерно стягиваемыцм, $X \in U C$ (uniformly contractible), если существует функция $S: \mathbb{R}_{+} \rightarrow \mathbb{R}_{+}$такая, что всякий шар $B_{r}(x)$ радиуса $r$ с центром в $x$ стягивается в точку в шаре $B_{S(r)}(x)$.

ПрЕДЛОЖЕНИЕ 1.3. Для любъх объектов $X, Z$ из $\mathscr{A}$ с $X \in U C u \operatorname{dim} Z<\infty$ всякий морфизм $f: Z \rightarrow X$ из $\overline{\mathscr{A}}$ грубо изоморфен морфизму из $\mathscr{A}$.

ДокАЗАТЕльство. Пусть $\operatorname{dim} Z=n$. Предположим, что $\lambda$ и $s$ взяты из определения асимптотической липшицевости, примененного к $f$. Далее, пусть $S$ будет функцией из определения равномерной стягиваемости, примененного к $X$. Выберем покрытие $U$ пространства $Z$ кратности $\leqslant n+1$ и мелкости $\leqslant 1$. Пусть $\varphi: Z \rightarrow N$ означает проекцию на нерв покрытия $\mathscr{U}$. Определим отображение $g_{0}: N^{(0)} \rightarrow X$ на 0 -мерном остове 
комплекса $N$, задав $g_{0}(u)=f\left(x_{u}\right)$ для фиксированного $x_{u} \in \varphi^{-1}(u)$. Свойство $U C$ позволяет продолжить $g_{0}$ до отображения $g: N \rightarrow X$ таким образом, что $g\left(\sigma^{k}\right)$ для любого $k$-мерного симплекса $\sigma^{k}$ содержится в $S_{k}$-окрестности множества $g_{0}\left(\left(\sigma^{k}\right)^{(0)}\right)$, где $S_{k}=S(2 S(\ldots S(\lambda+s) \ldots))$ получено с помошью $k$ итераций. Для любой точки $z \in Z$ имеем:

$$
d(f(z), g \varphi(z)) \leqslant d\left(f(z), g_{0}(u)\right)+d\left(g_{0}(u), g \varphi(z)\right) .
$$

Возьмем $u$ таким, что $\varphi(z)$ и $u$ лежат в одном симплексе, тогда

$$
d(f(z), g \varphi(z)) \leqslant d\left(f(z), f\left(x_{u}\right)\right)+S_{n} \leqslant 1+S_{n}=c .
$$

Таким образом, $f$ находится на конечном расстоянии от $g \circ \varphi$. Легко видеть, что $g \circ \varphi$ - морфизм в $\mathscr{A}$.

ЗАмЕчАниЕ. В приведенном вьше доказательстве предложения 1.3 условие $\operatorname{dim} Z<\infty$ можно заменить более слабым: $Z$ допускает покрытие конечного порядка равномерно ограниченными множествами.

Дж. Ро [3] определил грубую категорию несколько по-другому. Вместо асимптотической липшицевости он требовал от отображений грубую равномерность. Напомним, что отображение метрических пространств $f: X \rightarrow Y$ является грубо равномернылм, если сушествует функция $S: \mathbb{R}_{+} \rightarrow \mathbb{R}_{+}$такая, что

$$
d_{X}\left(x, x^{\prime}\right) \leqslant r \Rightarrow d_{Y}\left(f(x), f\left(x^{\prime}\right)\right) \leqslant S(r) d_{X}\left(x, x^{\prime}\right) .
$$

Очевидно, что это свойство слабее асимптотической липшицевости. Напомним, что метрическое пространство $(X, d)$ называется геодезическим, если для любых двух точек $x, y \in X$ сушествует изометрическое вложение отрезка $j:[0, d(x, y)] \rightarrow X$, где $j(0)=x$, a $j(d(x, y))=y$.

ПРЕДЛОЖЕНИЕ 1.4. ДЛя геодезического метрического пространства $X$ всякое грубо равномерное отображение является асимптотически липшицевым.

ДокаЗАтельство. Возьмем $\lambda=s=S(1)$. Пусть $d\left(x, x^{\prime}\right)=n+\alpha, n \in \mathbb{Z}$, $0 \leqslant \alpha<1$. Тогда

$$
\begin{aligned}
d_{Y}\left(f(x), f\left(x^{\prime}\right)\right) & \leqslant d(f(x), f(j(1)))+\cdots+d(f(j(n-1)), f(j(n)))+d\left(f(j(n)), f\left(x^{\prime}\right)\right) \\
& \leqslant S(1) n+S(1)=\lambda n+s \leqslant \lambda d\left(x, x^{\prime}\right)+s
\end{aligned}
$$

где $j:\left[0, d\left(x, x^{\prime}\right)\right] \rightarrow X$ - геодезический сегмент, соединяющий $x$ с $x^{\prime}$.

Таким образом, при изучении геодезических метрических пространств нет разницы между нашими морфизмами и морфизмами в смысле Ро. Как мы увидим дальше, морфизмы Ро не допускают содержательной теории продолжения отображений (Extension Theory). Тем не менее есть ситуации, где морфизмы в смысле Ро работают лучше. Так, для них богаче теория вложений. Морфизм $j: Y \rightarrow X$ есть вложение в $\mathscr{A}$, если $j$ - инъекция и $j^{-1}:\left(j(Y),\left.d_{X}\right|_{j(Y)}\right) \rightarrow\left(Y, d_{Y}\right)$ есть морфизм в $\mathscr{A}$. Таким образом, наложение без растяжений и сжатий прямой на параболу, лежашую в $\mathbb{R}^{2}$, не является вложением $\mathbb{R}$ в $\mathbb{R}^{2}$ в категории $\mathscr{A}$. С другой стороны, это - вложение в смысле Ро. 


\section{§ 2. Функторы в асимптотической категории}

В этом параграфе мы переносим основные конструкции в топологии на категорию $\mathscr{A}$.

1. Произведение. Декартово произведение $X \times Y$ метрических пространств обладает естественной метрикой $d=d_{X}+d_{Y}$, которая эквивалентна "евклидовой" метрике $\sqrt{d_{X}^{2}+d_{Y}^{2}}$. С этой метрикой произведение совершенных метрических пространств будет совершенньм. Декартово произведение $\left(X \times Y, d_{X}+d_{Y}\right)$ не является категорньм в $\mathscr{A}$, так как проекции на сомножители не есть морфизмы. Мы определим асимптотическое произведение $X \widetilde{\times} Y$ как pull-back в топологической категории в диаграмме:

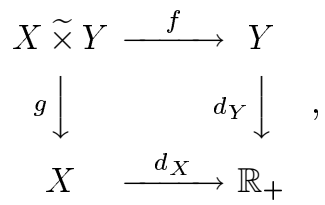

где $d_{X}(x)=d_{X}\left(x, x_{0}\right), d_{Y}(y)=d_{Y}\left(y, y_{0}\right)$, а $x_{0}, y_{0}$ - фиксированные точки в $X$ и $Y$. Метрика на $X \widetilde{\times} Y$ берется из естественного вложения $X \widetilde{\times} Y \subset X \times Y$. Это определение работает для пространств, у которых $d_{X}, d_{Y}-$ сюръекции. В общем случае pull-back может быть пустым множеством. Чтобы подчеркнуть, что $X \widetilde{\times} Y$ зависит от выбора $x_{0}, y_{0}$, мы будем иногда писать $X \widetilde{\times} Y\left(x_{0}, y_{0}\right)$.

ПРЕДЛОЖЕНИЕ 2.1. Для геодезических метрических пространств $X$ и $Y$ пространства $X \widetilde{\times} Y\left(x_{0}, y_{0}\right)$ и $X \widetilde{\times} Y\left(x_{0}^{\prime}, y_{0}^{\prime}\right)$ находятся на конечном расстоянии по Громову-Хаусдорфу для любого выбора точек $x_{0}, x_{0}^{\prime} \in X$ и $y_{0}, y_{0}^{\prime} \in Y$.

ДокАЗАтЕльство. Пусть $(x, y) \in X \widetilde{\times} Y\left(x_{0}, y_{0}\right)$. Тогдапо определению $d_{X}\left(x, x_{0}\right)$ $=d_{Y}\left(y, y_{0}\right)$. Возьмем $d=\min \left\{d_{X}\left(x, x_{0}\right), d_{X}\left(x, x_{0}^{\prime}\right), d_{Y}\left(y, y_{0}^{\prime}\right)\right\}$. Рассмотрим геодезические сегменты $\left[x_{0}^{\prime}, x\right]$ в $X$ и $\left[y_{0}^{\prime}, y\right]$ в $Y$ и возьмем точки $x^{\prime} \in\left[x_{0}^{\prime}, x\right]$ и $y^{\prime} \in\left[y_{0}^{\prime}, y\right]$ с $d_{X}\left(x_{0}^{\prime}, x^{\prime}\right)=d=d_{Y}\left(y_{0}^{\prime}, y^{\prime}\right)$. По определению $\left(x^{\prime}, y^{\prime}\right) \in X \widetilde{\times} Y\left(x_{0}^{\prime}, y_{0}^{\prime}\right)$. Заметим, что

$$
\begin{aligned}
d_{X \times Y}\left((x, y),\left(x^{\prime}, y^{\prime}\right)\right) & =d_{X}\left(x, x^{\prime}\right)+d_{Y}\left(y, y^{\prime}\right)=d_{X}\left(x, x_{0}^{\prime}\right)-d+d_{Y}\left(y, y_{0}^{\prime}\right)-d \\
& \leqslant\left|d_{X}\left(x_{0}^{\prime}, x\right)-d_{X}\left(x, x_{0}\right)\right|+\left|d_{Y}\left(y_{0}^{\prime}, y\right)-d_{Y}\left(y, y_{0}\right)\right| \\
& \leqslant d_{X}\left(x_{0}, x_{0}^{\prime}\right)+d_{Y}\left(y_{0}, y_{0}^{\prime}\right)=R .
\end{aligned}
$$

Мы использовали неравенство: $a+b-2 \min \{a, b, c\} \leqslant|a-c|+|b-c|$. Таким образом, $(x, y)$ лежит в $R$-окрестности множества $X \widetilde{\times} Y\left(x_{0}^{\prime}, y_{0}^{\prime}\right)$ в $X \times Y$. Симметрично можно показать, что $X \widetilde{\times} Y\left(x_{0}^{\prime}, y_{0}^{\prime}\right)$ лежит в $R$-окрестности множества $X \widetilde{\times} Y\left(x_{0}, y_{0}\right)$.

В силу предложения 1.2 класс пространства $X \widetilde{\times} Y$ в грубой категории не зависит от выбора базисных точек.

Как мы позже увидим, полупрямая $\mathbb{R}_{+}$играет роль точки в категории $\mathscr{A}$. Заметим, что $X \widetilde{\times} \mathbb{R}_{+}=X$ для любого $X$.

Аналог интервала $I=[0,1]$ в $\mathscr{A}$ есть полуплоскость $\mathbb{R}_{+}^{2}$. Мы определим вложение $J: X \widetilde{\times} \mathbb{R}_{+}^{2} \rightarrow X \times \mathbb{R}$ по формуле $J(x,(s, t))=(x, s)$, где $x \in X, t \in \mathbb{R}_{+}$, a $s \in \mathbb{R}$.

ПРЕДЛОЖЕНИЕ 2.2. J является вложением в категории $\mathscr{A}$. 
ДоказАтельство. Образ im $J$ при отображении $J$ есть область в $X \times \mathbb{R}$, зажатая между графиками функций $d_{X}$ и $-d_{X}$. Достаточно показать, что отображение $J: X \widetilde{\times} \mathbb{R}_{+}^{2} \rightarrow \operatorname{im} J$ билипшицево. Заметим, что

$$
\begin{aligned}
d_{X \times \mathbb{R}}\left(J(x,(s, t)), J\left(x^{\prime},\left(s^{\prime}, t^{\prime}\right)\right)\right) & =d_{X}\left(x, x^{\prime}\right)+\left|s-s^{\prime}\right|+\left|t-t^{\prime}\right| \\
& =d_{X \times \mathbb{R}_{+}^{2}}\left((x,(s, t)),\left(x^{\prime},\left(s^{\prime}, t^{\prime}\right)\right)\right) .
\end{aligned}
$$

Здесь нам удобнее рассматривать $l_{1}$-метрику на $\mathbb{R}_{+}^{2}$. С другой стороны, так как $\|x\|=$ $t+|s|$ и $\left\|x^{\prime}\right\|=t^{\prime}+\left|s^{\prime}\right|$, имеем

$$
\begin{aligned}
2 d_{X \times \mathbb{R}_{+}}\left(J(x,(s, t)), J\left(x^{\prime},\left(s^{\prime}, t^{\prime}\right)\right)\right) & =2 d_{X}\left(x, x^{\prime}\right)+2\left|s-s^{\prime}\right| \\
& \geqslant d_{X}\left(x, x^{\prime}\right)+\left|\|x\|-\left\|x^{\prime}\right\|\right|+|| s|-| s^{\prime}||+\left|s-s^{\prime}\right| \\
& \geqslant d_{X}\left(x, x^{\prime}\right)+\left|t-t^{\prime}\right|+\left|s-s^{\prime}\right| \\
& =d_{X \times \mathbb{R}_{+}^{2}}\left((x,(s, t)),\left(x^{\prime},\left(s^{\prime}, t^{\prime}\right)\right)\right) .
\end{aligned}
$$

Мы определим два вложения $i_{ \pm}: X \rightarrow X \widetilde{\times} \mathbb{R}_{+}^{2}$ по формуле $i_{ \pm}(x)=J^{-1}(x, \pm\|x\|)$. Легко проверить следующее

ПРЕДЛОЖЕНИЕ 2.3. Отображения $i_{+}, i_{-}$являются вложениями в категории $\widetilde{\mathscr{A}}$ и, следовательно, в $\mathscr{A}$.

Для метрических пространств $X$ и $Y$ с базисными точками $x_{0}, y_{0}$ мы определим букет $X \vee Y$ как топологический букет, наделенньй метрикой $d(x, y)=d_{X}\left(x, x_{0}\right)+$ $d_{Y}\left(y_{0}, y\right)$ для $x \in X$ и $y \in Y$, причем $\left.d\right|_{X}=d_{X}$ и $\left.d\right|_{Y}=d_{Y}$.

2. Фактор-пространства. Рассмотрим совершенное сюръективное отображение метрического пространства $f: X \rightarrow Y$. Мы определим метрику $d_{Y}$ на $Y$ таким образом, что $f$ будет морфизмом. Положим

$$
s_{Y}\left(y, y^{\prime}\right)=d_{X}\left(f^{-1}(y), f^{-1}\left(y^{\prime}\right)\right)=\inf \left\{d_{X}\left(x, x^{\prime}\right) \mid x \in f^{-1}(y), x^{\prime} \in f^{-1}\left(y^{\prime}\right)\right\} .
$$

Определим метрику $d_{Y}$ как внутреннюю метрику, порожденную функцией $s_{Y}$ :

$$
d_{Y}\left(y, y^{\prime}\right)=\inf \left\{\sum_{i=1}^{n} s_{Y}\left(y_{i}, y_{i+1}\right) \mid n \in \mathbb{N} ; y_{1}, \ldots, y_{n} \in Y, y_{1}=y, y_{n}=y^{\prime}\right\}
$$

Таким образом, $d_{Y}\left(f(x), f\left(x^{\prime}\right)\right) \leqslant s_{Y}\left(f(x), f\left(x^{\prime}\right)\right) \leqslant d_{X}\left(x, x^{\prime}\right)$, и, следовательно, $f-$ сжимающее отображение.

Пространство $Y$ с метрикой $d_{Y}$ мы называем фактор-пространством, а $d_{Y}-\oint a \kappa-$ тор-метрикой.

Для любого совершенного метрического пространства $X$ и его замкнутого подмножества $A$ через $X / A$ мы обозначим фактор-пространство, полученное из отображения $f: X \rightarrow X \cup_{d} \mathbb{R}_{+}$, индуцированного отображением $d: A \rightarrow \mathbb{R}_{+}, d\left(\cdot, x_{0}\right)$. Заметим, что в топологической категории $X / A$ определяется с помошью постоянного отображения $d: A \rightarrow \mathrm{pt}$, а в категории $\mathscr{A}$ постоянное отображение заменяется функцией расстояния. 
3. Конус и надстройка. Так как у нас уже имеется аналог единичного интервала, $\mathbb{R}_{+}^{2}$, а также есть понятие фактор-пространства, то мы можем определить конус $C X$ и надстройку $\Sigma X$ для любого метрического пространства по аналогии: $C X=X \widetilde{\times} \mathbb{R}_{+}^{2} / i_{+}(X)$ и $\Sigma X=X \widetilde{\times} \mathbb{R}_{+}^{2} / i_{ \pm}(X)=C X / i_{-}(X)$. Конструкция конуса и надстройки предлагает простые формулы $C X=X \times \mathbb{R}_{+}$и $\Sigma X=X \times \mathbb{R}$ для разумных пространств $X$, скажем, для геодезических. Мы докажем эти формулы для $X=\mathbb{R}^{n}$.

ЛЕмма 2.4. Конус $C \mathbb{R}^{n}$ изоморфен полупространству $\mathbb{R}_{+}^{n+1}$.

ДокАЗАТЕльСтво. Пусть $q: \mathbb{R}^{n} \widetilde{\times} \mathbb{R}_{+}^{2} \rightarrow C \mathbb{R}^{n}$ означает фактор-отображение. Для простоты мы отождествим $\mathbb{R}^{n} \tilde{\times} \mathbb{R}_{+}^{2} \mathrm{cim} J \cap\left(\mathbb{R}^{n} \times \mathbb{R}_{+}\right)$. Определим отображение $T: C \mathbb{R}^{n} \rightarrow \mathbb{R}^{n} \times \mathbb{R}_{+}$формулой

$$
T(q(x, t))=\left(\frac{\|x\|-t}{\|x\|} x, t\right) .
$$

В таком случае обратное отображение $T^{-1}$ задается формулой

$$
T^{-1}(y, t)=q\left(\frac{\|y\|+t}{\|y\|} y, t\right) .
$$

Сначала мы покажем, что обратное отображение $T^{-1}$ липшицево. Обозначим через $d l_{1}$-метрику $d_{\mathbb{R}^{n}} \times \mathbb{R}_{+}$. Тогда

$$
\begin{aligned}
d_{C \mathbb{R}^{n}}\left(T^{-1}(y, t), T^{-1}\left(y^{\prime}, t^{\prime}\right)\right) & =d_{C \mathbb{R}^{n}}\left(q\left((\|y\|+t) \frac{y}{\|y\|}, t\right), q\left(\left(\left\|y^{\prime}\right\|+t^{\prime}\right) \frac{y^{\prime}}{\left\|y^{\prime}\right\|}, t^{\prime}\right)\right) \\
& \leqslant d\left(\left((\|y\|+t) \frac{y}{\|y\|}, t\right),\left(\left(\left\|y^{\prime}\right\|+t^{\prime}\right) \frac{y^{\prime}}{\left\|y^{\prime}\right\|}, t^{\prime}\right)\right) \\
& =\left\|(\|y\|+t) \frac{y}{\|y\|}-\left(\left\|y^{\prime}\right\|+t^{\prime}\right) \frac{y^{\prime}}{\left\|y^{\prime}\right\|}\right\|+\left|t-t^{\prime}\right| .
\end{aligned}
$$

Не теряя обшности, можно предположить, что $\left\|y^{\prime}\right\| \leqslant\|y\|$. Тогда неравенство можно продолжить:

$$
\begin{aligned}
\leqslant & \left\|(\|y\|+t) \frac{y}{\|y\|}-(\|y\|+t) \frac{y^{\prime}}{\|y\|}\right\| \\
& +\left\|\left(1+\frac{t}{\|y\|}\right) y^{\prime}-\left(1+\frac{t^{\prime}}{\left\|y^{\prime}\right\|}\right) y^{\prime}\right\|+\left|t-t^{\prime}\right| \\
\leqslant & \left(1+\frac{t}{\|y\|}\right)\left\|y-y^{\prime}\right\|+\left\|y^{\prime}\right\|\left|\frac{t}{\|y\|}-\frac{t^{\prime}}{\left\|y^{\prime}\right\|}\right|+\left|t-t^{\prime}\right| \\
\leqslant & 2\left\|y-y^{\prime}\right\|+\left\|y^{\prime}\right\|\left|\frac{t}{\|y\|}-\frac{t^{\prime}}{\|y\|}\right| \\
& +\left\|y^{\prime}\right\|\left|\frac{t^{\prime}}{\|y\|}-\frac{t^{\prime}}{\left\|y^{\prime}\right\|}\right|+\left|t-t^{\prime}\right| \\
\leqslant & 2\left\|y-y^{\prime}\right\|+\left|t-t^{\prime}\right|+\frac{t^{\prime}}{\|y\|}\left|\|y\|-\left\|y^{\prime}\right\|\right|+\left|t-t^{\prime}\right| \\
\leqslant & 3\left\|y-y^{\prime}\right\|+3\left|t-t^{\prime}\right|=3 d\left((y, t),\left(y^{\prime}, t^{\prime}\right)\right) .
\end{aligned}
$$


Покажем, что $T$ липшицево с константой 3 , т.е.

$$
d\left(T q(x, t), T q\left(x^{\prime}, t^{\prime}\right)\right) \leqslant 3 d\left((x, t),\left(x^{\prime}, t^{\prime}\right)\right)
$$

для любых $(x, t),\left(x^{\prime}, t^{\prime}\right) \in \mathbb{R}^{n} \widetilde{\times} \mathbb{R}_{+}^{2} \subset \mathbb{R}^{n} \times \mathbb{R}_{+}$. Не теряя общности, предположим $\left\|x^{\prime}\right\| \geqslant\|x\|$. Рассмотрим два случая: 1$) t^{\prime} \leqslant\|x\|$ и 2) $t^{\prime} \geqslant\|x\|$. Заметим, что $d\left((x, t), i_{+}\left(\mathbb{R}^{n}\right)\right)=\|x\|-t$. Тогда

$$
\begin{aligned}
d_{C \mathbb{R}^{n}}\left(q(x, t), q\left(x^{\prime}, t^{\prime}\right)\right) & \geqslant \min \left\{d\left((x, t),\left(x^{\prime}, t^{\prime}\right)\right), d\left((x, t), i_{+}\left(\mathbb{R}^{n}\right)\right)+d\left(\left(x^{\prime}, t^{\prime}\right), i_{+}\left(\mathbb{R}^{n}\right)\right)\right\} \\
& =\min \left\{\left\|x-x^{\prime}\right\|+\left|t-t^{\prime}\right|,\|x\|-t+\left\|x^{\prime}\right\|-t^{\prime}\right\} .
\end{aligned}
$$

Мы покажем, что в первом случае выполняются неравенства:

$$
\begin{aligned}
3\left(\left\|x-x^{\prime}\right\|+\left|t-t^{\prime}\right|\right) & \geqslant d\left(T q(x, t), T q\left(x^{\prime}, t^{\prime}\right)\right), \\
2\left(\|x\|-t+\left\|x^{\prime}\right\|-t^{\prime}\right) & \geqslant d\left(T q(x, t), T q\left(x^{\prime}, t^{\prime}\right)\right) .
\end{aligned}
$$

Первое неравенство вытекает из неравенства треугольника и неравенства $t^{\prime} /\|x\| \leqslant 1$ :

$$
\begin{aligned}
3\left(\left\|x-x^{\prime}\right\|+\left|t-t^{\prime}\right|\right) \geqslant & \left\|x-x^{\prime}\right\|+2\left|t-t^{\prime}\right|+\frac{t^{\prime}}{\|x\|}\left\|x-x^{\prime}\right\|+\frac{t^{\prime}}{\|x\|}\left|\|x\|-\left\|x^{\prime}\right\|\right| \\
\geqslant & \left\|x-x^{\prime}\right\|+\left|t-t^{\prime}\right| \\
& \quad+\left\|\frac{t x}{\|x\|}-\frac{t^{\prime} x}{\|x\|}\right\|+\left\|\frac{t^{\prime} x}{\|x\|}-\frac{t^{\prime} x^{\prime}}{\|x\|}\right\|+\left\|\frac{t^{\prime} x^{\prime}}{\|x\|}-\frac{t^{\prime} x^{\prime}}{\left\|x^{\prime}\right\|}\right\| \\
\geqslant & \left\|x-x^{\prime}\right\|+\left\|\frac{t x}{\|x\|}-\frac{t^{\prime} x^{\prime}}{\left\|x^{\prime}\right\|}\right\|+\left|t-t^{\prime}\right| \\
\geqslant & \left\|\left(1-\frac{t}{\|x\|}\right) x-\left(1-\frac{t^{\prime}}{\left\|x^{\prime}\right\|}\right) x^{\prime}\right\|+\left|t-t^{\prime}\right| \\
= & d\left(T q(x, t), T q\left(x^{\prime}, t^{\prime}\right)\right) .
\end{aligned}
$$

Второе неравенство вытекает из неравенств $t^{\prime}-t \leqslant\|x\|-t$ и $t-t^{\prime} \leqslant\left\|x^{\prime}\right\|-t^{\prime}$ :

$$
\begin{aligned}
2\left(\|x\|-t+\left\|x^{\prime}\right\|-t^{\prime}\right) & =\|x\|-t+\left\|x^{\prime}\right\|-t^{\prime}+(\|x\|-t)+\left(\left\|x^{\prime}\right\|-t^{\prime}\right) \\
& \geqslant\|x\|-t+\left\|x^{\prime}\right\|-t^{\prime}+\left|t^{\prime}-t\right| \\
& =\left\|(\|x\|-t) \frac{x}{\|x\|}\right\|+\left\|\left(\left\|x^{\prime}\right\|-t^{\prime}\right) \frac{x^{\prime}}{\left\|x^{\prime}\right\|}\right\|+\left|t^{\prime}-t\right| \\
& \geqslant\left\|(\|x\|-t) \frac{x}{\|x\|}-\left(\left\|x^{\prime}\right\|-t^{\prime}\right) \frac{x^{\prime}}{\left\|x^{\prime}\right\|}\right\|+\left|t^{\prime}-t\right| \\
& =d\left(T q(x, t), T q\left(x^{\prime}, t^{\prime}\right)\right) .
\end{aligned}
$$

Во втором случае мы воспользуемся очевидным равенством

$$
\begin{aligned}
d_{C \mathbb{R}^{n}}\left(q(x, t), q\left(x^{\prime}, t^{\prime}\right)\right) \geqslant & \min \left\{d_{C \mathbb{R}^{n}}\left(q(x, t), q\left(z_{1}, t_{1}\right)\right)\right. \\
& \left.+d_{C \mathbb{R}^{n}}\left(q\left(z_{1}, t_{1}\right), q\left(z_{2}, t_{2}\right)\right)+d_{C \mathbb{R}^{n}}\left(q\left(z_{2}, t_{2}\right), q\left(x^{\prime}, t^{\prime}\right)\right)\right\},
\end{aligned}
$$


где минимум берется по всем точкам $\left(z_{1}, t_{1}\right),\left(z_{2}, t_{2}\right)$, лежашим в $i_{+}\left(\mathbb{R}^{n}\right)$. Заметим, что выражение в фигурных скобках равно

$$
\left|\|x\|-t_{1}\right|+\left|t-t_{1}\right|+\left|t_{1}-t_{2}\right|+||\left|x^{\prime}\right|\left|-t_{2}\right|+\left|t^{\prime}-t_{2}\right| .
$$

Тогда

$$
\begin{aligned}
& 2\left(\left|\|x\|-t_{1}\right|+\left|t-t_{1}\right|+\left|t_{1}-t_{2}\right|+\left|t_{2}-t^{\prime}\right|+\left|\left\|x^{\prime}\right\|-t_{2}\right|\right) \\
& \quad \geqslant\left|\|x\|-t_{1}\right|+\left|t_{1}-t_{2}\right|+\left|t_{2}-\left\|x^{\prime}\right\|\right|+\left|t-t^{\prime}\right| \\
& \quad \geqslant\left|\|x\|-\left\|x^{\prime}\right\|\right|+\left|t-t^{\prime}\right|=\left\|x^{\prime}\right\|-\|x\|+t^{\prime}-t \geqslant\|x\|-t+\left\|x^{\prime}\right\|-t^{\prime} .
\end{aligned}
$$

Последнее неравенство вытекает из $2 t^{\prime} \geqslant 2\|x\|$. Тогда

$$
\begin{aligned}
4 d_{C \mathbb{R}^{n}}\left(q(x, t), q\left(x^{\prime}, t^{\prime}\right)\right) & \geqslant\|x\|-t+\left\|x^{\prime}\right\|-t^{\prime}+\left|\|x\|-\left\|x^{\prime}\right\|\right|+\left|t-t^{\prime}\right| \\
& \geqslant\|x\|-t+\left\|x^{\prime}\right\|-t^{\prime}+\left|t-t^{\prime}\right|=d\left(T q(x, t), T q\left(x^{\prime}, t^{\prime}\right)\right) .
\end{aligned}
$$

4. Вероятностные меры. Вероятностные меры определяют функтор $P: \mathscr{A} \rightarrow \mathscr{A}$. Вероятностные меры с компактными носителями $P(X)$ на метрическом пространстве $(X, \rho)$ представляют собой метрическое пространство с метрикой КанторовичаРубинштейна

$$
\bar{\rho}\left(\mu_{1}, \mu_{2}\right)=\sup \left\{\left|\int f d \mu_{1}-\int f d \mu_{2}\right| \mid f \in S(X)\right\},
$$

где $S(X)$ означает пространство вешественнозначных сжимаюших функций. Заметим, что $X$ изометрично вкладьвается в $P(X)$ посредством дираковских мер $\delta_{x}$. Для любого $n$ определен подфунктор $P_{n}: \mathscr{A} \rightarrow \mathscr{A}$ вероятностных мер с носителями, состоящими не более чем из $n$ точек.

Для любых двух метрических пространств с отмеченными точками $X$ и $Y$ букет $X \vee Y$ определяется естественньм образом. В этом случае можно определить джойн $X * Y$ как подпространство $P_{2}(X \vee Y)$. Возникает естественный вопрос об изоморфизме конуса $C X$ и джойна $X * \mathbb{R}_{+}$.

Отметим также, что пространство $S(X)$, снабженное sup-нормой, содержит изометрическую копию $X$.

\section{§3. Абсолютные экстензоры}

Для любого объекта $X$ асимптотической категории $\mathscr{A}$ всякое замкнутое подмножество $Z \subset X$ представляет собой объект в $\mathscr{A}$. Здесь мы рассматриваем индуцированную метрику. Абсолютным әкстензором в категории $\mathscr{A}$ назьвается объект $Y$, обладающий свойством, что для всякого объекта $X$ и для любого его замкнутого подмножества $Z \subset X$ всякий морфизм $\varphi: Z \rightarrow Y$ продолжается до морфизма $\bar{\varphi}: X \rightarrow Y$. Возникает вопрос: что представляют из себя абсолютные экстензоры $A E(\mathscr{A})$ в категории $\mathscr{A}$ ? Аналогичный вопрос относится к $\tilde{\mathscr{A}}$. Одноточечное пространство не является абсолютным экстензором, так как неограниченные пространства не могут иметь совершенных отображений в точку.

Teopema 3.1. $\mathbb{R}_{+} \in A E(\mathscr{A}) u \mathbb{R}_{+} \in A E(\tilde{\mathscr{A}})$. 
ДокаЗАТЕльство. Сначала мы рассмотрим случай $\tilde{\mathscr{A}}$. Предположим, что $A \subset X$ замкнуто в $X$ и $\varphi: A \rightarrow \mathbb{R}_{+}-$морфизм с грубыми липшицевыми константами $\lambda$ и $s$. Пусть также $\|\varphi(x)\| \geqslant c\|x\|-b$, где $c \leqslant \lambda$. Естественно взять 0 в качестве базисной точки в $\mathbb{R}_{+}$. Мы построим не обязательно непрерывное продолжение $\varphi^{\prime}: X \rightarrow \mathbb{R}_{+}$с теми же константами $\lambda, s, c$ и $b$. Для этого воспользуемся трансфинитной индукцией (см. [17]). Занумеруем точки множества $X \backslash A$ трансфинитными числами. Предположим, что отображение $\varphi^{\prime}$ определено на множестве $B_{\alpha}=A \cup\left(\bigcup_{\beta<\alpha}\left\{x_{\beta}\right\}\right)$. Чтобы сохранить асимптотическую липшищевость отображения $\varphi^{\prime}$ с теми же константами $\lambda$ и $s$, нужно выбрать образ точки $x_{\alpha}$ в интервале $I_{x}=\left[\varphi^{\prime}(x)-\lambda d\left(x, x_{\alpha}\right)-s, \varphi^{\prime}(x)+\right.$ $\left.\left.\lambda d\left(x, x_{\alpha}\right)+s\right)\right]$ для всякого $x \in B_{\alpha}$. В предположении $\varphi^{\prime}\left(x^{\prime}\right) \leqslant \varphi^{\prime}(x)$ неравенство $\varphi^{\prime}(x)-\varphi^{\prime}\left(x^{\prime}\right) \leqslant \lambda d\left(x, x^{\prime}\right)+s$ вместе с неравенством треугольника для $x, x^{\prime}$ и $x_{\alpha}$ влекут, что

$$
\varphi^{\prime}(x)-\lambda d\left(x, x_{\alpha}\right)-s \leqslant \varphi^{\prime}\left(x^{\prime}\right)+\lambda d\left(x^{\prime}, x_{\alpha}\right)+s .
$$

Таким образом, $I_{x} \cap I_{x^{\prime}} \neq \varnothing$ для любых $x, x^{\prime} \in B_{\alpha}$. Для того чтобы сохранить неравенство $\left\|\varphi^{\prime}\left(x_{\alpha}\right)\right\|=\varphi^{\prime}\left(x_{\alpha}\right) \geqslant c\left\|x_{\alpha}\right\|-b$, мы должны выбрать образ точки $x_{\alpha}$ из луча $J_{\alpha}=\left[c\left\|x_{\alpha}\right\|-b, \infty\right)$. Заметим, что

$$
\begin{aligned}
\varphi^{\prime}(x)+\lambda d\left(x, x_{\alpha}\right)+s & \geqslant c\|x\|-b+\lambda d\left(x, x_{\alpha}\right)+s \\
& \geqslant c\left(\|x\|+d\left(x, x_{\alpha}\right)\right)-b+s \geqslant c\left\|x_{\alpha}\right\|-b+s \geqslant c\left\|x_{\alpha}\right\|-b .
\end{aligned}
$$

Следовательно, все интервалы $I_{x} \cap J_{\alpha}, x \in B_{\alpha}$, имеют попарно непустое пересечение. Теорема Хелли влечет, что пересечение $I=\left(\bigcap I_{x}\right) \cap J_{\alpha}$ непусто. Выберем $\varphi^{\prime}\left(x_{\alpha}\right) \in I$ произвольно. Согласно относительной версии предложения 1.3 сушествует непрерывное продолжение $\bar{\varphi}$ отображения $\varphi$, находящееся на конечном расстоянии от $\varphi^{\prime}$.

Мы не можем применить данное рассуждение в категории $\mathscr{A}$, так как при построении отображения $\bar{\varphi}$ мы не можем контролировать совершенность полученного отображения. Поэтому мы приводим другой аргумент.

Пусть $A \subset X$ замкнуто в $X$ и $\varphi: A \rightarrow \mathbb{R}_{+}$- совершенное асимптотически липшицево отображение с константами $\lambda$ и $s$. Возьмем $R$ из предложения 1.1 , примененного к $\varphi$ и $\alpha=2$. Положим $m=\lambda R+s$. Для любого $i$ определим $A_{i}=\varphi^{-1}([0, m i])$ и $B_{i}=A \backslash A_{i}$. Для любой положительной функции $\xi: E \rightarrow \mathbb{R}_{+}, E \subset X$, обозначим через $N_{\xi}(E)=\bigcup_{y \in E} B_{\xi(y)}(y) \xi$-окрестность множества $E$, где $B_{r}(y)$ означает замкнутый шар радиуса $r$ с центром в $y$. Если брать открытые шары, то получим открытую $\xi$-окрестность $O N_{\xi}(E)$ множества $E$. Определим последовательность функций $\xi_{i}$ по формуле $\xi_{i}(y)=\frac{1}{2} \lambda^{-1}(\varphi(y)-m i)$. Легко видеть, что $\xi_{i}>0$ на $B_{i+1}$. Обозначим $O_{i}=O N_{\xi_{i}}\left(B_{i+1}\right)$. По индукции мы построим семейство $\left\{C_{i}\right\}$ компактных подмножеств пространства $X$ со свойствами:

(1) $C_{i} \cap A=A_{i}$ и $\operatorname{Int}\left(C_{i}\right) \cap A=\operatorname{Int}_{A}\left(A_{i}\right)$

(2) $N_{\frac{m}{2 \lambda}}\left(C_{i}\right) \subset C_{i+1}$,

(3) $C_{i} \cap O_{i}=\varnothing$,

(4) $N_{\frac{m}{2 \lambda}}\left(C_{i}\right) \cap B_{i+1}=\varnothing$.

Условия (3) и (4) имеют чисто технический характер, они необходимы для гладкой работы индукции.

Положим $C_{0}=A_{0}$. Предположим, что множества $C_{0}, \ldots, C_{i}$ построены и удовлетворяют условиям (1)-(4). В силу совершенности $\varphi$ множество $A_{i+1}$ компактно. 
Возьмем компактное множество $C^{\prime} \subset X$, удовлетворяющее условию (1) для $i+1$. Заметим, что множество $C=C^{\prime} \cup N_{\frac{m}{2 \lambda}}\left(C_{i}\right)$ также удовлетворяет (1). Действительно, $C \cap A=\left(C^{\prime} \cap A\right) \cup\left(N_{\frac{m}{2 \lambda}}\left(C_{i}\right) \cap A\right)=A_{i+1} \cup\left(N_{\frac{m}{2 \lambda}}\left(C_{i}\right) \cap A_{i+1}\right) \cup\left(N_{\frac{m}{2 \lambda}}\left(C_{i}\right) \cap B_{i+1}\right)=A_{i+1}$ по условию (4). Определим $C_{i+1}=C \backslash O_{i+1}$. Чтобы проверить условие (1), достаточно показать, что $\mathrm{Cl}\left(O_{i+1}\right) \cap A_{i+1}=\varnothing$. Пусть $x \in \mathrm{Cl}\left(O_{i+1}\right) \cap A_{i+1}$, тогда найдется $y \in \mathrm{Cl}\left(B_{i+2}\right)$ c $d(x, y) \leqslant \xi_{i+1}(y)$. Так как $\varphi(x) \leqslant m(i+1)$, а $\varphi(y) \geqslant m(i+2)$, имеем $\varphi(y)-\varphi(x) \geqslant m$. Следовательно, $\lambda d(x, y)+s \geqslant m=\lambda R+s$, т.е. $d(x, y) \geqslant R$. Следовательно, $\varphi(y)-\varphi(x)<2 \lambda d(x, y)$. С другой стороны, $\varphi(y)-\varphi(x) \geqslant \varphi(y)-m(i+1)=$ $2 \lambda \xi_{i+1}(y) \geqslant 2 \lambda d(x, y)$. Противоречие.

Чтобы проверить условие (2), достаточно показать, что $N_{\frac{m}{2 \lambda}}\left(C_{i}\right) \cap O_{i+1}=\varnothing$. Предположим, что $x \in N_{\frac{m}{2 \lambda}}\left(C_{i}\right) \cap O_{i+1}$. Тогда найдутся точки $z \in C_{i}$ и $y \in B_{i+2}$ такие, что $d(x, z) \leqslant m /(2 \lambda)$ и $d(x, y)<\xi_{i+1}(y)$. Из неравенства треугольника вытекает:

$$
d(z, y)<\xi_{i+1}(y)+\frac{m}{2 \lambda}=\frac{1}{2 \lambda}(\varphi(y)-m i)=\xi_{i}(y)
$$

Следовательно, $z \in O_{i}$. Таким образом, $z \in C_{i} \cap O_{i}$, что противоречит условию (3).

Условие (3) для $C_{i+1}$ выполняется автоматически. Проверим условие (4). Предположим противное: пусть $x \in N_{\frac{m}{2 \lambda}}\left(C_{i+1}\right) \cap B_{i+2}$. Тогда найдется $y \in C_{i+1}$ с $d(x, y) \leqslant$ $m /(2 \lambda)$. С другой стороны, условие $(3)$ для $C_{i+1}$ влечет неравенство

$$
d(x, y) \geqslant \xi_{i+1}(x)=\frac{1}{2 \lambda}(\varphi(x)-m(i+1)) .
$$

Тогда $m(i+2) \geqslant \varphi(x)$, что означает, что $x \notin B_{i+2}$. Противоречие.

Заметим, что условие (2) влечет, что $\bigcup C_{i}=X$. Определим $\varphi\left(\partial C_{i}\right)=m i$ для всех $i$. В силу условия (1) мы получим непрерьвное продолжение отображения $\varphi$ на $A^{\prime}=A \cup\left(\bigcup_{i=0}^{\infty} \partial C_{i}\right)$. Рассмотрим множество $D_{i}=C_{i} \backslash \operatorname{Int}\left(C_{i-1}\right)$ и продолжим отображение $\left.\varphi\right|_{D_{i} \cap A^{\prime}}: D_{i} \cap A^{\prime} \rightarrow[m(i-1), m i]$ произвольно до непрерывного отображения $\bar{\varphi}_{i}: D_{i} \rightarrow[m(i-1), m i]$. Объединение $\bar{\varphi}=\bigcup \bar{\varphi}_{i}$ будет совершенньм непрерывным отображением $\bar{\varphi}: X \rightarrow \mathbb{R}_{+}$. Покажем, что $\bar{\varphi}$ асимптотически липшицево. Пусть $x, y \in X$ - две данные точки. Возьмем минимальное число $i$ такое, что $x \in C_{i}$. Аналогично возьмем $j$ для $y$. Не теряя обшности, можем предположить, что $j=i+k$. Тогда $|\bar{\varphi}(y)-\bar{\varphi}(x)| \leqslant m(k+1)$. По условию $(2)$ имеем: $d(x, y) \geqslant(k-1) m /(2 \lambda)$. Следовательно, $k+1 \leqslant(2 \lambda / m) d(x, y)+2$. Следовательно, $|\bar{\varphi}(y)-\bar{\varphi}(x)| \leqslant 2 \lambda d(x, y)+2 m$.

ЗАмечаниЕ 1 . Доказательство теоремы 3.1 в случае категории $\mathscr{A}$ меняет исходную константу Липшица $\lambda$ на $2 \lambda$. Не меняя доказательства, мы можем получить $\alpha \lambda$ для любого $\alpha>1$.

ЗАмечАниЕ 2 . Пространство $\mathbb{R}_{+}$не является абсолютным экстензором для морфизмов в смысле Ро. Например, если $X=\mathbb{R}_{+}$, а $A=\left\{n^{2} \mid n \in \mathbb{N}\right\} \subset \mathbb{R}_{+}$, то отображение $\varphi: A \rightarrow \mathbb{R}_{+}$, заданное формулой $\varphi\left(n^{2}\right)=2^{n}$, не имеет грубого продолжения в силу предложения 1.4 , так как никакое продолжение $\varphi$ не является липшищевым.

Пример 1. $\mathbb{R}^{n}$ не является $A E(\mathscr{A})$. Достаточно взять $X=\mathbb{R}^{n}, A=\bigcup S_{2^{n}}(0)-$ объединение сфер радиуса $2^{n}$ с центром в 0 , и определить $f: A \rightarrow \mathbb{R}^{n}$ как объединение отображений $f_{n}: S_{2^{n}}(0) \rightarrow S_{n}(0)$ степени $n$. Тогда у отображения $f$ нет совершенного продолжения.

Более простое доказательство состоит из замечания, что не сушествует совершенной ретракции $\mathbb{R}_{+}^{n+1}$ на $\mathbb{R}^{n}$. Это доказательство справедливо и для $\tilde{\mathscr{A}}$. 
Лемма 3.2. Полупространство $\mathbb{R}_{+}^{n}$ изоморфно в категории $\widetilde{\mathscr{A}}($ и, следовательно, в $\mathscr{A})$-й степени $\left(\mathbb{R}_{+}\right)^{n}$.

ДокАЗАТЕЛЬСтво. Имеется естественное вложение пространства $\left(\mathbb{R}_{+}\right)^{n}$ в $\mathbb{R}^{n}$, порожденное вложением $\mathbb{R}_{+} \subset \mathbb{R}$. Вложим $\mathbb{R}_{+}^{n}$ в $\mathbb{R}^{n}$ как полупространство, ограниченное гиперплоскостью $A: x_{1}+\cdots+x_{n}=0$ и содержашее $\left(\mathbb{R}_{+}\right)^{n}$. Определим отображение $T:\left(\mathbb{R}_{+}\right)^{n} \rightarrow \mathbb{R}_{+}^{n}$ следуюшим правилом: для любой прямой $l$, параллельной вектору $\bar{d}=(1, \ldots, 1)$, ограничение $T_{l}$ отображения $T$ на $l \cap\left(\mathbb{R}_{+}\right)^{n}$ есть параллельный перенос на вектор $\bar{w}_{l}$, параллельный $\bar{d}$, начальная точка которого есть $l \cap \partial\left(\mathbb{R}_{+}\right)^{n}$ и конечная $-l \cap A$. Таким образом, $T=\bigcup T_{l}$ является биекцией. Для любых двух точек $\bar{x}, \bar{y} \in\left(\mathbb{R}_{+}\right)^{n}$ имеем $\|T(\bar{x})-T(\bar{y})\|=\left\|\bar{x}+\bar{w}_{l}-\bar{y}-\bar{w}_{l^{\prime}}\right\|$, где $\bar{x} \in l, \bar{y} \in l^{\prime}, l\left\|l^{\prime}\right\| \bar{d}$. Тогда $\|T(\bar{x})-T(\bar{y})\| \leqslant\|\bar{x}-\bar{y}\|+\left\|\bar{w}_{l}-\bar{w}_{l^{\prime}}\right\|=\left|w_{l}-w_{l^{\prime}}\right|$, где $w_{l}$ обозначает длину вектора $\bar{w}_{l}$. Заметим, что $w_{l}=\frac{1}{n}\left(\sum x_{i}-m n\right)$, где $m=\min \left\{x_{i} \mid \bar{x}=\left(x_{1}, x_{2}, \ldots, x_{n}\right)\right\}$. Аналогично $w_{l^{\prime}}=\frac{1}{n}\left(\sum y_{i}-m^{\prime} n\right)$, где $m^{\prime}=\min \left\{y_{i}\right\}$. Тогда

$$
\begin{aligned}
\|T(\bar{x})-T(\bar{y})\| & =\frac{1}{n}\left|\sum x_{i}-\sum y_{i}+m^{\prime} n-m n\right|+\|\bar{x}-\bar{y}\| \\
& \leqslant \frac{1}{n}\left|\sum x_{i}-\sum y_{i}\right|+\left|m^{\prime}-m\right|+\|\bar{x}-\bar{y}\| .
\end{aligned}
$$

Не теряя обшности, можно предположить, что $m^{\prime}>m$. Предположим, что $m=x_{i}$, тогда $\left|m^{\prime}-m\right|=m^{\prime}-m \leqslant y_{i}-m=y_{i}-x_{i}=\left|y_{i}-x_{i}\right|$. Таким образом,

$$
\begin{aligned}
\|T(\bar{x})-T(\bar{y})\| & \leqslant\left(\frac{1}{n}+1\right) \sum\left|y_{i}-x_{i}\right|+\|\bar{x}-\bar{y}\| \\
& \leqslant(1+n) \frac{\sqrt{\sum\left|y_{i}-x_{i}\right|^{2}}}{\sqrt{n}}+\|\bar{x}-\bar{y}\| \\
& =\frac{1+n}{\sqrt{n}}\|\bar{x}-\bar{y}\|+\|\bar{x}-\bar{y}\| \leqslant n\|\bar{x}-\bar{y}\| .
\end{aligned}
$$

Та же липшицева константа годится и для $T^{-1}$. Заметим, что $\|T(\bar{x})\|=\left\|\bar{x}+\bar{w}_{l}\right\| \geqslant$ $\|x\|-w_{l} \geqslant\left(1-\frac{1}{\sqrt{2}}\right)\|\bar{x}\|$. Последнее неравенство вытекает из того, что $w_{l}=\left\|\operatorname{pr}_{A} \bar{x}\right\| \leqslant$ $\frac{1}{\sqrt{2}}\|\bar{x}\|$. Таким образом, норма $T$ отлична от нуля. Неравенство $\left\|T^{-1}(x)\right\| \geqslant\|x\|$ влечет, что и $T^{-1}$ имеет норму, отличную от нуля.

TeOpema 3.3. $\mathbb{R}_{+}^{n} \in A E(\mathscr{A}) u \mathbb{R}_{+}^{n} \in A E(\tilde{\mathscr{A}})$ для всех $n$.

ДокАЗАТЕльство. Согласно лемме 3.2 достаточно показать, что $\left(\mathbb{R}_{+}\right)^{n} \in A E(\mathscr{A})$. Рассмотрим базис $\bar{v}_{1}, \ldots, \bar{v}_{n}$ в $\mathbb{R}^{n}$, где $\bar{v}_{1}=(1, \varepsilon, \ldots, \varepsilon), \ldots, \bar{v}_{n}=(\varepsilon, \ldots, \varepsilon, 1)$ для достаточно малого $\varepsilon$. Определим проекцию $p_{i}:\left(\mathbb{R}_{+}\right)^{n} \rightarrow \mathbb{R}_{+}$на $i$-й сомножитель по формуле $p_{i}(\bar{z})=\bar{z} \cdot \bar{v}_{i}$. Геометрически $p_{i}$ является проекцией на ось $x_{i}$, параллельной плоскости $\alpha_{i}$, ортогональной к $\bar{v}_{i}$. Заметим, что $p_{i}$ является морфизмом в категории $\mathscr{A}$ в отличие от стандартной ортогональной проекции. Формула $p^{\prime}\left(\bar{e}_{i}\right)=\bar{v}_{i}$ определяет линейньй изоморфизм $p^{\prime}: \mathbb{R}^{n} \rightarrow \mathbb{R}^{n}$, где $\left\{\bar{e}_{i}\right\}$ - стандартньй базис. Легко проверить, что $p^{\prime}(\bar{z})=\sum p_{i}(\bar{z}) \bar{e}_{i}$. Обозначим через $p:\left(\mathbb{R}_{+}\right)^{n} \rightarrow p^{\prime}\left(\left(\mathbb{R}_{+}\right)^{n}\right)=V$ ограничение $p^{\prime}$ на $\left(\mathbb{R}_{+}\right)^{n}$. Обратное отображение $p^{-1}: V \rightarrow\left(\mathbb{R}_{+}\right)^{n}$ может быть продолжено до липшицева отображения $q:\left(\mathbb{R}_{+}\right)^{n} \rightarrow\left(\mathbb{R}_{+}\right)^{n}$. Чтобы это доказать, сначала заметим, что пространство $V$ представляет собой объединение лучей, выпушенных из 
начала координат и проходящих через симплекс $\sigma \subset \Delta^{n-1}$, полученньй из стандартного симплекса $\Delta^{n-1}=\left\{\bar{x} \mid \sum x_{i}=1, x_{i} \geqslant 0\right\}$ гомотетией $c: \Delta^{n-1} \rightarrow \sigma$ с центром в $\left(\frac{1}{n}, \ldots, \frac{1}{n}\right)$. Пусть $\gamma=c^{-1}: \sigma \rightarrow \Delta^{n-1}$ обозначает обратное отображение. Продолжим $\gamma$ до $\xi: \Delta^{n-1} \rightarrow \Delta^{n-1}$ с помощью радиального сжатия окрестности $\Delta^{n-1} \backslash \sigma$ в границу $\partial \Delta^{n-1}$. Отображение $\xi$ может быть линейно продолжено до отображения $\beta:\left(\mathbb{R}_{+}\right)^{n} \rightarrow\left(\mathbb{R}_{+}\right)^{n}$. Очевидно, что отображение $\beta$ липшицево. Ограничение $\left.\beta\right|_{V}$ на $V$ является линейным отображением, переводящим $\bar{v}_{i}$ в $(1+(n-1) \varepsilon) \bar{e}_{i}$. Следовательно, $p^{-1}=\left.\frac{1}{1+(n-1) \varepsilon} \beta\right|_{V}$. Положим $q=\frac{1}{1+(n-1) \varepsilon} \beta$.

Пусть $\varphi: A \rightarrow\left(\mathbb{R}_{+}\right)^{n}$ - морфизм в $\mathscr{A}(\widetilde{\mathscr{A}})$, а $A \subset Z$ - замкнутое подмножество. Тогда $p_{i}$ о $\varphi$-морфизм в $\mathscr{A}(\tilde{\mathscr{A}})$ для любого $i$. По теореме 3.1 сушествуют продолжения $\bar{\varphi}_{i}: Z \rightarrow \mathbb{R}_{+}$с константами $\lambda_{i}, s_{i}$. Рассмотрим отображение $\psi=q \circ \bar{\varphi}: Z \rightarrow\left(\mathbb{R}_{+}\right)^{n}$, где $\bar{\varphi}=\left(\bar{\varphi}_{1}, \ldots, \bar{\varphi}_{n}\right)$. Для любого $z \in A$ имеем

$$
\begin{aligned}
\psi(z) & =q\left(\bar{\varphi}_{1}(z), \ldots, \bar{\varphi}_{n}(z)\right)=q\left(p_{1} \varphi_{1}(z), \ldots, p_{n} \varphi_{n}(z)\right) \\
& =p^{-1}\left(p_{1} \varphi_{1}(z), \ldots, p_{n} \varphi_{n}(z)\right)=p^{-1} p \varphi(z)=\varphi(z) .
\end{aligned}
$$

Таким образом, $\psi$ является продолжением отображения $\varphi$. Отображение $\psi$ является совершенным (с ненулевой нормой в случае $\widetilde{\mathscr{A}}$ ), так как все отображения $\varphi_{i}$ совершенны (с ненулевой нормой). Отображение $\psi$ является асимптотически липшицевьм с константами $\lambda m$ и $s$, где $m=\max \left\{\lambda_{i}\right\}$, a $s=\max \left\{s_{i}\right\}$ и $\lambda$ - константа Липшица для $q$.

Подмножество $A \subset X$ метрического пространства $X$ назьвается собственным в макроскопическом смысле, если для любого $d$-окрестность $N_{d}(A)$ не совпадает с $X$ и $A$ неограничено.

ЛЕмма 3.4. Пусть $A$ - замкнутое собственное подмножество метрического пространства $(X, d)$, и пусть $g: A \rightarrow \mathbb{R}^{n}$ - асимптотически липиицево отображение. Тогда найдется такая окрестность $W \supset A$, что $\|g(a)\| \leqslant \lambda d(a, X \backslash W)+s$ для всех а $\in$ А при некоторых константах $\lambda u s$, допускающая асимптотически липиицево продолжение $\bar{g}: W \rightarrow \mathbb{R}^{n}$ отображсения $g$.

ДокАЗАтЕльство. По теореме 3.3 отображение $g: A \rightarrow \mathbb{R}^{n} \subset \mathbb{R}_{+}^{n+1}$ допускает асимптотически липшицево продолжение $g^{\prime}: X \rightarrow \mathbb{R}_{+}^{n+1}$ с константами $\lambda^{\prime}$ и $s^{\prime}$. Положим $\lambda=\sqrt{2} \lambda^{\prime}, s=\sqrt{2} s^{\prime}$ и $W=\left(g^{\prime}\right)^{-1}(V)$, где $V \subset \mathbb{R}_{+}^{n+1}$ - область под графиком функции $\|x\|$, определенной на $\mathbb{R}^{n}$. Тогда $\bar{g}: W \rightarrow \mathbb{R}^{n}$ есть композиция $g^{\prime}$ и ортогональной проекции $\pi: \mathbb{R}_{+}^{n+1} \rightarrow \mathbb{R}^{n}$. Для любых $a \in A$ и $w \in X \backslash W$ будет $\left\|g(a)-g^{\prime}(w)\right\| \geqslant \frac{1}{\sqrt{2}}\|g(a)\|$. Следовательно, $\lambda^{\prime} d(a, w)+s^{\prime} \geqslant \frac{1}{\sqrt{2}}\|g(a)\|$ и и тогда $\|g(a)\| \leqslant \lambda d(a, w)+s$. В силу произвольности выбора $w \in X \backslash W$ имеем $\|g(a)\| \leqslant \lambda d(a, X \backslash W)+s$ для любого $a \in A$.

ЗАмечАниЕ. Лемма 3.4 остается справедливой, если $\mathbb{R}^{n}$ заменить на $\mathbb{R}_{+}^{n}$.

Напомним, что отображение $f$ является грубо совершенным, если прообраз $f^{-1}(C)$ всякого ограниченного множества есть ограниченное множество.

ПРЕДЛОЖЕНИЕ 3.5. Для любого грубо совершенного (не обязательно непрерывного) отображсния $f: X \rightarrow \mathbb{R}_{+}$и любого совериенного метрического пространства $(X, d)$ существует асимптотически липиицева совершенная функиия $q: X \rightarrow \mathbb{R}_{+} c q \leqslant f$. 
ДоКАЗАТЕльСТВо. Обозначим $A_{k}=f^{-1}([0, k])$ и рассмотрим растущую последовательность конщентрических шаров $B_{m_{k}}\left(x_{0}\right)$ с целочисленными радиусами, $x_{0} \in X$, $k \in \mathbb{N}$, такую, что $A_{k} \subset B_{m_{k}}\left(x_{0}\right)$. Положим $q(x)=k-2+\frac{d\left(x, x_{0}\right)-m_{k-1}}{m_{k}-m_{k-1}}$ для $x \in B_{m_{k}}\left(x_{0}\right) \backslash B_{m_{k-1}}\left(x_{0}\right), k>1$. Положим $q\left(B_{m_{1}}\left(x_{0}\right)\right)=0$. Легко проверить, что $q$ - непрерьвная функция. Совершенность $q$ вытекает из того, что $\lim _{\|x\| \rightarrow \infty} q(x)=\infty$. Так как $f\left(B_{m_{k}}\left(x_{0}\right) \backslash B_{m_{k-1}}\left(x_{0}\right)\right) \geqslant k-1$, а $q\left(B_{m_{k}}\left(x_{0}\right) \backslash B_{m_{k-1}}\left(x_{0}\right)\right) \leqslant k-1$, получаем, что $q \leqslant f$. Выберем произвольные точки $x, y \in X$. Предположим, что $x \in B_{m_{k}}\left(x_{0}\right) \backslash B_{m_{k-1}}\left(x_{0}\right)$, а $y \in B_{m_{l}}\left(x_{0}\right) \backslash B_{m_{l-1}}\left(x_{0}\right)$, где $k \geqslant l$. Тогда

$|q(x)-q(y)|=\left|k-l+\frac{d\left(x, x_{0}\right)-m_{k-1}}{m_{k}-m_{k-1}}-\frac{d\left(y, x_{0}\right)-m_{l-1}}{m_{l}-m_{l-1}}\right| \leqslant|k-l|+2 \leqslant d(x, y)+3$.

Таким образом, отображение $q$ асимптотически липшищево.

Лемма 3.6. Предположим, что А есть замкнутое подмножсество в совершенном метрическом пространстве $(X, d)$, пусть $W \supset A$ обозначает замкнутую окрестность. Предполохсим, что $f: X \rightarrow \mathbb{R}_{+}-$грубо совершенное отображсение. Предположим, что заданы константы $\lambda, s$ и совериенное асимптотически липчичево отображсние $g: W \rightarrow \mathbb{R}$, ограниченное сверху, $g \leqslant f$, такие, что $\lambda d(a, X \backslash W)+s \geqslant g(a)$ для $a \in A$. Тогда существует совершенное асимптотически липчичево отображение $\bar{g}: X \rightarrow \mathbb{R}_{+}$такое, что $\bar{g} \leqslant f u$ $\left.\bar{g}\right|_{A}=g$.

ДоказАтельство. Так как $\mathbb{R}_{+} \in A E(\mathscr{A})$, сушествует совершенное асимптотически липшицево продолжение $g^{\prime}: X \rightarrow \mathbb{R}_{+}$отображения $g$. Предложение 3.5 , примененное к грубо совершенному отображению $f^{\prime}=\min \left\{f, g^{\prime}\right\}$, дает совершенное асимптотически липшицево отображение $q: X \rightarrow \mathbb{R}_{+}$такое, что $q \leqslant f$ на $X$ и $q \leqslant g$ на $W$. Определим $\varphi(x)=\frac{d(x, X \backslash W)}{d(x, A)+d(x, X \backslash W))}$ и $\bar{g}(x)=q(x)+\varphi(x)\left(g^{\prime}(x)-q(x)\right)$. Так как $q \leqslant \bar{g}$ и $\varphi(x)\left(g^{\prime}(x)-q(x)\right) \geqslant 0$, мы получаем, что $\bar{g}$ стремится к бесконечности при $\|x\| \rightarrow \infty$. Следовательно, $\bar{g}$ совершенно. Заметим, что $\left.\bar{g}\right|_{A}=g$. Покажем, что $\bar{g}$ асимптотически липшицево. Сначала заметим, что

$$
\begin{aligned}
|\varphi(x)-\varphi(y)|= & \frac{1}{d(y, A)+d(y, X \backslash W)} \frac{|d(y, A) d(x, X \backslash W)-d(x, A) d(y, X \backslash W)|}{d(x, A)+d(x, X \backslash W)} \\
= & \frac{1}{d(y, A)+d(y, X \backslash W)}|d(y, A) \varphi(x)-(1-\varphi(x)) d(y, X \backslash W)| \\
= & \frac{1}{d(y, A)+d(y, X \backslash W)} \mid d(y, A) \varphi(x)-d(x, A) \varphi(x) \\
& +(1-\varphi(x)) d(x, X \backslash W)-(1-\varphi(x)) d(y, X \backslash W) \mid \\
\leqslant & \frac{1}{d(y, A)+d(y, X \backslash W)}(|d(y, A)-d(x, A)| \varphi(x) \\
& +|d(x, X \backslash W)-d(y, X \backslash W)|(1-\varphi(x))) \\
\leqslant & \frac{1}{d(y, A)+d(y, X \backslash W)}(d(x, y) \varphi(x)+d(x, y)(1-\varphi(x))) \\
= & \frac{1}{d(y, A)+d(y, X \backslash W)} d(x, y) .
\end{aligned}
$$


Затем получим неравенство:

$$
\begin{aligned}
|\bar{g}(x)-\bar{g}(y)|= & \left|q(x)-q(y)+\varphi(x) g^{\prime}(x)-\varphi(x) q(x)-\varphi(y) g^{\prime}(y)+\varphi(y) q(y)\right| \\
\leqslant & |q(x)-q(y)|+\varphi(x)\left|g^{\prime}(x)-g^{\prime}(y)\right|+g^{\prime}(y)|\varphi(x)-\varphi(y)| \\
& +q(y)|\varphi(x)-\varphi(y)|+\varphi(x)|q(x)-q(y)| \\
\leqslant & 2|q(x)-q(y)|+\left|g^{\prime}(x)-g^{\prime}(y)\right|+2 g^{\prime}(y)|\varphi(x)-\varphi(y)| .
\end{aligned}
$$

Мы использовали неравенства $\varphi(x) \leqslant 1$ и $q(y) \leqslant g^{\prime}(y)$. Пусть $\lambda_{1}, s_{1}$ и $\lambda_{2}, s_{2}$ будут константы из определения асимптотической липшицевости для отображений $g^{\prime}$ и $q$. Тогда

$|\bar{g}(x)-\bar{g}(y)| \leqslant 2 \lambda_{2} d(x, y)+2 s_{2}+\lambda_{1} d(x, y)+s_{1}+2 g^{\prime}(y) \frac{1}{d(y, A)+d(y, X \backslash W)} d(x, y)$.

Пусть $\bar{\lambda}=\max \left\{\lambda, \lambda_{1}, \lambda_{2}\right\}$. Пусть $d(y, A)=d\left(y, y^{\prime}\right)$, где $y^{\prime} \in A$. Тогда

$$
\begin{aligned}
g^{\prime}(y) & \leqslant g\left(y^{\prime}\right)+\lambda_{1} d(y, A)+s_{1} \\
& \leqslant \lambda d\left(y^{\prime}, X \backslash W\right)+s+\lambda_{1} d(y, A)+s_{1} \\
& \leqslant \lambda d\left(y^{\prime}, y\right)+\lambda d(y, X \backslash W)+\lambda_{1} d(y, A)+s+s_{1} \\
& \leqslant 2 \bar{\lambda}(d(y, A)+d(y, X \backslash W))+s+s_{1} .
\end{aligned}
$$

Итак,

$$
\begin{aligned}
|\bar{g}(x)-\bar{g}(y)| & \leqslant 3 \bar{\lambda} d(x, y)+2 s_{2}+s_{1}+\frac{4 \bar{\lambda}(d(y, A)+d(y, X \backslash W))+2\left(s+s_{1}\right)}{d(y, A)+d(y, X \backslash W)} d(x, y) \\
& \leqslant 7 \bar{\lambda} d(x, y)+C
\end{aligned}
$$

при некоторой константе $C$.

\section{$\S$ 4. $A N E$, гомотопии, размерность}

Открытое множество $W \subset X$ в метрическом пространстве $(X, d)$ назовем асимптотически открытым или открытым в смысле категории $\mathscr{A}$, если $\sup \{d(x, X \backslash W) \mid x \in X\}=\infty$. Аналогично, открытым множеством в смысле $\tilde{\mathscr{A}}$ назовем открытое подмножество $W \subset X$ объекта $\widetilde{\mathscr{A}}$ со свойством, что найдется подмножество $A \subset W$ со свойством $d(x, X \backslash W) \geqslant k\|x\|$ при фиксированном $k$ для всех $x \in A$. В этом случае мы говорим, что $W$ является окрестностью множсества $A$ в смысле категории $\widetilde{\mathscr{A}}$. Множество $W$ является окрестностью собственного множества $A$ в смысле категории $\mathscr{A}(W$ - асимптотическая окрестность), если $d(x, X \backslash W)$ стремится к бесконечности как функция на $A$. Будем считать, что окрестность ограниченного множества есть окрестность в обычном смысле.

Ниже мы делаем попытку определить понятие абсолютного окрестностного экстензора в асимптотической категории $\mathscr{A}$ (или $\widetilde{\mathscr{A}})$. Так как у нас уже имеется понятие окрестности подмножества $A \subset X$, то наиболее естественным определением будет следующее

ОПРЕДЕЛЕНИЕ 1 (стандартное). Объект $Y \in \mathscr{A}$ (или $\widetilde{\mathscr{A}})$ называется абсолютным окрестностным әкстензором, $Y \in A N E(\mathscr{A})$, если для любого другого объекта $X$ 
и любого замкнутого подмножества $A \subset X$ всякий морфизм $f: A \rightarrow Y$ обладает продолжением $\bar{f}: W \rightarrow Y$ до морфизма замкнутой асимптотической окрестности.

Данное определение обладает следующим недостатком. Для него не выполняется стандартная теорема, связьваюшая $A N E$ и $A E: X \in A N E \Leftrightarrow C X \in A E$. Примером может служить парабола $X \in \mathbb{R}_{+}^{2}$, взятая с индуцированной метрикой. Тогда вложение $X$ в $C X$ не продолжается в категории $\mathscr{A}$ на $\mathbb{R}_{+}^{2}$. Если рассматривать геодезические метрики, то в качестве примера $X$ можно взять параболоид в $\mathbb{R}^{3}$, снабженный внутренней метрикой.

Заметим, что импликация $C X \in A E \Rightarrow X \in A N E$ вьполняется всегда.

ОПРЕДЕЛЕНИЕ 2 (категорное). $X \in A N E_{0}$, если $X \times \mathbb{R}_{+} \in A E$. Очевидно, что $A N E_{0}(\mathscr{A}) \subset A N E(\mathscr{A})$.

ПРЕДЛОЖЕНИЕ 4.1. $A N E_{0}(\widetilde{\mathscr{A}}) \subset A N E(\widetilde{\mathscr{A}})$.

ПрЕДЛОЖЕНИЕ 4.2. $\mathbb{R}^{n} \in A N E_{0}$ в обеих категориях $\mathscr{A}$ и $\tilde{\mathscr{A}}$.

ДокАЗАТЕЛЬСТво вытекает из теоремы 3.3.

Чтобы подчеркнуть аналогию с топологией, мы обозначим полуплоскость $\mathbb{R}_{+}^{2}$ чеpeз II.

ТЕОРЕма 4.3 (НЕТ - теорема о продолжении гомотопий). Предположсим, что $Y \in A N E(\widetilde{\mathscr{A}})$, и пусть $A$ - замкнутое подмножество в $X$. Тогда всякий морфизм $f: i_{0}(X) \cup A \widetilde{\times} \mathbb{I} \rightarrow Y$ продолжается до морфизма $\bar{f}: X \widetilde{\times} \mathbb{I} \rightarrow Y$.

ДокАЗАтЕльство. Так как $Y \in A N E$, существует продолжение $g: W \rightarrow Y$ отображения $f$ на окрестность $W$. Следовательно, $d(x,(X \widetilde{\times} \mathbb{I}) \backslash W) \geqslant \lambda\|x\|$ для некоторого $\lambda$. Выберем липшицево продолжение $\varphi: X \rightarrow \mathbb{R}_{+}$функции $\psi$, определенной на $A$ формулой $\psi(x)=d\left(x, x_{0}\right)+1$, так что область $D$ под графиком функции $\varphi$ лежит в $W$. Напомним, что $X \widetilde{\times} \mathbb{I}$ естественно вложено в $X \times \mathbb{R}_{+}$с $i_{0}(X)=X \times\{0\}$. Определим $h: X \widetilde{\times} \mathbb{I} \rightarrow D$ как отображение, переводящее всякий отрезок $\{x\} \times\left[0, d\left(x, x_{0}\right)+1\right]$ линейно в $\{x\} \times[0, \varphi(x)]$. Заметим, что $X \widetilde{\times} \mathbb{I}=\bigcup_{x \in X}\{x\} \times\left[0, d\left(x, x_{0}\right)+1\right]$. Пусть $\lambda^{\prime}$ будет константой Липшица для $\lambda$. Покажем, что отображение $h$ липшишево. Действительно,

$$
\begin{aligned}
d_{X} \times \mathbb{R}_{+} & \left(h(x, t), h\left(x^{\prime}, t^{\prime}\right)\right) \\
& =d_{X}\left(x, x^{\prime}\right)+\left|\frac{t}{\|x\|+1} \varphi(x)-\frac{t^{\prime}}{\left\|x^{\prime}\right\|+1} \varphi\left(x^{\prime}\right)\right| \\
& \leqslant d_{X}\left(x, x^{\prime}\right)+\frac{t}{\|x\|+1}\left|\varphi(x)-\varphi\left(x^{\prime}\right)\right|+\varphi\left(x^{\prime}\right)\left|\frac{t}{\|x\|+1}-\frac{t^{\prime}}{\left\|x^{\prime}\right\|+1}\right| \\
& \leqslant d_{X}\left(x, x^{\prime}\right)+\lambda^{\prime} d_{X}\left(x, x^{\prime}\right)+\frac{\varphi\left(x^{\prime}\right)}{\left\|x^{\prime}\right\|+1}\left(t-t^{\prime}\right)+\frac{t}{(\|x\|+1)\left(\left\|x^{\prime}\right\|+1\right)}\left|\left\|x^{\prime}\right\|-\|x\|\right| \\
& \leqslant\left(\lambda^{\prime}+1\right) d_{X}\left(x, x^{\prime}\right)+\left|t-t^{\prime}\right|+d_{X}\left(x, x^{\prime}\right) \leqslant\left(\lambda^{\prime}+2\right)\left(d_{X}\left(x, x^{\prime}\right)+\left|t-t^{\prime}\right|\right) \\
& =\left(\lambda^{\prime}+2\right) d_{X \times \mathbb{R}_{+}}\left((x, t),\left(x^{\prime}, t^{\prime}\right)\right) .
\end{aligned}
$$

Так как $\|x\|+1 \geqslant t$, имеем

$$
\|h(x, t)\|=\|x\|+\frac{t}{\|x\|+1} \varphi(x) \geqslant \frac{1}{2}(\|x\|+t)-\frac{1}{2}=\frac{1}{2}\|(x, t)\|-\frac{1}{2} .
$$


Таким образом, норма $h$ ненулевая, т.е. $h$ есть морфизм в $\mathscr{A}$. Определим $\bar{f}=g \circ h$.

ОПреДЕЛЕНИЕ. Гомотопия между двумя морфизмами $f, g: X \rightarrow Y$ есть морфизм $H: X \widetilde{\times} \mathbb{I} \rightarrow Y$ такой, что $\left.H\right|_{i_{-}(X)}=f$ и $\left.H\right|_{i_{+}(X)}=g$.

Гомотопия приводит к понятию гомотопической эквивалентности.

ПримеР. Как показано в [3], евклидово пространство $\mathbb{R}^{n}$ гомотопически эквивалентно гиперболическому пространству $\mathbb{H}^{n}$ в категории $\mathscr{A}$. Эти пространства не являются гомотопически эквивалентными в $\widetilde{\mathscr{A}}$, так как не сушествует липшищева отображения степени 1 пространства $\mathbb{R}^{n}$ на $\mathbb{H}^{n}$ с ненулевой нормой.

ОПРЕДЕЛЕНИЕ. МЫ говорим, что метрическое пространство имеет ограниченную геометрию, $X \in B G$, если для любого $L$ существует равномерно ограниченное покрытие $\mathscr{U}$ пространства $X$ с числом Лебега $>L$, обладающее конечным порядком.

Вышесказанное равносильно тому, что для любых $r$ и $\varepsilon$ существует $c(r, \varepsilon)$ такое, что $\varepsilon$-емкость всякого шара радиуса $r$ не превосходит $c(r, \varepsilon)$.

Для любого метрического пространства $X \in B G$ положим $d(L)=m(L)-1$, где $m(L)$ есть минимальньй порядок покрытия $\mathscr{U}$ из вышеприведенного определения.

ОПРЕДЕЛЕНИЕ 1 (Громов [1]). Супремум $\sup \left\{d(L) \mid L \in \mathbb{R}_{+}\right\}$называется асимnтотической размерностью пространства $X$ и обозначается как $\operatorname{as} \operatorname{dim} X$.

Приведенное определение является грубым аналогом (аналогом в смысле грубой категории) определения лебеговой размерности. В классической топологии имеется несколько различных определений размерности, которые дают один и тот же результат для достаточно хороших пространств, скажем, компактных метрических. Здесь мы рассмотрим макроаналоги определений Остранда, Александрова-Урысона и Александрова-Гуревича.

ОПРЕДЕЛЕНИЕ 2 (Громов [1]). $\operatorname{as} \operatorname{dim} X \leqslant n$, если для всякого $L>0$ найдутся $L$-дизъюнктные семейства $\mathscr{U}_{i}, i=0, \ldots, n$, равномерно ограниченных множеств такие, что $\bigcup_{i=0}^{n} \mathscr{U}_{i}$ является покрытием $X$.

Известно, что определения 1 и 2 согласуются между собой [1].

Пусть $\Delta$ - симплекс, лежаший в банаховом пространстве $(V,\|\cdot\|)$. Eмкостью $\Delta$ назовем расстояние от барицентра до границы $\partial \Delta$. Асимптотическим полиэдром $P$ назовем локально-конечньй полиэдр, наделенньй внутренней метрикой, ограничение которой на любой симплекс является метрикой, индуцированной из банахова пространства (одного для всех симплексов), такой, что емкость симплексов стремится к бесконечности при удалении от фиксированной базисной точки. Последнее означает, что для всякого $M>0$ найдется конечный подкомплекс $K \subset P$ такой, что емкость всякого симплекса из $P \backslash K$ больше чем $M$.

Предположим, что $X, Y$ - подпространства в $Z$, и пусть $\varphi: X \rightarrow Y$ - морфизм. По определению норма Александрова отображения $\varphi$ есть функция величины сдвига $\|\varphi\|_{A}: X \rightarrow \mathbb{R}_{+}$, где $\|\varphi\|_{A}(x)=d_{Z}(x, \varphi(x))$. Норма Александрова менњше заданной функции $f:\|\varphi\|_{A}<f$, если $\|\varphi\|_{A}(x)<f(x)$ для всех $x$ вне некоторого компактного множества. Мы говорим, что норма Урысона отображения $\varphi: X \rightarrow Y$ меньше функции $f,\|\varphi\|_{U}<f$, если для любого $R>0$ найдется компакт $C \subset X$ такой, что $\operatorname{diam}\left(\varphi^{-1}\left(B_{R}(\varphi(x))\right)\right)<f(x)$ для всех $x \in X \backslash C$. Заметим, что определение 
нормы Урысона не нуждается в объемлюшем пространстве. Известный подход Громова позволяет добиться того же и с нормой Александрова. Мы говорим, что норма Александрова-Громова отображения $\varphi: X \rightarrow Y$ не превосходит данной функции $f$, если существуют метрическое пространство $Z$ и изометрические вложения $X, Y \subset Z$ такие, что $\|\varphi\|_{A}<f$. В этом случае пишем $\|\varphi\|_{A G}<f$. Можно показать, что $(2+\varepsilon)\|\varphi\|_{A G}>\|\varphi\|_{U}$ для любого $\varepsilon>0$.

ОПРЕДЕЛЕниЕ 3. Метрическое пространство $X$ имеет размерность as $\operatorname{dim}_{*} X \leqslant n$, если для всякой совершенной функции $f: X \rightarrow \mathbb{R}_{+}$сушествует сжимаюшее отображение $\varphi: X \rightarrow K$ в $n$-мерньй асимптотический полиэдр такое, что $\|\varphi\|_{U}<f$.

Заметим, что в этом определении можно использовать норму Александрова-Громова.

Пусть $\mathscr{U}$ будет покрытием метрического пространства $X$, и пусть $x \in X$. Введем обозначения:

$$
\begin{aligned}
L_{\mathscr{U}}(x) & =\sup \{d(x, X \backslash U) \mid U \in \mathscr{U}\}, \\
\operatorname{mesh}_{\mathcal{U}}(x) & =\sup \{\operatorname{diam}(U) \mid U \in \mathscr{U}, x \in U\}, \\
\operatorname{order}_{\mathscr{U}}(x) & =m_{\mathscr{U}}(x)=\operatorname{card}\{U \in \mathscr{U} \mid x \in U\} .
\end{aligned}
$$

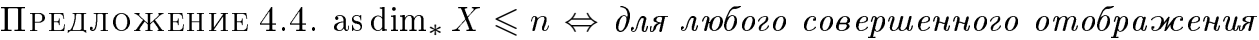
$f: X \rightarrow \mathbb{R}_{+}$существует равномерно ограниченное покрытие $\mathscr{U}$ порядка $\leqslant n+1$ $c \lim _{x \rightarrow \infty} L_{\mathscr{U}}(x)=\infty u c \operatorname{mesh}_{\mathcal{U}}(x)<f(x)$ для $x \in X \backslash C$, әде $C$ - компактное подмножество.

ДокАЗАТЕЛЬСТво. 1) Предположим, что as $\operatorname{dim}_{*} X=n$, и пусть дана функция $f: X \rightarrow \mathbb{R}_{+}$. По определению существует сжимающее отображение $\varphi: X \rightarrow K$ в асимптотический полиэдр $K$ размерности $n$ с $\|\varphi\|_{U}<f$. Не теряя обшности, можно считать, что $\varphi$ есть отображение на. Пусть $C_{R}$ обозначает компакт из определения неравенства $\|\varphi\|_{U}<f$. Рассмотрим фильтрацию $T_{1} \subset T_{2} \subset \cdots$ комплекса $K$ конечными подкомплексами такими, что $\varphi\left(C_{i}\right) \subset T_{i}$. Возьмем подразбиение $K^{\prime}$ комплекса $K$ такое, что mesh $_{K^{\prime}}(x) \leqslant i / 2$ для $x \in T_{i+1}$ и $K^{\prime}$ по-прежнему является асимптотическим полиэдром. Один из возможных путей построения такого подразбиения состоит из кубификации $K$ при помощи барицентрического разбиения, последующего регулярного подразбиения кубов и затем опять барицентрического подразбиения. При такой процедуре не теряется контроль за емкостью симплексов.

Определим покрытие $\mathscr{U}=\left\{\varphi^{-1}\left(\operatorname{OSt}\left(v, K^{\prime}\right)\right) \mid v \in\left(K^{\prime}\right)^{(0)}\right\}$, где $\operatorname{OSt}\left(v, K^{\prime}\right)$ означает открытую звезду вершины $v$ в $K^{\prime}$. Заметим, что

$$
\operatorname{diam} \varphi^{-1}\left(\operatorname{OSt}\left(v, K^{\prime}\right)\right) \leqslant \operatorname{diam} \varphi^{-1}\left(B_{i}(y)\right)<f(x)
$$

для всех $y \in \operatorname{OSt}\left(v, K^{\prime}\right)$ и всех $x \in \varphi^{-1}(y)$, где $v \in T_{i+1} \backslash T_{i}$. Таким образом, $\operatorname{mesh}_{\mathcal{U}}(x)<f(x)$ для $x \in X \backslash C_{1}$.

2) Пусть дана функция $f: X \rightarrow \mathbb{R}_{+}$. Можем предполагать, что $f(x) \leqslant\|x\|=$ $d_{X}\left(x, x_{0}\right)$. Положим $g(t)=\inf \left\{f(x) \mid x \in X \backslash B_{t}\left(x_{0}\right)\right\}$. Определим

$$
\bar{f}(x)=\frac{1}{4} g(\|x\|-f(x))
$$


Заметим, что $\lim _{\|x\| \rightarrow \infty} \bar{f}(x)=\infty$. Рассмотрим равномерно ограниченное покрытие

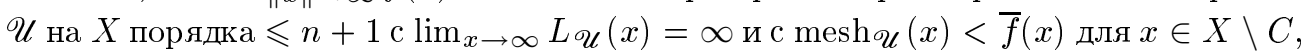
где $C$ - фиксированный компакт. Пусть $\varphi: X \rightarrow N(\mathscr{U})$ означает проекцию в нерв покрытия $\mathscr{U}$. Существует кусочно-евклидова метрика на $N(\mathscr{U})$ такая, что $N(\mathscr{U})-$ асимптотический полиэдр и отображение $\varphi$ - сжимающее отображение. Нам нужно показать, что для любого $R$ для $x$, близких к бесконечности, вьполняется неравенство $\operatorname{diam}\left(\varphi^{-1}\left(B_{R}(\varphi(x))\right)\right)<f(x)$. Сначала заметим, что для $x$, близких к бесконечности, шар $B_{R}(\varphi(x))$ содержится в звезде $\operatorname{St}(\{V\}, N(\mathscr{U}))$, где $x \in V \in \mathscr{U}$. Следовательно,

$$
\varphi^{-1}\left(B_{R}(\varphi(x))\right) \subset \bigcup_{\substack{n \in \neq \neq \\ U \in \mathscr{U}}} U=V^{*} .
$$

Пусть $y \in U$ и $U \cap V \neq \varnothing, U \in \mathscr{U}$. Тогда

$$
\begin{aligned}
d(x, y) & \leqslant \operatorname{mesh}_{\mathcal{U}}(x)+\operatorname{mesh}_{\mathcal{U}}(y)<\bar{f}(x)+\bar{f}(y) \\
& =\frac{1}{4}(g(\|x\|-f(x))+g(\|y\|-f(y))) \\
& \leqslant \frac{1}{4}\left(f(x)+\inf \left\{f(z) \mid z \in X \backslash B_{\|y\|-f(y)}\left(x_{0}\right)\right\}\right) .
\end{aligned}
$$

В силу неравенства треугольника имеем $\|y\|-f(y) \leqslant\|x\|$. Следовательно, $x \in$ $B_{\|y\|-f(y)}\left(x_{0}\right)$ и, значит,

$$
\inf \left\{f(z) \mid z \in X \backslash B_{\|y\|-f(y)}\left(x_{0}\right)\right\} \leqslant f(x) .
$$

Итак, $d(x, y)<\frac{1}{2} f(x)$. Следовательно, $\operatorname{diam}\left(V^{*}\right)<f(x)$.

Метод доказательства теоремы 1.1 из [18] позволяет доказать

Предложение 4.5. $\operatorname{as} \operatorname{dim}_{*} X \leqslant \operatorname{as} \operatorname{dim} X$ для всеx $X$.

Следуюшее определение макроскопической размерности основано на идее Александрова-Гуревича, используюшей продолжение отображений в сферу.

ОПРЕДЕЛЕНИЕ $4 . \operatorname{dim}^{c} X \leqslant n$, если для любого замкнутого подмножества $A \subset X$ и всякого морфизма $f: A \rightarrow \mathbb{R}^{n+1}$ в $\mathscr{A}$ сушествует расшширение $\bar{f}: X \rightarrow \mathbb{R}^{n+1}$.

Заметим, что $\mathbb{R}^{n+1}$ служит аналогом $n$-сферы в категории $\mathscr{A}$. Аналогия отражается тем фактом, что $\mathbb{R}^{n+1}$ является $n$-кратной надстройкой в $\mathscr{A}$ (см. лемму 2.4 ) над прямой $\mathbb{R} \simeq \mathbb{R}_{+} \Perp \mathbb{R}_{+}$, которая изоморфна дизъюнктной сумме двух полупрямых. Полупрямая $\mathbb{R}_{+}$является аналогом точки, так как является минимальным абсолютным экстензором. Таким образом, вешественная прямая $\mathbb{R}$ в категории $\mathscr{A}$ есть объединение двух “точек", т.е. является 0- “сферой”.

B $\S 6$ мы докажем, что грубая размерность $\operatorname{dim}^{c} X$ не превосходит $\operatorname{as} \operatorname{dim}_{*} X$.

ОПРЕДЕЛЕНИЕ. Метрическое пространство $X$ имеет слабо растущую размерность, если $\lim _{L \rightarrow \infty} \frac{d(L)}{L}=0$. 


\section{$\S$ 5. Аппроксимация полиэдрами, когомологии и размерность}

Всякое совершенное метрическое пространство ограниченной геометрии допускает античеховскую аппроксимацию полиэдрами. По определению это прямая последовательность $\left\{K_{i}, g_{i+1}^{i}: K_{i} \rightarrow K_{i+1}\right\}$ симплищиальных комплексов с симплищиальными проекциями $g_{i+1}^{i}$ вместе с сжимаюшими отображениями $f_{i}: X \rightarrow K_{i}$ такими, что $f_{i+1}$ гомотопно $g_{i+1}^{i} \circ f_{i}$. Метрика на $K_{i}$ такова, что все симплексы изоморфны стандартным с длиной ребра $L_{i}$ и $\lim _{i \rightarrow \infty} L_{i}=\infty$. Более того, существуют сжимающие отображения $f_{i+1}^{i}: K_{i} \rightarrow K_{i+1}$ такие, что $f_{i+1}=f_{i+1}^{i} \circ f_{i}$, а $g_{i+1}^{i}$ являются симплициальными аппроксимациями отображений $f_{i+1}^{i}$. В случае когда $X$ равномерно стягиваемо, у отображений $f_{i}$ имеются левые совершенно гомотопически обратные отображения $p_{i}: K_{i} \rightarrow X$ такие, что $f_{i+1}^{i}$ и $f_{i+1}$ ○ $p_{i}$ гомотопны посредством совершенных гомотопий (см. [2], [19] для дальнейших деталей).

Мы назовем симплициальные комплексы, участвуюшие в определении античеховской аппроксимации, равномерными полиэдрами. Число $L$ будем называть мелкостью равномерного полиэдра $K$ и обозначать $L=\operatorname{mesh}(K)$.

Следуя Дж. Ро [2], [19], мы определим античеховские гомологии (грубые гомологии) пространства $X$ как $\widehat{H}_{*}(X)=\lim _{\longrightarrow}\left\{H_{*}\left(K_{i}\right)\right\}$. Если рассматривать гомологии с бесконечными локально конечными цепями, то получатся экзотические гомологии, обозначенные Ро как $H X_{*}(X)=\underset{\lim }{\longrightarrow}\left\{H_{*}^{l f}\left(K_{i}\right)\right\}$.

Напомним, что в классической топологии компактных метрических пространств имеется одна естественная теория когомологий (все разумные теории совпадают) и две теории гомологий: Чеха и Стинрода. Обе теории гомологий необходимы. Теория гомологий Чеха не точна, но зато непрерьвна. Теория гомологий Стинрода точна, но не является непрерьвной. Похожая ситуация сложилась и в макромире, только там возникают проблемы с когомологиями. Античеховские когомологии могут быть определены аналогично $\widehat{H}^{*}(X)=\lim \left\{H^{*}\left(K_{i}\right)\right\}$, а также для компактных носителей $\widehat{H}_{c}^{*}(X)=\lim _{\longleftarrow}\left\{H_{c}^{*}\left(K_{i}\right)\right\}$. Напомним, что гомологии Стинрода компактного метрического пространства $Y$ определяются по обратной последовательности из полиэдров, $Y=\lim \left\{L_{i}, q_{i}^{i+1}\right\}$, следуюшим образом: пусть $T$ - телескоп системы $\left\{L_{i}, q_{i}^{i+1}\right\}$, начинаюшийся с цилиндра постоянного отображения $q_{0}^{\prime}: L_{1} \rightarrow \mathrm{pt}$, тогда по определению $H_{k}^{s}(Y)=H_{k+1}^{l f}(T)$. В случае асимптотической категории мы также рассмотрим телескоп $T$ прямой системы $\left\{K_{i}, g_{i+1}^{i}\right\}$, задаюшей античеховскую аппроксимацию пространства $X$. Тогда когомологии Ро определяются как $H_{R}^{k}(X)=H^{k}(T)$. Чтобы определить грубые когомологии Ро с компактными носителями, рассмотрим телескоп $T^{\alpha}$ одноточечных компактификаций $\left\{\alpha K_{i}, \alpha g_{i+1}^{i}\right\}$. Положим $H_{R, c}^{k}(X)=H^{k}\left(T^{\alpha}\right)$. В обозначениях Ро $H_{R, c}^{k}(X)=H X^{k}(X)$. Параллельно случаю гомологий Стинрода для компактов существует короткая точная последовательность, связывающая когомологии Ро с античеховскими [2]

$$
0 \rightarrow \lim ^{1} H_{c}^{k-1}\left(K_{i}\right) \rightarrow H_{R, c}^{k}(X) \rightarrow \widehat{H}_{c}^{k}(X) \rightarrow 0 .
$$

Пусть $A \subset X$ - собственное замкнутое подмножество (в смысле категории $\mathscr{A}$ ). Тогда пара $(X, A)$ обладает античеховской аппроксимацией $\left\{\left(K_{i}, L_{i}\right), g_{i+1}^{i}\right\}$, что позволяет определить грубые гомологии и когомологии пары $(X, A): \widehat{H}_{i}^{l f}(X, A)$, $\widehat{H}_{c}^{i}(X, A)$ и $\widehat{H}_{R, c}^{i}(X, A)$. 
ПРЕДЛОЖЕНИЕ 5.1. Для любого замкнутого подмножества $A \subset X$ существулот точные последовательности пары $(X, A)$ :

$$
\begin{aligned}
& \text { 1) } \cdots \longrightarrow \widehat{H}_{i}^{l f}(A) \longrightarrow \widehat{H}_{i}^{l f}(X) \longrightarrow \widehat{H}_{i}^{l f}(X, A) \longrightarrow \widehat{H}_{i-1}^{l f}(A) \longrightarrow \cdots, \\
& \text { 2) } \cdots \longleftarrow H_{R, c}^{i}(A) \longleftarrow H_{R, c}^{i}(X) \longleftarrow H_{R, c}^{i}(X, A) \longleftarrow H_{R, c}^{i-1}(A) \longleftarrow \cdots .
\end{aligned}
$$

ДокАЗАТЕЛЬСтво. Заметим, что

$$
\widehat{H}_{k}^{l f}(X)=\underline{\lim }_{k} H_{k}^{l f}\left(K_{i}\right)=\lim _{\longrightarrow} H_{k}^{s}\left(\alpha K_{i}\right)=\lim _{\longrightarrow} H_{k}^{s}\left(T_{i}^{\alpha}\right)=H_{k}^{s}\left(T^{\alpha}\right),
$$

где $T_{i}^{\alpha}$ - телескоп длины $i$ системы $\left\{K_{j}, g_{j+1}^{j}\right\}_{j<i}$. В таком случае требуемые точные последовательности пары вытекают из точных последовательностей пар $\left(T_{X}^{\alpha}, T_{A}^{\alpha}\right)$.

Включение $\alpha X$ в телескоп $\alpha T_{X}$ индуцирует гомоморфизмы $c_{*}: H_{*}^{l f}(X) \rightarrow \widehat{H}_{*}^{l f}(X)$ и $c^{*}: H_{R, c}^{*}(X) \rightarrow H_{c}^{*}(X)$.

Теорема 5.2 [2], [19]. Для любого равномерно стягиваемого пространства $X$ гомоморфизмы $c_{*}$ и $c^{*}$ являются изоморфизмами.

Теорема 5.3. Пусть $A$ - замкнутое подмнохсество равномерно стягиваемого пространства $X$. Тогда имеются равенство

1) $\widehat{H}_{*}^{l f}(X, A)=\underset{k}{\lim } H_{*}^{l f}\left(X, N_{k}(A)\right)$

и точная последовательность

2) $0 \rightarrow \overleftarrow{\lim }^{1} H_{c}^{*-1}\left(X, N_{k}(A)\right) \rightarrow H_{R, c}^{*}(X, A) \rightarrow \underset{k}{\lim } H_{c}^{*}\left(X, N_{k}(A)\right) \rightarrow 0$, где $N_{k}(A)$ - замкнутая $k$-окрестность множества $A$.

ДокаЗАТЕльство. Пусть $j_{k+1}^{k}: N_{k}(A) \rightarrow N_{k+1}(A)$ обозначает включение. Обозначим через $S^{\alpha}$ телескоп, порожденньй одноточечными компактификациями $\alpha N_{k}(A)$ и их отображениями $\alpha j_{k+1}^{k}$. Так как $\alpha j_{k+1}^{k}$ суть вложения, сушествует естественное вложение $S^{\alpha} \subset \alpha X \times \mathbb{R}_{+}$. Покажем, что пара $\left(\alpha X \times \mathbb{R}_{+}, S^{\alpha}\right)$ совершенно гомотопически эквивалентна паре $\left(T_{X}^{\alpha}, T_{A}^{\alpha}\right)$. Можем предполагать, что античеховская аппроксимация пространства $X$ выбрана так, что $f_{i}\left(N_{i}(A)\right) \subset L_{i}$. Определим отображение $f: X \times \mathbb{R}_{+} \rightarrow T_{X}$ как объединение $\bigcup f_{i}$ на множестве $X \times \mathbb{N}$, продолженное на все $X \times \mathbb{R}_{+}$с помощью гомотопий между $f_{i+1}^{i}$ и $g_{i+1}^{i}$. Для любого $i$ найдется $k(i)$ такое, что $p_{i}\left(L_{i}\right) \subset N_{k(i)}(A)$ и $\left.p_{i} \circ f_{i}\right|_{N_{k(i-1)}(A)}$ гомотопно тождественному отображению $\operatorname{id}_{N_{k(i-1)}(A)}$ в пространстве $N_{k(i)}(A)$. Определим отображение $p: T_{X} \rightarrow X \times \mathbb{R}_{+}$ как продолжение объединения отображений $p_{i}: K_{i} \rightarrow X \times k(i)$. Несложная проверка показьвает, что $f$ и $p$ определяют совершенную гомотопическую эквивалентность пар $\left(X \times \mathbb{R}_{+}, S\right)$ и $\left(T_{X}, T_{A}\right)$. Следовательно, эти отображения индуцируют совершенную гомотопическую эквивалентность пар $\left(T_{X}^{\alpha}, T_{A}^{\alpha}\right)$ и $\left(\alpha X \times \mathbb{R}_{+}, S^{\alpha}\right)$. Тогда $\widehat{H}_{i}^{l f}(X, A)=H_{i}^{s}\left(T_{X}^{\alpha}, T_{A}^{\alpha}\right)=H_{i}^{s}\left(\alpha X \times \mathbb{R}_{+}, S^{\alpha}\right)=\lim _{\longrightarrow} H_{i}^{l f}\left(X, N_{k}(A)\right)$ и $H_{R, c}^{*}(X, A)=H^{*}\left(T_{X}^{\alpha}, T_{A}^{\alpha}\right)=H^{*}\left(\alpha X \times \mathbb{R}_{+}, S^{\alpha}\right)$.

Точная последовательность Милнора для $H^{*}\left(\alpha X \times \mathbb{R}_{+}, S^{\alpha}\right)$ может быть записана KaK

$$
0 \rightarrow{\underset{k}{\lim ^{1}}}^{1} H_{c}^{*-1}\left(X, N_{k}(A)\right) \rightarrow H_{R, c}^{*}(X, A) \rightarrow \underset{k}{\lim _{k}} H_{c}^{*}\left(X, N_{k}(A)\right) \rightarrow 0
$$


Вышеприведеншые определения античеховских гомологий, а также когомологий Ро и античеховских когомологий легко переносятся на обобщенные гомологии и когомологии: по определению $\widehat{M}_{*}^{l f}(X, A)=M_{*}^{s}\left(T_{X}^{\alpha}, T_{A}^{\alpha}\right), M_{R, c}^{*}(X, A)=M^{*}\left(T_{X}^{\alpha}, T_{A}^{\alpha}\right)$, и $\widehat{M}^{*}(X, A)=\lim M^{*}\left(K_{i}, L_{i}\right)$. Утверждения 5.1-5.3 также выполняются и для обобшенных (ко)гомологий.

ОПРЕДЕЛЕНИЕ. Асимптотической когомологической размерностью совершенного метрического пространства $X$ с ограниченной геометрией $(X \in B G)$ с группой коэффициентов $G$ назьвается число

$$
\operatorname{asim}_{G} X=\sup \left\{n \mid H_{R, c}^{n}(X, A ; G) \neq 0, A \subset_{\mathrm{Cl}} X\right\} .
$$

ТЕОрема 5.4. Для любого равномерно стягиваемого пространства $X$, с $X \in$ $B G$, выполняются неравенства:

$$
\operatorname{as} \operatorname{dim}_{G} X \leqslant \operatorname{dim}_{G} X \leqslant \operatorname{dim} X \leqslant \operatorname{as} \operatorname{dim} X,
$$

где $\operatorname{dim}_{G} X u \operatorname{dim} X-$ объчная когомологическая и лебегова размерности.

ДоКАЗАТЕЛЬСТВо вытекает из теоремы 5.3 и раздела $1 . \mathrm{F}_{1}$ книги [1].

Лемма Громова (лемма 6.1) влечет следуюшую теорему.

ТеОрема 5.5. Пространство с ограниченной геометрией X имеет размерность аs $\operatorname{dim} X \leqslant n$ тогда и только тогда, когда $X$ допускает античеховскую аппроксимацию п-мернымми симплищиальнымми комплексами.

\section{$\S$ 6. Корона Хигсона}

Пусть $f: X \rightarrow \mathbb{R}$ - непрерывная функция, заданная на метрическом пространстве $X$, и пусть $B_{R}(x)$ означает шар радиуса $R$ с центром в $x . R$-вариацией $\operatorname{Var}_{R} f(x)$ функции $f$ в точке $x \in X$ назьвается число, равное $\sup _{y \in B_{R}(x)}|f(x)-f(y)|$. Обозначим через $C_{h}(X)$ семейство ограниченных непрерывных функций на $X$, обладаюших свойством, что $\lim _{x \rightarrow \infty} \operatorname{Var}_{R} f(x)=0$ для любого фиксированного $R$. Компактификацией Хигсона метрического пространства $X$ является замыкание $X$, вложенного в $\mathbb{R}^{C_{h}(X)}$ посредством $\prod_{f \in C_{h}(X)} f$. Нарост компактификации $\nu X=\bar{X} \backslash X$ назьвается короной Хигсона. Заметим, что корона Хигсона является ковариантным функтором $\nu: \mathscr{C} \rightarrow$ Comp, действуюшим из грубой категории в категорию бикомпактов (компактных хаусдорфовых пространств) [2].

Напомним, что кусочно-евклидов симплициальный комплекс $K$ имеет мелкость $D$, $\operatorname{mesh}(K)=D$, если все симплексы изометричны стандартным евклидовым с длиной стороны $D$ и метрика на $K$ - геодезическая метрика, индуцированная метрикой на симплексах. Отображение между метрическими пространствами $f: X \rightarrow Y$ называется сәсимающим, если $d_{Y}\left(f(x), f\left(x^{\prime}\right)\right) \leqslant d_{X}\left(x, x^{\prime}\right)$ для любых $x, x^{\prime} \in X$. Отображение $f: X \rightarrow Y$ называется равномерно коограниченным, если для любого $R>0$ найдется константа $C$ такая, что диаметр прообразов $f^{-1}\left(B_{R}(y)\right)$ не превосходит $C$.

Лемма 6.1 [1]. Для совериенного метрического пространства $X$ следующие условия әквивалентны:

(1) $\operatorname{asdim} X \leqslant n$; 
(2) для любого $D>0$ существует равномерно коограниченное сжимающее отображение $f: X \rightarrow K$ в $n$-мерный симплициальный комплекс размерности $n$.

Teopema 6.2. Ecлu as $\operatorname{dim} X<\infty, m o$ as $\operatorname{dim} X=\operatorname{dim} \nu X$.

ДоказАтельство. Неравенство as $\operatorname{dim} X \geqslant \operatorname{dim} \nu X$ доказано в [18].

Пусть $\operatorname{as} \operatorname{dim} X=m$, покажем, что $\operatorname{dim} \nu X \geqslant m$. В силу леммы 6.1 сушествует последовательность сжимающих отображений $\varphi_{i}: X \rightarrow K_{i}$ c $\operatorname{dim} K_{i}=m$ и $D_{i}=$ $\operatorname{mesh}\left(K_{i}\right) \rightarrow \infty$. Пусть $S=\{f: X \rightarrow Y\}$ будет семейство отображений, определим константу Липшица $L(S)$ семейства $S$ как inf $f \in S\left\{\lambda \mid d_{Y}\left(f(x), f\left(x^{\prime}\right)\right) \leqslant \lambda_{X}\left(x, x^{\prime}\right)\right\}$. Если число $L(S)$ не определено, то полагаем $L(S)=\infty$.

Пусть $g: X \rightarrow B^{n}$ - несущественное отображение, т.е. отображение, допускающее выметание $q: X \rightarrow \partial B^{n}$ с $\left.q\right|_{g^{-1}\left(\partial B^{n}\right)}=\left.g\right|_{g^{-1}\left(\partial B^{n}\right)}$. Обозначим через $S(g)$ множество всех непрерывных выметаний отображения $g$. Фиксируем базисную точку $x_{0} \in X$. Определим

$$
\lambda_{i}^{r}=\sup \left\{L\left(S\left(\left.\varphi_{i}\right|_{\varphi_{i}^{-1}\left(\Delta^{m}\right)}\right)\right) \mid \Delta^{m} \subset K_{i} \backslash \operatorname{Int} B_{r}\left(\varphi_{i}\left(x_{0}\right)\right)\right\}
$$

Положим $\lambda_{i}=\varlimsup_{r \rightarrow \infty} \lambda_{i}^{r}$. Покажем, что последовательность $\frac{D_{i}}{\lambda_{i}}$ ограничена сверху. Предположим противное, тогда найдется последовательность $i_{k} \mathrm{c} \frac{D_{i_{k}}}{\lambda_{i_{k}}}>k$. Следовательно, $\frac{D_{i_{k}}}{\lambda_{i_{k}}^{r_{k}}}>i$ для некоторых $r_{k}$. Тогда $\lambda_{i_{k}}^{r_{k}}<\infty$ и, следовательно, отображения $\left.\varphi_{i_{k}}\right|_{\varphi_{i_{k}}^{-1}\left(\Delta^{m}\right)}: \varphi_{i_{k}}^{-1}\left(\Delta^{m}\right) \rightarrow \Delta^{m}$ являются несущественными для любых $m$-симплексов $\Delta^{m} \subset K_{i_{k}} \backslash \operatorname{Int} B_{r_{k}}\left(\varphi_{i_{k}}\left(x_{0}\right)\right)$. Для любого такого симплекса $\Delta^{m}$ рассмотрим вьметание $\psi_{\Delta^{m}}: \varphi_{i_{k}}^{-1^{k}}\left(\Delta^{m}\right) \rightarrow \partial \Delta^{m}$ с константой Липшица, равной $L\left(S\left(\left.\varphi_{i_{k}}\right|_{\varphi_{i_{k}}^{-1}\left(\Delta^{m}\right)}\right)\right)$. Объединение этих выметаний определяет равномерно коограниченное отображение $\psi_{k}: X \rightarrow K_{i_{k}}^{(m-1)} \cup L_{k}$, где $L_{k}-$ звездная окрестность множества $B_{r_{k}}\left(\varphi_{i_{k}}\left(x_{0}\right)\right)$. Рассмотрим симплициальное отображение, порожденное сжатием $L_{k}$ в точку: $p: K_{i_{k}}^{(m-1)} \cup L_{k} \rightarrow M_{k}$. Тогда $\operatorname{dim} M_{k}=m-1$. Более того, отображение $p$ - сжимающее относительно кусочно-евклидовой метрики на $M_{k}$ с $\operatorname{mesh}\left(M_{k}\right)=D_{i_{k}}$. Если мы умножим метрику на $M_{k}$ на число $\frac{1}{\lambda_{i_{k}}^{r_{k}}}$, то композищия $p \circ \psi_{k}$ будет сжимаюшим отображением. Заметим, что после изменения метрики будет $\operatorname{mesh}\left(M_{k}\right)=\frac{D_{i_{k}}}{\lambda_{i_{k}}^{r_{k}}}>k$. Тогда в силу произвольности $k$ лемма 6.1 влечет, что as $\operatorname{dim} X \leqslant m-1$, что противоречит предположению as $\operatorname{dim} X=m$.

Итак, пусть $\frac{D_{i}}{\lambda_{i}}<b$ для всех $i$. По индукции мы определим последовательность $m$-симплексов $\Delta_{i}^{m} \subset K_{i}$ так, что множества $A_{i}=\varphi_{i}^{-1}\left(\Delta_{i}^{m}\right)$ дизъюнктны и $L\left(S\left(\left.\varphi_{i}\right|_{\varphi_{i}^{-1}\left(\Delta^{m}\right)}\right)\right) \geqslant \frac{D_{i}}{4 b}$. Если $\Delta_{1}, \ldots, \Delta_{i}$ уже определены, мы возьмем $r_{0}$ таким, что $\bigcup_{l=1}^{i} A_{l} \subset \varphi_{i+1}^{-1}\left(B_{r_{0}}\left(\varphi_{i+1}\left(x_{0}\right)\right)\right)$. Всегда найдется $r>r_{0}$ с $\lambda_{i+1}^{r}>\frac{D_{i+1}}{2 b}$. Выберем $\Delta_{i+1}^{m}$ лежашим в $K_{i+1} \backslash \operatorname{Int} B_{r}\left(\varphi_{i+1}\left(x_{0}\right)\right)$ с $L\left(S\left(\left.\varphi_{i+1}\right|_{\varphi_{i+1}^{-1}\left(\Delta^{m}\right)}\right)\right) \geqslant \frac{D_{i+1}^{2 b}}{4 b}$. 
Пусть $p_{i}: \Delta_{i}^{m} \rightarrow \Delta^{m}$ обозначает линейное отображение в стандартньй единичньй симплекс. Таким образом, отображение $p_{i}-D_{i}$-сжимающее. Обозначим $A=$ $\bigcup_{i=1}^{\infty} A_{i}$. Рассмотрим отображение $f: A \rightarrow \Delta^{m}$, определенное по формуле $f=$ $\left.\bigcup_{i=1}^{\infty} p_{i} \circ \varphi_{i}\right|_{A_{i}}$. Так как $D_{i}$ стремится к бесконечности, то $\operatorname{Var}_{R} f(x)$ стремится к нулю для любого $R$. Следовательно, отображение $f$ можно продолжить на корону Хигсона $\bar{f}: \bar{A}=A \cup \nu A \rightarrow \Delta^{m}$. Покажем, что ограничение $\left.\bar{f}\right|_{\nu A}: \nu A \rightarrow \Delta^{m}$ сушественно. Предположим, что оно несущественно. Пусть $f^{\prime}: \nu A \rightarrow \partial \Delta^{m}$ обозначает выметание отображения $\left.\bar{f}\right|_{\nu A}$ на границу $\partial \Delta^{m}$. Обозначим $\partial A_{i}=\varphi_{i}^{-1}\left(\partial \Delta^{m}\right)$. Рассмотрим отображение $g=\left.f^{\prime} \cup f\right|_{\bigcup_{i=1}^{\infty}} \partial A_{i}: \bigcup \partial A_{i} \cup \nu A \rightarrow \partial \Delta^{m}$. Отображение $g$ непрерьвно, так как оно склеено из двух непрерьвных отображений $f^{\prime}$ и $\left.\bar{f}\right|_{\bar{U} A_{i}}$, совпадающих на пересечении $\bar{f}^{-1}\left(\partial \Delta^{m}\right) \cup \nu A$. Так как $\partial \Delta^{m} \in A N E$, сушествует продолжение $\bar{g}: N \rightarrow \partial \Delta^{m}$ отображения $g$ с замкнутого подмножества $\bigcup \partial A_{i} \cup \nu A$ в пространстве $\bar{A}$ на окрестность $N$. Тогда $A_{i} \subset N$ для больших $i$. Положим $g_{i}=\left.\bar{g}\right|_{A_{i}}$. Так как $\bigcup_{i \geqslant k} g_{i}$ продолжается на $\nu A$ (посредством $\bar{g}$ ), имеем $\lim _{i \rightarrow \infty} L\left(\left\{g_{i}\right\}\right)=0$. С другой стороны,

\section{Противоречие.}

$$
L\left(\left\{g_{i}\right\}\right) \geqslant L\left(S\left(\left.p_{i} \circ \varphi_{i}\right|_{\varphi_{i}^{-1}\left(\Delta^{m}\right)}\right)\right) \geqslant \frac{1}{D_{i}} \frac{D_{i}}{4 b}=\frac{1}{4 b} .
$$

ПРЕДЛОЖЕНИЕ 6.3. Для метрического пространства $X$ неравенство as $\operatorname{dim}_{*} X \leqslant m$ равносильно тому, что для всякой стремящейся $к$ бесконечности функиии $f: \mathbb{R}_{+} \rightarrow \mathbb{R}_{+}$существует отображение $\varphi: X \rightarrow K$ в локально-конечный кусочно-евклидов симплициальный комплекс мелкости один и размерности $m$ mакое, что $\lim _{\Delta \subset K} L\left(\left\{\left.\varphi\right|_{\varphi^{-1}(\Delta)}\right\}\right)=0 u \operatorname{diam} \varphi^{-1}(\Delta)<\min _{x \in \varphi^{-1}(\Delta)} f(\|x\|) \partial \Omega_{\text {я }}$ всех симплексов $\Delta$, лежащих вне некоторого компактного множества $C \subset K$.

Доказательство легко выводится из предложения 4.4.

Лемма 6.4. Ecлu as $\operatorname{dim}_{*} X<\infty, m o \operatorname{as} \operatorname{dim}_{*} X=\operatorname{dim} \nu X$.

ДоказАТЕЛьство. Пусть as $\operatorname{dim}_{*} X=m$, покажем, что $\operatorname{dim} \nu X \geqslant m$. Утверждается, что сушествует совершенное отображение $\varphi: X \rightarrow K$ на комплекс мелкости один и размерности $m$ и последовательность (различных) $m$-симплексов $\Delta_{k}^{m} \subset K$ с $L\left(S\left\{\left.\varphi\right|_{\varphi^{-1}\left(\Delta_{k}^{m}\right)}\right\}\right)>a$ для некоторого положительного числа $a$. Предположим противное. Это означает, что всякое отображение $\varphi: X \rightarrow K$ может быть вытеснено на $(m-1)$-мерный остов, $\psi: X \rightarrow K^{(m-1)}$, причем вытеснение обладает свойством $\lim _{\sigma \subset K^{(m-1)}} L\left(\left\{\left.\psi\right|_{\psi^{-1}(\sigma)}\right\}\right)=0$. Если показать, что вытеснение $\psi$ может быть взято с наперед заданным контролем $\operatorname{diam} \psi^{-1}(\sigma)<\min _{x \in \psi^{-1}(\sigma)} f(\|x\|)$, то получится, что as $\operatorname{dim}_{*} X \leqslant m-1$, что противоречит предположению as $\operatorname{dim}_{*} X=m$. Можем предположить, что контролирующая функция $f$ монотонна и $f(t) \leqslant \frac{1}{2} t$. Тогда достаточно взять $\varphi: X \rightarrow K$, подчиненное функции $g(t)=\frac{1}{2} f\left(\frac{1}{2} t\right)$.

Пусть $\varphi: X \rightarrow K$ и $\left\{\Delta_{k}^{m}\right\}$ такие, как утверждается. Обозначим $A_{k}=\varphi^{-1}\left(\Delta_{k}^{m}\right)$, $A=\bigcup A_{k}$. Пусть $p_{k}: \Delta_{k}^{m} \rightarrow \Delta^{m}$ означает отождествление со стандартным единичным симплексом. Объединение $g=\left.\bigcup_{k} p_{k} \circ \varphi\right|_{A_{k}}: A \rightarrow \Delta^{m}$ продолжается до отображения $\bar{g}: \bar{A}=A \cup \nu A \rightarrow \Delta^{m}$. Покажем, что $\left.\bar{g}\right|_{\nu A}$ сушественно. Предположим, что существует выметание $g^{\prime}: \nu A \rightarrow \partial \Delta^{m}$. Тогда $g^{\prime}$ продолжается до выметания $\widetilde{g}: \bigcup_{k \geqslant l} A_{k} \cup \nu A \rightarrow \partial \Delta^{m}$. В этом случае $L\left(S\left\{\left.\varphi\right|_{A_{k}}\right\}\right) \rightarrow 0$. Противоречие с неравенством $L\left(S\left\{\left.\varphi\right|_{A_{k}}\right\}\right)>a>0$. 
СлЕДСТВИЕ. Для любого метрического пространства $X$ либо $\operatorname{as~}_{\operatorname{dim}} X=$ $\operatorname{dim} \nu X, \imath u б о$ as $\operatorname{dim}_{*} X=\operatorname{as} \operatorname{dim} X$.

ПРЕДЛОЖЕНИЕ 6.5. Для любого метрического пространства $X$ и любой непрерывной функиии $g: W \rightarrow \mathbb{R}$, заданной на замкнутом множестве $W \subset X, \partial л я$ любой последовательности $f_{n}: X \rightarrow \mathbb{R}_{+}$грубо совершенных функиий $\left.c f_{n}\right|_{W} \geqslant g$ существуют фильтрация $X=\bigcup A_{n}$ ограниченными множествами $A_{n}$ и грубо совершенная функиия $f: X \rightarrow \mathbb{R}_{+}$такая, что $\left.f\right|_{W} \geqslant g,\left.f\right|_{A_{n}} \leqslant\left. n u f\right|_{X \backslash A_{n}} \leqslant f_{n}$.

ДоказАтельство. Существует непрерьвное продолжение $\bar{g}: X \rightarrow \mathbb{R}_{+}$отображения $g$ со свойством $\bar{g} \leqslant f_{n}$ для всех $n$. Положим $A_{n}=\bigcup_{i=1}^{n+1} f_{i}^{-1}([0, n])$ и определим $f(x)=\min \left\{\max \{n-1, \bar{g}(x)\}, f_{n}(x)\right\}$ на множестве $x \in A_{n} \backslash A_{n-1}$. Заметим, что $f_{n}\left(A_{n} \backslash A_{n-1}\right)>n-1$. Действительно, если $x \in A_{n} \backslash A_{n-1}$, то $x \notin A_{n-1}=$ $\bigcup_{i=1}^{n} f_{i}^{-1}([0, n-1])$. Следовательно, $x \notin f_{n}^{-1}([0, n-1])$, т.е. $x \in f_{n}^{-1}((n-1, \infty])$ и, значит, $f_{n}(x)>n-1$. Следовательно, $f\left(A_{n} \backslash A_{n-1}\right) \geqslant n-1$. Таким образом, $f(x) \rightarrow \infty$ при $x \rightarrow \infty$, что означает грубую совершенность $f$.

Свойство $\left.f\right|_{W} \geqslant g$ выполнено автоматически.

Проверим условие $\left.f\right|_{A_{n}} \leqslant n$. Если $x \in A_{n}$, то $x \in A_{k} \backslash A_{k-1}$ при некотором $k \leqslant n$. Тогда $f(x)=\min \left\{f_{k}(x), \max \{k-1, \bar{g}(x)\}\right\} \leqslant \max \{k-1, \bar{g}(x)\}$. Так как $x \in A_{k}$, найдется $i \leqslant k+1$ такое, что $x \in f_{i}^{-1}([0, k])$. Следовательно, $\bar{g}(x) \leqslant f_{i}(x) \leqslant k$. Таким образом, $f(x) \leqslant k \leqslant n$.

Проверим, что $\left.f\right|_{X \backslash A_{n}} \leqslant f_{n}$. Если $x \in X \backslash A_{n}$, тогда $x \in A_{m} \backslash A_{m-1}$ для некоторого $m>n$. Так как $x \notin A_{m-1}$, то $f_{n}(x)>m-1$. Так как $f_{n}(x) \geqslant \bar{g}(x)$, то $f_{n}(x) \geqslant$ $\max \{m-1, \bar{g}(x)\}$. Следовательно, $f_{n}(x) \geqslant f(x)=\min \left\{f_{m}(x), \max \{m-1, \bar{g}(x)\}\right\}$.

Теорема 6.6. Для совериенного метрического пространства X следующие условия әквивалентны:

1) $\operatorname{dim}^{c} X \leqslant n$,

2) $\operatorname{dim} \nu X \leqslant n$.

ДокАЗАТЕльство. Предположим, что $\operatorname{dim}^{c} X \leqslant n$. Рассмотрим непрерывное отображение $\varphi: C \rightarrow S^{n}$ замкнутого подмножества $C \subset \nu X$ в единичную $n$-мерную сферу. Существует продолжение $\varphi^{\prime}: V \rightarrow S^{n}$ отображения $\varphi$ на замкнутую окрестность $V \supset C$ в $\bar{X}=X \cup \nu X$. Тогда $\operatorname{Var}_{R} \varphi^{\prime}(x)$ стремится к нулю при $x \rightarrow \infty$ для всякого фиксированного $R$. Рассмотрим совершенные функции $f_{n}(x)=\frac{1}{\operatorname{Var}_{n} \varphi^{\prime}(x)}$, $n \in \mathbb{N}$. Применим предложение 6.5 к последовательности $f_{n}$ при $g \equiv 0$. В результате получим грубо совершенную функцию $f: X \rightarrow \mathbb{R}_{+}$и фильтрацию $X=\bigcup A_{n}$ такие, что $f\left(A_{n}\right) \leqslant n$ и $\left.f\right|_{X \backslash A_{n}} \leqslant f_{n}$.

Согласно предложению 3.5 сушествует асимптотически липшицева функция $q: X \rightarrow \mathbb{R}_{+}$с константами $\lambda, s$ и $q \leqslant f$. Определим отображение $g: X \cap V \rightarrow \mathbb{R}^{n+1}$ формулой $g(x)=\left(q(x), \varphi^{\prime}(x)\right)$ в полярных координатах. Покажем, что $g$ - асимптотически липшищево отображение. Обозначим через $\alpha\left(z_{1}, z_{2}\right)$ угол между векторами $z_{1}$ и $z_{2}$. По теореме косинусов имеем

$$
\begin{aligned}
\|g(x)-g(y)\|^{2} & =q(x)^{2}+q(y)^{2}-2 q(x) q(y) \cos \left(\alpha\left(\varphi^{\prime}(x), \varphi^{\prime}(y)\right)\right) \\
& \leqslant(q(x)-q(y))^{2}+q(x) q(y)\left(\alpha\left(\varphi^{\prime}(x), \varphi^{\prime}(y)\right)\right)^{2} .
\end{aligned}
$$


Предположим, что $n-1 \leqslant d(x, y) \leqslant n$. Так как $q(x) \leqslant f_{n}(x)$ и $q(y) \leqslant f_{n}(y)$ на множестве $X \backslash A_{n}$, получаем

$$
\begin{aligned}
q(x) q(y)\left(\alpha\left(\varphi^{\prime}(x), \varphi^{\prime}(y)\right)\right)^{2} & \leqslant f_{n}(x) f_{n}(y)\left(\alpha\left(\varphi^{\prime}(x), \varphi^{\prime}(y)\right)\right)^{2} \\
& =\frac{\alpha\left(\varphi^{\prime}(x), \varphi^{\prime}(y)\right)}{\operatorname{Var}_{n} \varphi^{\prime}(x)} \frac{\alpha\left(\varphi^{\prime}(x), \varphi^{\prime}(y)\right)}{\operatorname{Var}_{n} \varphi^{\prime}(y)} \leqslant 1
\end{aligned}
$$

(мы рассматриваем внутреннюю метрику на $S^{n}$ ). Если $x \in A_{n}$, то $n \geqslant f(x) \geqslant q(x)$ и, следовательно,

$$
q(x) q(y)\left(\alpha\left(\varphi^{\prime}(x), \varphi^{\prime}(y)\right)\right)^{2} \leqslant n(n+\lambda d(x, y)+s) \pi^{2} .
$$

Следовательно,

$$
\|g(x)-g(y)\|^{2} \leqslant(\lambda d(x, y)+s)^{2}+((\lambda+1) d(x, y)+s+1)^{2} \pi^{2}
$$

(мы применили неравенство $n \leqslant d(x, y)+1$ )

$$
\leqslant((\lambda(\pi+1)+\pi) d(x, y)+s(\pi+1) \pi)^{2} .
$$

Аналогичное неравенство получается, если $y \in A_{n}$.

По условию $\operatorname{dim}^{c} X \leqslant n$ существует асимптотически липшищево продолжение $\bar{g}: X \rightarrow \mathbb{R}^{n+1}$ отображения $g$. Следовательно, $\bar{g}$ (однозначно) продолжается до отображения между коронами Хигсона: $\widetilde{g}: \bar{X} \rightarrow \overline{\mathbb{R}^{n+1}}$. Рассмотрим ограничение $g^{\prime}=\left.\widetilde{g}\right|_{\bar{X} \backslash \widetilde{g}^{-1}(0)}: \bar{X} \backslash \widetilde{g}^{-1}(0) \rightarrow \overline{\mathbb{R}^{n+1}} \backslash\{0\} . \quad$ Рассмотрим радиальную проекцию $\eta: \mathbb{R}^{n+1} \backslash\{0\} \rightarrow S^{n}$. Сушествует продолжение $\bar{\eta}: \overline{\mathbb{R}^{n+1} \backslash\{0\}} \rightarrow S^{n}$ на корону Хигсона. Заметим, что отображение $\eta \circ g^{\prime}$, ограниченное на $V \cap X$, совпадает с $\varphi^{\prime}$. Следовательно, $\left.\bar{\eta} \circ g^{\prime}\right|_{C}=\varphi$.

В другую сторону, предположим, что $\operatorname{dim} \nu X \leqslant n$. Возьмем произвольное совершенное асимптотически липшицево отображение $q^{\prime}: A \rightarrow \mathbb{R}^{n+1}$ замкнутого подмножества $A \subset X$. В силу леммы 3.4 существует совершенное асимптотически липшицево продолжение $q: W \rightarrow \mathbb{R}^{n+1}$ на замкнутую окрестность $W \supset A$ со свойством $\|q(a)\| \leqslant \lambda d(a, X \backslash W)+s$ при некоторых константах $\lambda, s$. Следовательно, существует продолжение $\bar{q}: \bar{W} \rightarrow \overline{R^{n+1}}$ до отображения между компактификациями Хигсона. Обозначим через $\xi: \overline{\mathbb{R}^{n+1}} \rightarrow \mathbb{R}^{n+1} \cup S^{n}=B^{n+1}$ продолжение на компактификацию Хигсона радиального гомеоморфизма $h: \mathbb{R}^{n+1} \rightarrow \operatorname{Int}\left(B^{n+1}\right)$, заданного формулой: $h(t, \theta)=\left(\frac{t}{t+1}, \theta\right)$. Заметим, что $\xi\left(\nu \mathbb{R}^{n+1}\right) \subset \partial B^{n+1}$. В силу неравенства $\operatorname{dim} \nu X \leqslant n$ сушествует продолжение $\psi: \nu X \rightarrow S^{n}$ отображения $\left.\xi \circ \bar{q}\right|_{\nu W}: \nu W \rightarrow S^{n}$. Пусть $\bar{\psi}: X \rightarrow B^{n+1}$ - продолжение отображения $\psi \cup h \circ q$. Рассмотрим функцию $g: W \rightarrow \mathbb{R}_{+}$, определенную как $g(x)=1+\|q(x)\|$. Обозначим константы для $g$, впрочем как и для $q$, из определения асимптотической липшицевости через $\bar{\lambda}_{\text {и }} \bar{s}$.

Положим $c_{n}=n \bar{\lambda}+\bar{s}$. Рассмотрим последовательность функций $f_{n}$, определенных KaK

$$
f_{n}(x)= \begin{cases}\frac{c_{n}}{\operatorname{Var}_{n} \bar{\psi}(x)}+c_{n}+1 & \text { при } x \in X \backslash W, \\ \frac{c_{n}}{\operatorname{Var}_{n}\left(\left.\bar{\psi}\right|_{W}\right)(x)}+c_{n}+1 & \text { при } x \in W .\end{cases}
$$


Заметим, что $f_{n}$ грубо совершенны. Покажем, что $g \leqslant f_{n}$ на множестве $W$. Пусть $x \in W$, предположим, что $\left.\operatorname{Var}_{n} \bar{\psi}\right|_{W}(x)=\|\bar{\psi}(x)-\bar{\psi}(y)\|$ для некоторого $y \in W$. Тогда

$$
\left.\operatorname{Var}_{n} \bar{\psi}\right|_{W}(x)=\|h(q(x))-h(q(y))\|=\left\|\frac{q(x)}{\|q(x)\|+1}-\frac{q(y)}{\|q(y)\|+1}\right\| .
$$

Обозначим через $a=\|q(x)\|$. Тогда $a-c_{n} \leqslant\|q(y)\| \leqslant a+c_{n}$ и $\|q(x)-q(y)\| \leqslant c_{n}$ в силу того, что $d(x, y) \leqslant n$. Следовательно,

$$
\begin{aligned}
\left.\operatorname{Var}_{n} \bar{\psi}\right|_{W}(x) & \leqslant \frac{1}{a+1}\|q(x)-q(y)\|+\left\|\frac{1}{a+1}-\frac{1}{\|q(y)\|+1}\right\|\|q(y)\| \\
& \leqslant \frac{c_{n}}{a+1}+\left(a+c_{n}\right)\left|\frac{1}{a+1}-\frac{1}{a-c_{n}+1}\right| \\
& =\frac{c_{n}}{a+1}+\frac{c_{n}}{a+1} \frac{1}{\left|a-c_{n}+1\right|} \leqslant \frac{c_{n}}{a-c_{n}}
\end{aligned}
$$

при условии, что $a-c_{n} \geqslant 0$. В этом случае $\frac{c_{n}}{\left.\operatorname{Var}_{n} \bar{\psi}\right|_{W}(x)} \geqslant a-c_{n}$ и, следовательно, $f_{n}(x) \geqslant a+1=g(x)$. В случае, если $a-c_{n}<0$, имеем $f_{n}(x)=\frac{c_{n}}{\operatorname{Var}_{n} \bar{\psi}_{W}(x)}+c_{n}+1$ $>a+1=g(x)$.

Применим предложение 6.5 к $\left\{f_{n}\right\}$ и $g$, получим грубо совершенную функцию $f$. Затем применим лемму 3.6 и получим совершенную асимптотически липшищеву функцию $\bar{g}: X \rightarrow \mathbb{R}_{+}$с $\bar{g} \leqslant f$ и с $\left.\bar{g}\right|_{A}=\left.g\right|_{A}$. Обозначим через $\widetilde{\lambda}$ и $\widetilde{s}$ ее грубо липшицевы константы. Определим $\widetilde{q}: X \rightarrow \mathbb{R}^{n+1}$ по формуле $\widetilde{q}(x)=\bar{\psi}(x) \bar{g}(x)$. Заметим, что $\widetilde{q}(x)=q^{\prime}(x)$ для $x \in A$. Покажем, что $\widetilde{q}$ асимптотически липшицево. Пусть $x, y \in X$, и пусть $n-1 \leqslant d(x, y) \leqslant n$. Предположим, что одна из точек $x, y$ не лежит в $W$, скажем, $y$. Тогда

$$
\begin{aligned}
\|\widetilde{q}(y)-\widetilde{q}(x)\| & \leqslant \bar{g}(y)\|\bar{\psi}(y)-\bar{\psi}(x)\|+\frac{|\bar{g}(y)-\bar{g}(x)|}{\bar{g}(y)}\|\bar{\psi}(y)\| \\
& \leqslant \bar{g}(y)\|\bar{\psi}(y)-\bar{\psi}(x)\|+|\bar{g}(y)-\bar{g}(x)|
\end{aligned}
$$

так как $1 \leqslant \bar{g}$ и $\|\bar{\psi}(y)\| \leqslant 1$. Если $y \in A_{n}$, то $\bar{g}(y) \leqslant f(y) \leqslant n \leqslant d(x, y)+1$, и мы в результате получим неравенство

$$
\|\widetilde{q}(y)-\widetilde{q}(x)\| \leqslant 2 d(x, y)+2+\widetilde{\lambda} d(x, y)+\widetilde{s}
$$

Если $y \in X \backslash A_{n}$, то $\bar{g}(y) \leqslant f_{n}(y)=\frac{n \bar{\lambda}+\bar{s}}{\operatorname{Var}_{n} \bar{\psi}(y)}+n \bar{\lambda}+\bar{s}$. Тогда

$$
\begin{aligned}
\|\widetilde{q}(y)-\widetilde{q}(x)\| & \leqslant \frac{\|\bar{\psi}(y)-\bar{\psi}(x)\|}{\operatorname{Var}_{n} \bar{\psi}(y)}(n \bar{\lambda}+\bar{s})+2(n \bar{\lambda}+\bar{s})+\widetilde{\lambda} d(x, z)+\widetilde{s} \\
& \leqslant 3(n \bar{\lambda}+\bar{s})+\widetilde{\lambda} d(x, y)+\widetilde{s} \leqslant 3((d(x, y)+1) \bar{\lambda}+\bar{s})+\widetilde{\lambda} d(x, y)+\widetilde{s}
\end{aligned}
$$


Здесь мы использовали неравенство $\frac{\|\bar{\psi}(y)-\bar{\psi}(x)\|}{\operatorname{Var}_{n} \bar{\psi}(y)} \leqslant 1$. Таким образом,

$$
\|\widetilde{q}(y)-\widetilde{q}(x)\| \leqslant(3 \bar{\lambda}+\widetilde{\lambda}) d(x, y)+(3 \bar{\lambda}+3 \bar{s}+\widetilde{s}) .
$$

Если $x$ и $y$ лежат в $W$, тогда мы используем неравенство $\frac{\|\bar{\psi}(y)-\bar{\psi}(x)\|}{\left.\operatorname{Var}_{n} \bar{\psi}\right|_{W}(y)} \leqslant 1$.

Заметим, что для геодезического пространства $X$ доказательство импликации $\operatorname{dim} \nu X \leqslant n \Rightarrow \operatorname{dim}^{c} X \leqslant n$ сушественно короче. В этом случае легко построить грубо равномерное продолжение $\bar{q}: X \rightarrow \mathbb{R}^{n+1}$ отображения $q^{\prime}: A \rightarrow \mathbb{R}^{n+1}$, которое будет автоматически асимптотически липшицевым в силу предложения 1.4 .

\section{$\S$ 7. Грубая гипотеза Новикова}

Следуюшие утверждения в асимптотической топологии влекут ту или иную версию гипотезы Новикова о высших сигнатурах.

1. ГипотеЗа Громова. Равномерно стягиваемое многообразие с ограниченной геометрией (UC\&BG) является (рационально) гиперевклидовым (гиперсферическим).

2. ГИПОТЕЗА ВАЙНБЕРГЕРА. Гомоморфизм $\delta: H^{i}(\nu X ; \mathbb{Q}) \rightarrow H_{c}^{i+1}(X ; \mathbb{Q})$ из точной последовательности пары $(\bar{X}, \nu X)$ является эпиморфизмом.

3. ГРУБАЯ ГИПОТЕЗА БАУМА-КОННА. Для любого совериенного метрического пространства $X \in U C \& B G$ гомоморфизм Ро $\mu: K_{*}^{l f}(X) \rightarrow K_{*}(C(X))$ является изоморфизмом (рациональныцм изоморфизмом, рачиональным мономорфизмом).

Эти гипотезы связаны между собой. Связь между гипотезами 1 и 2 обсуждается в [9], связь между гипотезами 2 и 3 обсуждается в [2], [3].

Напомним, что $n$-мерное многообразие $X$ называется гиперевклидовым (рационально гиперевклидовым), если сушествует морфизм $f: X \rightarrow \mathbb{R}^{n}$ в $\mathscr{A}$ степени один (не нуль). Многообразие $X$ размерности $n$ назьвается гиперсферическим (рационально гиперсферическим), если для любого числа $R>0$ сушествует совершенное сжимаюшее отображение $f: X \rightarrow S_{R}^{n}$ на стандартную сферу радиуса $R$ степени один (не нуль). Здесь "совершенное" означает, что дополнение к некоторому компактному множеству $C \subset X$ переводится посредством $f$ в одну точку.

Самым сильным результатом, поддерживающим сформулированные вьше гипотезы, является следуюшая

ТЕОРема Ю [16]. Предположим, что совершенное метрическое пространство $X \in U C \& B G$ допускает грубо равномерное вложсение в гильбертово пространство. Тогда грубая гипотеза Баума-Конна верна для $X$.

Заметим, что грубо равномерное вложение есть вложение в категории $\mathscr{A}$, рассмотренной с морфизмами Ро. Таким образом, отображение метрических пространств $f: X \rightarrow Y$ является грубо равномерным вложением, если существуют две функции $\rho_{1}, \rho_{2}: \mathbb{R}_{+} \rightarrow \mathbb{R}_{+}$, стремящиеся к бесконечности, такие, что

$$
\rho_{1}\left(d_{X}(x, y)\right) \leqslant d_{Y}(f(x), f(y)) \leqslant \rho_{2}\left(d_{X}(x, y)\right) .
$$


Даже для геодезического пространства $X$ отображение $\left.f^{-1}\right|_{f(Y)}$ не обязательно асимптотически липшицево. Таким образом, грубо равномерные вложения не обязательно вложения в смысле категории $\mathscr{A}$.

Как было замечено Хигсоном и Ро [20], данная теорема Ю влечет другую его теорему [16], утверждающую, что грубая гипотеза Баума-Конна выполняется для $X \in U C \& B G$ с $\operatorname{as} \operatorname{dim} X<\infty$. В данном параграфе мы представляем несколько результатов в том же самом направлении.

Последовательность $\left\{x_{n}\right\}$, наделенную метрикой $d$ такой, что

$$
\lim _{n \rightarrow \infty} d\left(x_{n},\left\{x_{1}, \ldots, x_{n-1}\right\}\right)=\infty,
$$

назовем 0-мерным асимптотическим полиәдром.

Заметим, что as $\operatorname{dim}\left(\left\{x_{n}\right\}, d\right)=0$ в этом случае. Также отметим, что 0 -мерньй остов любого асимптотического симплициального комплекса является 0-мерным асимптотическим полиэдром. Следуюшая лемма было доказана Дж. Ро (не опубликовано).

Лемма 7.1. Грубая гипотеза Баума-Конна выполняется для 0-мерных асимптотических полиэдров.

Лемма 7.2. Грубая гипотеза Баума-Конна выполняется для всех конечномерных асимптотических полиәдров.

ДокАЗАТЕЛЬСТво. Индукцией по размерности. Начало индукции обеспечивает лемма 7.1. Пусть $K$ будет $n$-мерным асимптотическим полиэдром. Для любого симплекса $\Delta \subset K$ обозначим через $\frac{1}{2} \Delta \subset \Delta$ в два раза меншший подобный симплекс с тем же центром. Пусть $B$ будет объединением всех $\frac{1}{2} \Delta$ в $K$. Пусть $A=\overline{K \backslash B}$. Заметим, что $A$ гомотопически эквивалентно в $\mathscr{A}(n-1)$-мерному остову $K^{(n-1)}$. Пространство $B$ гомотопически эквивалентно в $\mathscr{A}$ нульмерному асимптотическому полиэдру. Так как выполнимость грубой гипотезы Баума-Конна есть гомотопический инвариант, гипотеза выполняется для $A, B$ и $C=A \cap B$ по предположению индукции. В таком случае лемма о пяти гомоморфизмах и последовательность Майера-Виеториса влекут гипотезу для $K$.

Teорема 7.3. Предположим, что $X \in A N E(\mathscr{A}) u$ as $\operatorname{dim}_{*} X<\infty$. Тогда грубая гипотеза Баума-Конна выполняется для $X$.

Доказательство вытекает из леммы 7.2 и следующих двух лемм.

Лемма 7.4. Пусть $X \in A N E(\mathscr{A}) u$ as $\operatorname{dim}_{*} X<\infty$. Тогда $X$ гомотопически доминируется в категории $\mathscr{A}$ асимптотическим полиәдром конечной размерности.

ДоказАтельство. Так как $X \in A N E(\mathscr{A})$, сушествует совершенное отображение $\alpha: X \rightarrow \mathbb{R}_{+}$такое, что любые $\alpha$-близкие отображения в $X$ гомотопны в $\mathscr{A}$. Пусть $W$ будет окрестностью $X$ в пространстве вероятностных мер $P(X)$ такой, что сушествует ретракция $r: W \rightarrow X$ с константой Липшица $\lambda$. Рассмотрим функцию $d(x)=$ $\frac{1}{\lambda} d_{P(X)}\left(x, W \backslash r^{-1}\left(B_{\alpha(x)}(x)\right)\right)$. По условию as $\operatorname{dim}_{*} X<\infty$ сушествует сжимаюшее отображение $\varphi: X \rightarrow K_{d}$ в асимптотический полиэдр $K_{d} \subset W$ конечной размерности и с $\|\varphi\|_{A}(x)<d(x)$. Тогда

$$
d(x, r \varphi(x))=d(r x, r \varphi(x)) \leqslant \lambda d(x, \varphi(x))=\lambda\|\varphi\|_{A}(x)<d\left(x, W \backslash r^{-1}\left(B_{\alpha(x)}(x)\right)\right) .
$$


Следовательно, $r \circ \varphi$ и $1_{X} \alpha$-близки.

ЛЕмма 7.5. Предположим, что $Y$ гомотопически доминирует $X$ в $\mathscr{A}$ и предположим, что грубая гипотеза Баума-Конна выполняется для $Y$. Тогда она выполняется для $X$.

ДокАЗАтельство. Рассмотрим диаграмму:

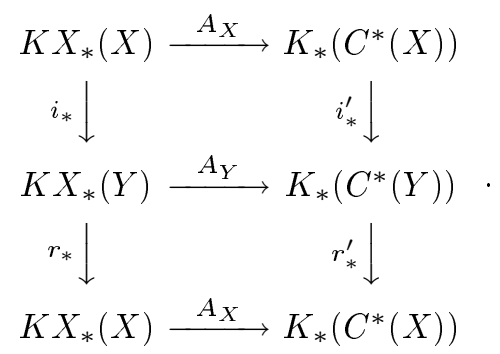

Гомоморфизмы $i_{*}, i_{*}^{\prime}, r_{*}$ и $r_{*}^{\prime}$ индуцируются морфизмами $i: X \rightarrow Y$ и $r: Y \rightarrow X$ такими, что $r \circ i$ гомотопично тождественному отображению $1_{X}$. Тогда $i_{*}$ - мономорфизм, a $r_{*}^{\prime}$ - эпиморфизм. Так как $i_{*}$ и $A_{Y}$ - мономорфизмы, то $A_{X}$ - мономорфизм. Так как $r_{*}^{\prime}$ и $A_{Y}$ - эпиморфизмы, $A_{X}-$ эпиморфизм.

Пусть $q: X \rightarrow \mathbb{R}_{+}$будет произвольной функцией, мы говорим, что два отображения $\psi_{0}, \psi_{1}: Z \rightarrow X q$-близки, если $d\left(\psi_{0}(z), \psi_{1}(z)\right)<q\left(\psi_{1}(z)\right)$. Мы говорим, что $\psi_{0}$ и $\psi_{1} q$-гомотопны, если существует гомотопия $H: Z \times I \rightarrow X$ между ними такая, что $\operatorname{diam}(H(\{z\} \times I))<q\left(\psi_{0}(z)\right)$.

ПрЕДЛОЖЕНИЕ 7.6. Предположим, что $q: X \rightarrow \mathbb{R}_{+}$есть функиия с $q(x) \leqslant$ $\frac{1}{2}\|x\|$. Тогда любые два q-близкие совершенные отображсения в $X$ совершенно гомотопньи.

ДоказАтельство. Пусть $H: Z \times I \rightarrow X$ будет $q$-гомотопией, и пусть $B_{\rho}\left(x_{0}\right)-$ шар радиуса $\rho$. Покажем, что $H$ совершенна, т.е. покажем, что $H^{-1}\left(B_{\rho}\left(x_{0}\right)\right)$ компактно. Так как отображение $\psi_{0}=\left.H\right|_{Z \times\{0\}}$ совершенно, то множество $\psi_{0}^{-1}\left(B_{2 \rho}\left(x_{0}\right)\right)$ компактно. Покажем, что $H^{-1}\left(B_{\rho}\left(x_{0}\right)\right) \subset \psi_{0}^{-1}\left(B_{2 \rho}\left(x_{0}\right)\right) \times I$. Для любого $z \in Z \backslash$ $\psi_{0}^{-1}\left(B_{2 \rho}\left(x_{0}\right)\right)$ имеем

$$
\operatorname{diam}(H(\{z\} \times I))<q\left(\psi_{0}(z)\right)<\frac{1}{2}\left\|\psi_{0}(z)\right\|=\frac{1}{2} d\left(\psi_{0}(z), x_{0}\right) .
$$

Следовательно,

$$
\begin{aligned}
d\left(x_{0}, H(\{z\} \times I)\right) & \geqslant d\left(x_{0}, \psi_{0}(z)\right)-\operatorname{diam}(H(\{z\} \times I)) \\
& \geqslant d\left(x_{0}, \psi_{0}(z)\right)-\frac{1}{2} d\left(x_{0}, \psi_{0}(z)\right)=\frac{1}{2}\left(x_{0}, \psi_{0}(z)\right)>\rho .
\end{aligned}
$$

Теорема 7.7. Для совершенного метрического пространства $X \in U C \& B G$

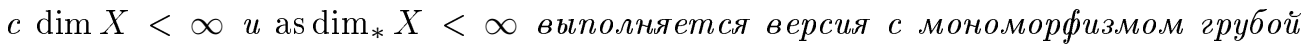
гипотези Баума-Конна. 
ДокАЗАТЕЛЬСТвО. Мы построим морфизм $\varphi: X \rightarrow K$ в конечномерньй асимптотический полиэдр $K$ и отображение $r: K \rightarrow X$ так, что композиция $r \circ \varphi$ совершенно гомотопна тождественному отображению $1_{X}$. В таком случае $r_{*} \circ \varphi_{*}=\mathrm{id}$. Так как для $U C \& B G$, пространств $K X_{*}=K_{*}^{l f}[3],[19]$, диаграмма леммы 7.5 преврашается в

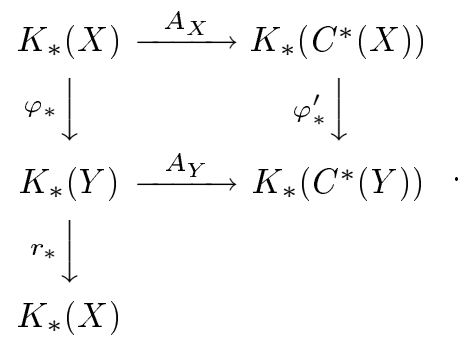

Тогда лемма 7.2 и доказательство леммы 7.5 влекут, что $A_{X}$ - мономорфизм.

Пусть $\operatorname{dim} X=m$. Так как $X \in U C$, существует совершенная монотонная функция $g_{m}: \mathbb{R}_{+} \rightarrow \mathbb{R}_{+}$такая, что для любой функции $q: X \rightarrow \mathbb{R}_{+}$любые два $q$-близкие отображения $m$-мерного пространства в $X g_{m} \circ q$-гомотопны.

Пусть $\operatorname{as~} \operatorname{dim}_{*} X=n$. Обозначим через $S: \mathbb{R}_{+} \rightarrow \mathbb{R}_{+}$функцию стягиваемости на $X$, т.е. всякий шар $B_{R}(x)$ в $X$ стягивается в точку внутри шара $B_{S(R)}(x)$ для любого $x \in X$. Определим функцию $T(t)=\frac{1}{2} S^{-1}(t / 2)$. Пусть $\bar{f}=T^{n}=T \circ \cdots \circ T$, определим $f: X \rightarrow \mathbb{R}_{+}$как $f(x)=\bar{f}\left(\frac{1}{2} g_{m}^{-1}\left(\frac{1}{4}\|x\|\right)\right)$. В силу условия as $\operatorname{dim}_{*} X \leqslant n$ сушествует сжимаюшее отображение $\varphi: X \rightarrow K_{f}$ на асимптотический полиэдр $K_{f}$ размерности $n$ с $\|\varphi\|_{U}<\frac{1}{4} f$. Можем предположить, что $\operatorname{diam}\left(\varphi^{-1}(\Delta)\right)<f(x)$ для любого $x \in \varphi^{-1}(\Delta)$. Формально данное свойство вьполняется вне некоторого компакта. В дальнейшем мы будем это игнорировать, так как ограниченные множества в категории $\mathscr{A}$ ни на что не влияют.

По индукции мы построим отображение $r: K_{f}^{(k)} \rightarrow X k$-мерного остова в $X$. Для всякой вершины $v \in K_{f}^{(0)}$ мы положим $r(v) \in \varphi^{-1}(v)$. Так как для любого 1-мерного симплекса $[u, v]$ вьполняется неравенство

$$
\begin{aligned}
\operatorname{diam} \varphi^{-1}([u, v]) & <f(r(u))=\bar{f}\left(\frac{1}{2} g_{m}^{-1}\left(\frac{1}{4}\|r(u)\|\right)\right)=T^{n}\left(\frac{1}{2} g_{m}^{-1}\left(\frac{1}{4}\|r(u)\|\right)\right) \\
& =\frac{1}{2} S^{-1}\left(\frac{1}{2} T^{n-1}\left(\frac{1}{2} g_{m}^{-1}\left(\frac{1}{4}\|r(u)\|\right)\right)\right),
\end{aligned}
$$

то существует продолжение отображения $r$ на $[u, v]$ с

$$
\operatorname{diam}(r([u, v]))<\frac{1}{2} T^{n-1}\left(\frac{1}{2} g_{m}^{-1}\left(\frac{1}{4}\|r(u)\|\right)\right) .
$$

Таким образом, $r$ определяется на $K_{f}^{(1)}$. Заметим, что для любого 2-мерного симплекса $\sigma$ с вершинами $u, v, w$ будет $\operatorname{diam}\left(r\left(\sigma^{(1)}\right)\right)<T^{n-1}\left(\frac{1}{2} g_{m}^{-1}\left(\frac{1}{4}\|r(u)\|\right)\right)$. Следовательно, сушествует продолжение отображения $r$ на $\sigma \mathrm{c}$

$$
\operatorname{diam}(r(\sigma))<\frac{1}{2} T^{n-2}\left(\frac{1}{2} g_{m}^{-1}\left(\frac{1}{4}\|r(u)\|\right)\right) .
$$


Действуя таким образом, в конце концов мы построим отображение $r: K_{f}^{(n)}=K \rightarrow X$ c $\operatorname{diam}(r(\Delta))<\frac{1}{2} g_{m}^{-1}\left(\frac{1}{4}\|r(u)\|\right)$ для любого $n$-симплекса $\Delta$. Тогда для любой точки $x \in X$ расстояние $d(x, r \varphi(x))$ может быть оценено сверху как

$$
d(x, r(u))+d(r(u), r \varphi(x))<\operatorname{diam} \varphi^{-1}(\Delta)+\operatorname{diam} r(\Delta)
$$

при $\varphi(x) \in \Delta$ и $u \in \Delta^{(0)}$. Тогда

$$
\begin{aligned}
d(x, r \varphi(x)) & <f(x)+\frac{1}{2} g_{m}^{-1}\left(\frac{1}{4}\|r(u)\|\right) \\
& \leqslant \frac{1}{2} g_{m}^{-1}\left(\frac{1}{4}\|x\|\right)+\frac{1}{2} g_{m}^{-1}\left(\frac{1}{4}(\|x\|+f(x))\right) \leqslant g_{m}^{-1}\left(\frac{1}{2}\|x\|\right) .
\end{aligned}
$$

Здесь мы использовали неравенства $T(t)<t$ и $g_{m}^{-1}(t) \leqslant t$. Следовательно, отображения $1_{X}$ и $r \circ \varphi \frac{1}{2}\|x\|$-гомотопны. Из предложения 7.6 следует, что $1_{X}$ и $r \circ \varphi$ совершенно гомотопны.

Теорема 7.8. Равномерно стягиваемое $n$-мерное многообразие $X$ асимптотической размерности $\operatorname{as} \operatorname{dim} X=n$ является гиперсферичным.

ДокАЗАТЕльство. Пусть $\Delta$ будет стандартным $n$-мерным симплексом. Пусть $c_{n}$ будет константой Липшица отображения $\nu$, переводяшего $\Delta$ в единичную полусферу $S_{+}$гомеоморфизмом внутренности $\Delta$ и сжимающего дополнение до одного $(n-1)-$ симплекса в границе $\partial \Delta$ в точку. Предположим, что дано $\varepsilon>0$. Рассмотрим сжимающее отображение $\varphi: X \rightarrow K$ в $n$-мерньй полиэдр мелкости $m>c_{n} / \varepsilon$. Рассмотрим сферу $S$ достаточно большого радиуса $R$ с центром в $s_{0}=\varphi\left(x_{0}\right)$. Очевидно, что $S$ отделяет точку $s_{0}$ от бесконечности в $K$. Рассмотрим минимальньй подкомплекс $N \subset K$, содержаший $S$. Для достаточно большого $R$ подкомплекс $N$ отделяет $s_{0}$ от $\infty$. Так как размерность $K$ равна $n,(n-1)$-мерный остов $N^{(n-1)}$ отделяет $s_{0}$ от бесконечности. Пусть $N^{\prime}$ будет границей компоненты, содержащей $s_{0}, N^{\prime} \subset N^{(n-1)}$. Тогда $M=\varphi^{-1}\left(N^{\prime}\right)$ отделяет $x_{0}$ от бесконечности в $X$. Можем предположить, что $\varphi$ - нульмерное симплициальное отображение относительно некоторой триангуляции $X$ и подразделения $K$. Тогда $M$ есть полиэдр. Граница компоненты, содержащей $x_{0}$, определяет цикл $c$, являющийся образующей $(n-1)$-мерной групшы гомологий пространства $X \backslash\left\{x_{0}\right\}$. Пусть $C=\operatorname{supp}(c)$. Так как $X \in U C$, сушествует "аппроксимативное" поднятие $\alpha: K \rightarrow X$ отображения $\varphi$. При достаточно больших $R$ отображение $\left.\alpha \circ \varphi\right|_{C}$ гомотопно тождественному $1_{C}$ в $X \backslash\left\{x_{0}\right\}$. Следовательно, $\left(\left.\varphi\right|_{C}\right)_{*}(c) \neq 0$. Тогда существует $(n-1)$-мерньй симплекс $\sigma$ в $N^{\prime}$ такой, что $\left.\varphi\right|_{\varphi^{-1}(\sigma)}$ существенно. Более того, небольшое исследование показывает, что гомоморфизм

$$
\begin{aligned}
\mathbb{Z}=H_{n-1}(C) \rightarrow H_{n-1}(M) & \rightarrow H_{n-1}\left(M, \varphi^{-1}\left(N^{\prime} \backslash \operatorname{Int}(\sigma)\right)\right) \\
& \rightarrow H_{n-1}\left(N^{\prime}, N^{\prime} \backslash \operatorname{Int}(\sigma)\right)=H_{n-1}(\sigma, \partial \sigma)=\mathbb{Z}
\end{aligned}
$$

имеет степень один. Обозначим через $W$ звезду симплекса $\sigma$ в $K$, т.е. $W$ является объединением всех симплексов, содержаших $\sigma$. Определим $\frac{c_{n}}{m}$-липшицево отображение $\psi: W \rightarrow S^{n}$ в единичную сферу, используя отображение $\nu$, так, что все $n$-симплексы $\Delta \supset \sigma$, содержащиеся в компоненте $s_{0}$, отображаются в нижнюю полусферу, а все другие $n$-симплексы $\Delta \supset \sigma$ отображаются в верхнюю полусферу. Тогда отображение $\bar{\psi}: X \rightarrow S^{n}$, определенное как $\psi \circ \varphi$ на $\varphi^{-1}(W)$ и как постоянное отображение на $X \backslash \varphi^{-1}(W)$, является $\varepsilon$-липшицевым отображением степени один. 
ТЕОРема 7.9. Грубая гипотеза Баума-Конна выполняется для любого метрического пространства X слабо растущей размерности.

Доказательство опирается на теорему Ю и следующую лемму, являющуюся комбинацией результатов Хигсона-Ро и Г. Ю [20], [16]. Заметим, что вероятностная мера $\mu$ на дискретном пространстве $Z$ может быть рассмотрена как функция $\mu: Z \rightarrow$ $[0,1]$ с $\sum_{z \in Z} \mu(z)=1$. Носитель $\operatorname{supp}(\mu)$ меры $\mu$ состоит из тех $z \in Z$, где $\mu \neq 0$. Обозначим через $\|\cdot\|_{1} l_{1}$-норму на пространстве функций.

ЛЕмма 7.10 [21], [16]. Предположим, что дискретное пространство $Z$ ограниченной геометрии обладает последовательностью отображений $a^{n}: Z \rightarrow$ $P(Z)$ в вероятностные мери такой, что

(1) для любого $n$ существует $R$ такое, что $\operatorname{supp}\left(a^{n}(z)\right) \subset B_{R}(z)$;

(2) для любого $K>0 \lim _{n \rightarrow \infty} \sup _{d(z, w)<K}\left\|a^{n}(z)-a^{n}(w)\right\|_{1}=0$.

Тогда существует грубо равномерное вложсение $Z$ в гильбертово пространство.

ДОКАЗАТЕЛЬСТВО ТЕОРЕМЫ 7.9. Мы собираемся применить лемму 7.10 и теорему Ю. Достаточно вложить в гильбертово пространство дискретную $\varepsilon$-сеть $Z \subset X$. Для любого $L$ сушествует открытое покрытие $\mathscr{U}$ пространства $Z$ с числом Лебега $L$ порядка $d(L)$. Пусть $\mathscr{U}=\left\{U_{i}\right\}_{i \in J}$. Определим $\varphi_{i}(x)=\frac{d\left(x, Z \backslash U_{i}\right)}{\sum_{i \in J} d\left(x, Z \backslash U_{j}\right)}$. Обозначим $\Sigma_{x}=\sum_{i \in J} d\left(x, Z \backslash U_{j}\right)$. Для любого $i$ фиксируем $y_{i} \in U_{i}$. Определим меру $a^{L}(z)=\sum_{i \in J} \varphi_{i}(z) \delta_{y_{i}} \in P(Z)$. Заметим, что $\operatorname{supp}\left(a^{L}(z)\right)=\left\{y_{i} \mid \varphi_{i}(z) \neq 0\right\}=\left\{y_{i} \mid\right.$ $\left.z \in U_{i}\right\} \subset\left\{z^{\prime} \in Z \mid d\left(z, z^{\prime}\right)<R\right\}$, где mesh $\mathscr{U}<R$. Таким образом, условие (1) леммы 7.10 вьполнено.

Чтобы проверить условие (2), заметим, что

$$
\begin{aligned}
\left\|a^{L}(z)-a^{L}(w)\right\|_{1}= & \left\|\sum_{i \in J} \varphi_{i}(z) \delta_{y_{i}}-\sum_{i \in J} \varphi_{i}(w) \delta_{y_{i}}\right\|_{1} \\
= & \left\|\sum_{i \in J}\left(\varphi_{i}(z)-\varphi_{i}(w)\right) \delta_{y_{i}}\right\|_{1} \\
= & \sum_{i \in J}\left|\varphi_{i}(z)-\varphi_{i}(w)\right|=\sum_{i \in J}\left|\frac{d\left(z, Z \backslash U_{i}\right)}{\Sigma_{z}}-\frac{d\left(w, Z \backslash U_{i}\right)}{\Sigma_{w}}\right| \\
\leqslant & \sum_{i \in J}\left|\frac{d\left(z, Z \backslash U_{i}\right)}{\Sigma_{z}}-\frac{d\left(w, Z \backslash U_{i}\right)}{\Sigma_{z}}\right| \\
& +\sum_{i \in J}\left|\frac{d\left(w, Z \backslash U_{i}\right)}{\Sigma_{z}}-\frac{d\left(w, Z \backslash U_{i}\right)}{\Sigma_{w}}\right| \\
= & \frac{1}{\Sigma_{z}} \sum_{i \in J}\left|d\left(z, Z \backslash U_{i}\right)-d\left(w, Z \backslash U_{i}\right)\right| \\
& +\sum_{i \in J} d\left(w, Z \backslash U_{i}\right)\left|\frac{1}{\Sigma_{z}}-\frac{1}{\Sigma_{w}}\right| \\
\leqslant & \frac{2 d(L)}{\Sigma_{z}} d(z, w)+\Sigma_{w}\left|\frac{1}{\Sigma_{z}}-\frac{1}{\Sigma_{w}}\right|
\end{aligned}
$$




$$
\begin{aligned}
& =\frac{2 d(L)}{\Sigma_{z}} d(z, w)+\frac{\left|\Sigma_{w}-\Sigma_{z}\right|}{\Sigma_{z}} \\
& \leqslant \frac{2 d(L)}{L} d(z, w)+\frac{2 d(L)}{L} d(z, w) \leqslant 4 K \frac{d(L)}{L}
\end{aligned}
$$

стремится к 0 при $L \rightarrow \infty$.

В классической теории размерности бесконечномерных пространств имеется специальный класс пространств со свойством С. Пространства со свойством С близки по своим качествам к конечномерньм [21]. Ниже мы определяем асимптотическое свойство C.

ОПРЕДЕЛЕНИЕ. Метрическое пространство $X$ обладает асимптотическим свойством $C$, если для всякой последовательности чисел $R_{1} \leqslant R_{2} \leqslant R_{3} \leqslant \ldots$ сушествует конечная последовательность равномерно ограниченных семейств открытых подмножеств $\left\{\mathscr{U}_{i}\right\}_{i=1}^{k}$ такая, что объединение $\bigcup_{i=1}^{k} \mathscr{U}_{i}$ является покрытием $X$ и каждое семейство $\mathscr{U}_{i}$ является $R_{i}$-дизъюнктным.

Следующая теорема близка к теореме 7.9 .

ТЕОрема 7.11. Грубая гипотеза Баума-Конна выполняется для метрических пространств с асимптотическим свойством $C$.

ДокАЗАТЕльство. Применим лемму 7.10 и теорему Ю. Определим отображение $a^{n}: X \rightarrow P(X)$ следующим образом. Мы предполагаем, что $X$ дискретно. Пусть $R_{i}=n^{i}$, и пусть $\mathscr{U}_{1}, \ldots, \mathscr{U}_{k}$ - последовательность $2 R_{i}$-дизъюнктных семейств из определения свойства С. Для любого открытого множества $U_{j}^{i} \in \mathscr{U}_{i}$ зафиксируем точку $x_{j}^{i} \in U_{j}^{i}$. Определим

$$
\varphi_{j}^{i}(x)= \begin{cases}\frac{R_{i}}{2}, & \text { если } x \in U_{j}^{i}, \\ \max \left\{0, \frac{R_{i}}{2}-d\left(x, U_{j}^{i}\right)\right\}, & \text { если } x \notin U_{j}^{i},\end{cases}
$$

и положим $b^{n}(x)=\sum_{i, j} n^{k-i+1} \varphi_{j}^{i}(x) \delta_{x_{j}^{i}}$. Положим $a^{n}(x)=\frac{b^{n}(x)}{\left\|b^{n}(x)\right\|_{1}}$. Заметим, что для любого $x \in X$ и для любого $i$ существует от силы одно $j=j_{x}(i)$ такое, что $\varphi_{j}^{i}(x) \neq 0$. В таком случае $\operatorname{supp} a^{n}(x) \subset B_{r}(x)$, где $r=\max \left\{\operatorname{diam} \mathscr{U}_{i}+2 R_{i}\right\}$. Таким образом, условие (1) леммы 7.10 проверено.

Для проверки условия (2) сначала покажем, что $\left\|b_{x}^{n}\right\|_{1} \geqslant \frac{n^{k+1}}{2}$ для любого $x$. Действительно, $\left\|b_{x}^{n}\right\|_{1}=\sum_{i} n^{k-i+1}\left|\varphi_{j(i)}^{i}(x)\right|$. Сушествует $i$ такое, что $x \in U_{j(i)}^{i}$. Тогда $\varphi_{j(i)}^{i}(x)=\frac{R_{i}}{2}$. Следовательно, $\left\|b_{x}^{n}\right\|_{1} \geqslant n^{k-i+1} \frac{R_{i}}{2}=\frac{n^{k+1}}{2}$. Не теряя общности, 
можно считать, что $\left\|b_{y}^{n}\right\|_{1} \geqslant\left\|b_{x}^{n}\right\|_{1}$. В таком случае

$$
\begin{aligned}
\left\|b_{x}^{n}-\right\| b_{x}^{n}\left\|_{1} a_{y}^{n}\right\|_{1} \leqslant & \left\|\frac{\left\|b_{x}^{n}\right\|_{1}}{\left\|b_{y}^{n}\right\|_{1}} b_{y}^{n}-b_{y}^{n}\right\|_{1}+\left\|b_{y}^{n}-b_{x}^{n}\right\|_{1} \\
= & \left\|b_{y}^{n}\right\|_{1} \frac{\|\| b_{x}^{n}\left\|_{1}-\right\| b_{y}^{n} \|_{1} \mid}{\left\|b_{y}^{n}\right\|_{1}}+\left\|b_{x}^{n}-b_{y}^{n}\right\|_{1} \leqslant 2\left\|b_{x}^{n}-b_{y}^{n}\right\|_{1} \\
= & 2\left\|\sum_{i} n^{k-i+1} \varphi_{j_{x}(i)}^{i}(x) \delta_{x_{j x}^{i}(i)}-\sum_{i} n^{k-i+1} \varphi_{j_{y}(i)}^{i}(y) \delta_{x_{j_{y}(i)}^{i}}\right\|_{1} \\
\leqslant & 2\left\|\sum_{i, j=j_{x}(i)=j_{y}(i)} n^{k-i+1}\left(\varphi_{j(i)}^{i}(x)-\varphi_{j(i)}^{i}(y)\right) \delta_{x_{j(i)}^{i}}\right\|_{1} \\
& +2\left\|\sum_{i, j_{x}(i) \neq j_{y}(i)} n^{k-i+1} \varphi_{j_{x}(i)}^{i}(x) \delta_{x_{j x}^{i}(i)}\right\|_{1} \\
& +2\left\|_{i, j_{x}(i) \neq j_{y}(i)} n^{k-i+1} \varphi_{j_{y}(i)}^{i}(y) \delta_{x_{j y}^{i}(i)}\right\|_{1} \\
\leqslant & 2 \sum_{i} n^{k-i+1\left|\varphi_{j(i)}^{i}(x)-\varphi_{j(i)}^{i}(y)\right|+4 \sum_{i} n^{k-i+1} d(x, y) .}
\end{aligned}
$$

Здесь мы воспользовались тем, что при $j_{x}(i) \neq j_{y}(i) d(x, y) \geqslant R_{i}$, a $\varphi_{j}^{i} \leqslant \frac{R_{i}}{2}$ по определению.

Заметим, что $\left|\varphi_{j(i)}^{i}(x)-\varphi_{j(i)}^{i}(y)\right| \leqslant d(x, y)$. Тогда

$$
\sum_{i} n^{k-i+1}\left|\varphi_{j(i)}^{i}(x)-\varphi_{j(i)}^{i}(y)\right| \leqslant \sum_{i=1}^{k} n^{k-i+1} d(x, y)=n \frac{n^{k}-1}{n-1} d(x, y) .
$$

Тогда $\left\|b_{x}^{n}-\right\| b_{x}^{n}\left\|_{1} a_{y}^{n}\right\|_{1} \leqslant 6 n \frac{n^{k}-1}{n-1} d(x, y)$. В таком случае

$$
\begin{aligned}
\left\|a_{x}^{n}-a_{y}^{n}\right\|_{1} & =\frac{1}{\left\|b_{x}^{n}\right\|_{1}}\left\|b_{x}^{n}-\right\| b_{x}^{n}\left\|_{1} a_{y}^{n}\right\|_{1} \\
& \leqslant \frac{6}{\left\|b_{x}^{n}\right\|_{1}} n \frac{n^{k}-1}{n-1} d(x, y) \leqslant \frac{6 n\left(n^{k}-1\right)}{\left(\frac{n^{k+1}}{2}\right)^{n-1}} d(x, y) \leqslant \frac{12 d(x, y)}{n-1} .
\end{aligned}
$$

Тогда для $x$ и $y$ с $d(x, y) \leqslant K$

$$
\lim _{n \rightarrow \infty} \frac{12 d(x, y)}{n-1}=0
$$

\section{$\S$ 8. Принцип спуска и корона Хигсона}

Принцип спуска - это утверждение, что оригинальная гипотеза Новикова о высших сигнатурах для геометрически конечных групп $(K(\Gamma, 1)-$ конечньй комплекс) следует из грубых аналогов. Основной грубый аналог гипотезы Новикова - это грубая гипотеза Баума-Конна, упомянутая в предыдушем параграфе. Здесь мы рассмотрим несколько других утверждений в грубой категории, также влекущих гипотезу 
Новикова. Обозначим через $X$ универсальное накрытие конечного асферического полиэдра $B Г$, снабженное метрикой, поднятой из $B Г$. Каждое из следуюших четырех утверждений влечет гипотезу Новикова для групшы $Г$.

(CPI) [22]. Существует эквивариантная рачионально ациклическая метризуемая компактификация $\widehat{X}$ пространства $X$ такая, что действие группь $\Gamma$ мало на бесконечности.

(CPII) [23]. Существуют эквивариантная рационально ациклическая (возможно неметризуемая) компактификачия $\widehat{X}$ пространства $X$ и семейство покрытий $\alpha$ нароста $Y=\widehat{X} \backslash X$ ограниченно насыщенными множсествами такие, что проекиия $Y \rightarrow \lim N(\alpha)$ в обратный предел нервов системы $\alpha$ индуцирует изоморфизм $H_{*}(Y ; \overleftarrow{Q}) \rightarrow H_{*}(\lim N(\alpha) ; \mathbb{Q})$.

$(\mathrm{FW})[24],[9]$. Граничный гомоморфизм $\delta: H^{n-1}(\nu X ; \mathbb{Q}) \rightarrow H_{c}^{n}(X ; \mathbb{Q})$ является әквивариантно расщепляющейся сюрвекиией.

(HR) [2]. Существует әквивариантная рационально ациклическая компактификация $X$ пространства $X$, подчиненная компактификачии Хигсона.

Действие группы $\Gamma$ мало на бесконечности для данной компактификации $\bar{X}$ пространства $X=E \Gamma$, если для любого $x \in \bar{X} \backslash X$ и любой окрестности $U$ точки $x$ в $\bar{X}$ для любого компактного $C$ существует менњшая окрестность $V$ такая, что условие $g(C) \cap V \neq \varnothing$ влечет $g(C) \subset U$ для всех $g \in \Gamma$.

Открытое множество $U \subset Y=\widehat{X} \backslash X$ назьвается ограниченно насыщенным, если для всякого замкнутого множества $C \subset \widehat{X}$ с $C \cap Y \subset U$ замыкание $r$-окрестности $N_{r}(C \cap X)$ удовлетворяет условию $\overline{N_{r}(C \cap X)} \cap Y \subset U$.

Мы рассматриваем Г-инвариантную метрику на $X=E \Gamma$. Так как $B \Gamma-$ конечный комплекс, корона Хигсона пространства $X$ не зависит от выбора инвариантной метрики и совпадает с короной Хигсона группы $\Gamma$.

ПРЕДЛОЖЕНИЕ 8.1 [9]. Действие группы Г на Х мало на бесконечности для компактификации $\bar{X}$, если и только если компактификация $\bar{X}$ подчинена компактификации Хигсона.

Предположим, что $\Gamma$ - конечно порожденная группа с метрикой $d(a, b)=l\left(a^{-1} b\right)$, где $l(w)$ означает минимальную длину слова, представляюшего $w$ в алфавите, состоящем из фиксированного конечного множества образующих. Группа $Г$ действует на себе изометриями $g: \Gamma \rightarrow \Gamma$ по формуле $g(a)=g a$. Данное действие назьвается леBbl.M.

ПРЕДЛОЖЕНИЕ 8.2. Левое действие группь Г на себе продолжается до действия на компактификации Хигсона.

ДокАЗАТЕльство. Любая изометрия $g: \Gamma \rightarrow \Gamma$ продолжается на корону Хигсона до непрерьвного отображения $\bar{g}: \bar{\Gamma} \rightarrow \bar{\Gamma}$. Из этого вытекает, что все действие $\Gamma$ продолжается на $\bar{\Gamma}$.

ЛЕмма 8.3. Предположим, что $X=$ ЕГ как и выше. Тогда следующие условия эквивалентны:

(1) граничный гомоморфизм $\delta: H^{n-1}(\nu X ; \mathbb{Q}) \rightarrow H_{c}^{n}(X ; \mathbb{Q})$ является эквивариантно расщепляющейся сюрвекиией; 
(2) существует әквивариантная компактификация $\widehat{X}$, подчиненная компактификации Хигсона, такая, что граничный гомоморфизм $\widehat{\delta}: H^{n-1}(\widehat{X} \backslash$ $X ; \mathbb{Q}) \rightarrow H_{c}^{n}(X ; \mathbb{Q})$ есть эквивариантно расщепляющаяся сюргекиия;

(3) существует метризуемая әквивариантная компактификация $\widehat{X}$, подчиненная компактификации Хигсона, такая, что граничный гомоморфизм $\widehat{\delta}: H^{n-1}(\widehat{X} \backslash X ; \mathbb{Q}) \rightarrow H_{c}^{n}(X ; \mathbb{Q})$ есть әквивариантно расщепляющаяся сюрвекиия;

(4) существует әквивариантная метризуемая компактификация $\widehat{X}$, подчиненная компактификации Хигсона, такая, что граничный гомоморфизм $H_{*}^{l f}(X ; \mathbb{Q}) \rightarrow H_{*-1}^{s}(\widehat{X} \backslash X ; \mathbb{Q})$ есть эквивариантно расщепляющийся мономорфизм.

Здесь $H_{*}^{s}$ означает гомологии Стинрода.

ДокАЗАТЕльство. (1) $\Rightarrow(2)$ следует из предложения 8.2.

$(2) \Rightarrow(1)$. Предположим, что $\xi: \bar{X} \rightarrow \widehat{X}$ есть отображение компактификации Хигсона, $\left.\xi\right|_{X}=1_{X}$. Отображение $\xi$ эквивариантно, так как оно эквивариантно на всюду плотном подмножестве. Пусть $s^{\prime}: H_{c}^{n}(X ; \mathbb{Q}) \rightarrow H^{n-1}(\widehat{X} \backslash X ; \mathbb{Q})$ является эквивариантным расщеплением гомоморфизма $\widehat{\delta}$. Тогда $\left(\left.\xi\right|_{\nu X}\right)^{*} \circ s^{\prime}$ будет эквивариантньм расщеплением гомоморфизма $\delta$.

$(3) \Rightarrow(2)$. Очевидно.

$(3) \Leftrightarrow(4)$. Данная эквивалентность представляет собой стандартную двойственность (векторных пространств) между гомологиями и когомологиями с коэффициентами в поле.

Импликация $(2) \Rightarrow(3)$ является очевидной для тех, кто знаком с так называемой спектральной техникой Щепина. Ниже мы приводим детали.

Направленным множеством называется частично упорядоченное множество $A$, в котором для любых двух $\alpha, \beta \in A$ сушествует $\gamma \in A$ такое, что $\gamma \geqslant \alpha$ и $\gamma \geqslant \beta$. Направленное множество $\sigma$-полно, если у всякой счетной цепи $C \subset A$ имеется супремум $\sup C \in A$. Подмножество $B \subset A \sigma$-полного направленного множества $A$ назьвается $\sigma$-замкнутым, если для любой счетной цепи $C$ в $B$ будет $\sup C \in B$. Подмножество $B \subset A$ называется конфинальным, если для любого $\alpha \in A$ найдется $\beta \in B$ такое, что $\beta \geqslant \alpha$.

ПРЕДЛОЖЕНИЕ 8.4. Пересечение счетного семейства б-замкнутых конфинальных подмножсеств $\bigcap B_{i} \subset A$ является $\sigma$-замкнутым и конфинальным.

ДокАЗАТЕльство. Очевидно, что $\bigcap B_{i} \sigma$-замкнуто.

Покажем, что пересечение двух $\sigma$-замкнутых конфинальных множеств $B$ и $B^{\prime}$ конфинально. Для любого $\alpha \in A$ мы построим последовательность $\beta_{i}$ такую, что 1) $\beta_{0}=\alpha, \beta_{2 k-1} \in B$ и $\left.\beta_{2 k} \in B^{\prime} ; 2\right) \beta_{i}<\beta_{i+1}$. Тогда $\sup \left\{\beta_{i}\right\} \in B \cap B^{\prime}$ и $\sup \left\{\beta_{i}\right\} \geqslant \alpha$.

Затем мы построим последовательность $\alpha_{i}$ такую, что 1) $\left.\alpha_{i} \leqslant \alpha_{i+1}, 2\right) \alpha_{i} \in \bigcap_{k}^{i} B_{k}$, где $\alpha_{0} \in A$ задано. Тогда $\sup \left\{\alpha_{i}\right\} \in \bigcap B_{i}$ и $\sup \left\{\alpha_{i}\right\} \geqslant \alpha_{0}$.

Обратньй (прямой) спектр в данной категории $\mathscr{C}$, индексированньй $\sigma$-полным направленным множеством $A$, называется $\sigma$-непрерывным, если

$$
\lim _{\longleftarrow}\left\{X_{\alpha_{i}} \mid \alpha_{i} \in C\right\}=X_{\sup C} \quad\left(\lim _{\longrightarrow}\left\{H_{\alpha_{i}} \mid \alpha_{i} \in C\right\}=X_{\sup C}\right)
$$

для всякой счетной цепи $C$. 
СПЕКТРАЛЬНАЯ ТЕОРЕМА ШЕПИНА [25], [26]. Предположим, что $\left\{X_{\alpha} \mid \alpha \in A\right\}$ $u\left\{Y_{\alpha} \mid \alpha \in A\right\}$ - обратные $\sigma$-непрерывные спектры компактных метрических пространств.

1) Сушествование. Пусть $f: X=\lim \left\{X_{\alpha}\right\} \rightarrow Y=\lim \left\{Y_{\alpha}\right\}-$ непрерывное отображение. Тогда существует $\sigma$-замкнутое конфинальное подмножество $B \subset A$ и морфизм спектров $\left\{f_{\beta}\right\}_{\beta \in B}:\left\{X_{\beta} \mid \beta \in B\right\} \rightarrow\left\{Y_{\beta} \mid \beta \in B\right\}$ такой, что $f=\lim \left\{f_{\beta} \mid \beta \in B\right\}$.

2) Единственность. Пусть $\left\{f_{\alpha}\right\}_{\alpha \in A} u\left\{g_{\alpha}\right\}_{\alpha \in A}-$ два морфизма межсду обратными спектрами $\left\{X_{\alpha} \mid \alpha \in A\right\} u\left\{Y_{\alpha} \mid \alpha \in A\right\}$ maкие, что $\lim \left\{f_{\alpha}\right\}=\lim \left\{g_{\alpha}\right\}$. Тогда существует б-замкнутое конфинальное подмножество $B \subset A$ такое, что $f_{\beta}=g_{\beta}$ для всех $\beta \in B$.

СлЕДСТВИЕ. Для всякого гомеоморфизма $h: X=\lim \left\{X_{\alpha}\right\} \rightarrow Y=\lim \left\{Y_{\alpha}\right\}$ существует б-замкнутое конфинальное подмножество $B \subset A$ и изоморфизм спектров $\left\{h_{\beta}\right\}_{\beta \in B}:\left\{X_{\beta} \mid \beta \in B\right\} \rightarrow\left\{Y_{\beta} \mid \beta \in B\right\}$ mакой, что $h=\lim _{\beta}\left\{h_{\beta} \mid \beta \in B\right\}$.

ДокаЗАТЕльство. Применяем часть 2) к $h$ и $h^{-1}$, а затем применяем предложение 8.4 .

Заметим, что всякое компактное хаусдорфово пространство $X$ является обратным пределом следуюшего непрерьвного $\sigma$-спектра. Вложим $X \subset I^{C}$ в тихоновский куб и возьмем в качестве $A$ множество всех счетных подмножеств. Для любого $\alpha \in A$ определим $X_{\alpha}=\pi_{\alpha}(X)$, где $\pi_{\alpha}: I^{C} \rightarrow I^{\alpha}$ - проекция на сомножитель.

Сушествует двойственная теорема для $\sigma$-непрерывных прямых пределов счетных $C W$-комплексов [27]. В данной работе нам потребуется двойственная спектральная теорема в категории абелевых групп.

ДВОЙСТВЕННАЯ СПЕКТРАЛЬНАЯ ТЕОРЕМА. Предположим, ито $\left\{H_{\alpha} \mid \alpha \in A\right\}$ $u\left\{G_{\alpha} \mid \alpha \in A\right\}$ - прямые $\sigma$-непрерывные спектры счетных абелевих групп.

1) Сушествование. Для любого гомоморфизма $f: H=\underline{\lim }\left\{H_{\alpha}\right\} \rightarrow G=\underline{\lim }\left\{G_{\alpha}\right\}$ существует б-замкнутое конфинальное подмножсество $\vec{B} \subset A$ иморфизм спектров $\left\{f_{\beta}\right\}_{\beta \in B}:\left\{H_{\beta} \mid \beta \in B\right\} \rightarrow\left\{G_{\beta} \mid \beta \in B\right\}$ maкой, что $f=\lim \left\{f_{\beta} \mid \beta \in B\right\}$.

2) Единственность. Пусть $\left\{f_{\alpha}\right\}_{\alpha \in A} u\left\{g_{\alpha}\right\}_{\alpha \in A}-$ два морфизма спектров $\left\{H_{\alpha} \mid\right.$ $\alpha \in A\} u\left\{G_{\alpha} \mid \alpha \in A\right\} \quad c \lim \left\{f_{\alpha}\right\}=\lim \left\{g_{\alpha}\right\}$. Тогда существует $\sigma$-замкнутое конфинальное подмнохество $B \subset A$ такое, что $f_{\beta}=g_{\beta}$ для всех $\beta \in B$.

ДокАЗАТЕльство. Пусть $p_{\alpha}^{\beta}: H_{\alpha} \rightarrow H_{\beta}$ и $q_{\alpha}^{\beta}: G_{\alpha} \rightarrow G_{\beta}$ будут связьваюшими гомоморфизмами, $\alpha \leqslant \beta$. Обозначим через $p_{\alpha}=\lim _{\beta} p_{\alpha}^{\beta}: H_{\alpha} \rightarrow H$ и $q_{\alpha}=$ $\lim _{\beta} q_{\alpha}^{\beta}: G_{\alpha} \rightarrow G$.

1) Сначала покажем, что множество $M_{H}=\left\{\alpha \in A \mid \operatorname{Ker}\left(p_{\alpha}\right)=0\right\}$ конфинально в $A$. Для любого $\alpha_{0} \in A$ мы построим счетную цеп $\alpha_{0}<\alpha_{1}<\cdots$ такую, что $\operatorname{Ker}\left(p_{\alpha_{i}}^{\alpha_{i+1}}\right)=\operatorname{Ker}\left(p_{\alpha_{i}}\right)$. Это возможно в силу счетности $H_{\alpha_{i}}$ и $\sigma$-полноты $A$. Рассмотрим $\alpha=\sup \left\{\alpha_{i}\right\}$. Покажем, что $\alpha \in M_{H}$. Пусть $a \in \operatorname{Ker}\left(p_{\alpha}\right)$. Так как спектр $H_{\alpha} \quad \sigma$-непрерьвен, $a=p_{\alpha_{i}}^{\alpha}(c)$ при некоторых $i$ и $c \in H_{\alpha_{i}}$. Но тогда $c \in \operatorname{Ker}\left(p_{\alpha_{i}}\right)$. Следовательно, $p_{\alpha_{i}}^{\alpha_{i+1}}(c)=0$, и тогда $a=0$.

Заметим, что множество $M=M_{H} \cap M_{G} \sigma$-замкнуто и конфинально в $A$. Определим $B=\left\{\beta \in M \mid \exists\right.$ гомоморфизм. $f_{\beta}: H_{\beta} \rightarrow G_{\beta}$ с $\left.f \circ p_{\beta}=q_{\beta} \circ f_{\beta}\right\}$ Покажем, что множество $B$ конфинально в $A$. Пусть $\alpha \in A$. Для любого $a \in H_{\alpha}$ сушествует 
$\beta(a) \in A$ такое, что $f p_{\alpha}(a)=q_{\beta(a)}(b(a))$ для некоторого $b(a) \in G_{\beta(a)}$. Занумеруем элементы группы $H_{\alpha}: a_{1}, a_{2}, \ldots$ и построим последовательность $\alpha<\beta\left(a_{1}\right)<$ $\beta\left(a_{2}\right)<\cdots$. Положим $\gamma=\sup \left\{\beta\left(a_{i}\right)\right\}$. Определим отображение $f_{\alpha \gamma}: H_{\alpha} \rightarrow G_{\gamma}$ формулой $f_{\alpha \gamma}(a)=q_{\beta(a)}^{\gamma}(b(a))$. Очевидно, что $f \circ p_{\alpha}=q_{\gamma} \circ f_{\alpha \gamma}$. Покажем, что $f_{\alpha \gamma}$ - гомоморфизм. Равенство $f_{\alpha \gamma}\left(a+a^{\prime}\right)=f_{\alpha \gamma}(a)+f_{\alpha \gamma}\left(a^{\prime}\right)$ следует из того, что $q_{\gamma}-$ мономорфизм, и равенства

$$
q_{\gamma} f_{\alpha \gamma}\left(a+a^{\prime}\right)=f p_{\alpha}\left(a+a^{\prime}\right)=f p_{\alpha}(a)+f p_{\alpha}\left(a^{\prime}\right)=q_{\gamma} f_{\alpha \gamma}(a)+q_{\gamma} f_{\alpha \gamma}\left(a^{\prime}\right) .
$$

Определим $\alpha_{1}=\gamma$ и повторим конструкцию с $\alpha_{1}$ вместо $\alpha$. В результате мы построим счетную цеп $\alpha<\alpha_{1}<\alpha_{2}<\cdots$ и последовательность гомоморфизмов $f_{\alpha_{i} \alpha_{i+1}}: H_{\alpha_{i}} \rightarrow G_{\alpha_{i+1}}$ такую, что все квадраты коммутативны. Пусть $\bar{\alpha}=\sup \left\{\alpha_{i}\right\}$. Сушествует гомоморфизм $f_{\bar{\alpha}}: H_{\bar{\alpha}} \rightarrow G_{\bar{\alpha}}$, являюшийся пределом $f_{\bar{\alpha}}=\underline{\lim } f_{\alpha_{i} \alpha_{i+1}}$. Тогда $f \circ p_{\bar{\alpha}}=q_{\bar{\alpha}} \circ f_{\bar{\alpha}}$ и, следовательно, $\bar{\alpha} \in B$.

Очевидно, что $B \sigma$-замкнуто и что $\left\{f_{\beta}\right\}_{\beta \in B}-$ морфизм спектров.

2) Возьмем в качестве $B$ множество $M$.

ДокАЗАТЕльство $(2) \Rightarrow(3)$ лЕммы 8.3. Согласно замечанию, сделанному после спектральной теоремы Щепина, мы можем представить $\widehat{X}$ как предел $\sigma$-непрерьвного обратного спектра из компактных метрических пространств: $\widehat{X}=\lim \left\{\widehat{X}_{\alpha} ; p_{\alpha}^{\beta} \mid\right.$ $\left.\alpha \in A^{\prime}\right\}$. Легко проверить, что множество $A=\left\{\alpha A^{\prime} \mid p_{\alpha}^{-1} p_{\alpha}(X)=X\right\} \sigma$-замкнуто и конфинально в $A^{\prime}$. Для всякого элемента $\gamma \in \Gamma$ применим спектральную теорему Шепина к гомоморфизму $\gamma: \widehat{X} \rightarrow \widehat{X}$ и получим $\sigma$-замкнутое конфинальное подмножество $B_{\gamma} \subset A$ и соответствуюший морфизм спектров над $B_{\gamma}$. По предложению 8.4 множество $B=\bigcap_{\gamma \in \Gamma} B_{\gamma} \sigma$-замкнуто, конфинально. Таким образом, для любого $\alpha \in B$ пространство $\widehat{X}_{\alpha}$ обладает действием групшы $\Gamma$, причем $\widehat{X}_{\alpha}$ является эквивариантной компактификацией пространства $X$. Более того, все связьвающие проекции в обратном спектре над $B$ Г-эквивариантны. Гомоморфизм $\widehat{\delta}$ индуцирован фактор-отображением $\widehat{X} \cup \operatorname{cone}(\widehat{X} \backslash X) \rightarrow \Sigma(\widehat{X} \backslash X)$. Следовательно, групшы $H=\bigoplus_{n} H^{n}(\widehat{X} \cup \operatorname{cone}(\widehat{X} \backslash X) ; \mathbb{Q})$ и $G=\bigoplus_{n} H^{n}(\Sigma(\widehat{X} \backslash X) ; \mathbb{Q})$ представляются как прямые пределы $\left\{H_{\alpha} \mid \alpha \in B\right\}$ и $\left\{G_{\alpha} \mid \alpha \in B\right\}$ над множеством $B$. Условие (2) утверждает, что существует эквивариантное расшепление $s: H \rightarrow G$, т.е. $\widehat{\delta} \circ s=\mathrm{id}$. В силу 1) двойственной спектральной теоремы существует $B_{1} \subset B$ и последовательность гомоморфизмов $\left\{s_{\beta}: H_{\beta} \rightarrow G_{\beta} \mid \beta \in B_{1}\right\}$. В силу 2) двойственной спектральной теоремы мы можем предположить, что $\gamma^{-1} s_{\beta} \gamma=s_{\beta}$ и $\widehat{\delta}_{\beta} \circ s_{\beta}=\mathrm{id}$ для всех $\beta \in B_{1}$ и всех $\gamma \in \Gamma$. В таком случае для любого $\beta \in B_{1}$ компакт $\widehat{X}_{\beta}$ является метризуемой компактификацией пространства $X$, требуемой в условии (3).

Заметим, что гомоморфизм $H_{*}(Y ; \mathbb{Q}) \rightarrow H_{*}(\lim N(\alpha) ; \mathbb{Q})$ в $(\mathrm{CPII})$ является изоморфизмом, если система $\{\alpha\}$ конфинальна. Рассмотрим следуюшее условие:

$\left(\mathrm{CPII}^{\prime}\right)$. Существуют эквивариантная рационально ациклическая (возможсно неметризуемая) компактификация $\widehat{X}$ пространства $X$ и конфинальная система покрытий $\alpha$ нароста $Y=\widehat{X} \backslash X$ ограниченно насыщеннылми множества$\mathcal{M u}$.

Обозначим через $\left(\mathrm{CPI}^{\prime}\right)$ условие (CPI) без предположения метризуемости пространства $\widehat{X}$. 
Teopema 8.5. $\left(\mathrm{CPII}^{\prime}\right) \Leftrightarrow\left(\mathrm{CPI}^{\prime}\right) \Leftrightarrow(\mathrm{CPI}) \Leftrightarrow(\mathrm{HR}) \Rightarrow(\mathrm{FW}) \Leftarrow(\mathrm{CPII})$.

ДОКАЗАТЕЛЬСТвО. Согласно предложению 8.1 свойство компактификации быть подчиненной компактификации Хигсона равносильно тому, что действие группы мало на бесконечности. Следовательно, $\left(\mathrm{CPI}^{\prime}\right) \Leftrightarrow(\mathrm{HR})$. Точная последовательность пары $(\widehat{X}, \widehat{X} \backslash X)$ влечет импликацию $(\mathrm{HR}) \Rightarrow(\mathrm{FW})$. Очевидно, что $(\mathrm{CPI}) \Rightarrow\left(\mathrm{CPI}^{\prime}\right)$. Покажем, что $\left(\mathrm{CPI}^{\prime}\right) \Rightarrow(\mathrm{CPI})$. Пусть $\widehat{X}$ - рационально ациклическая эквивариантная компактификация пространства $X$. Представим $\widehat{X}$ как предел обратного $\sigma$-непрерьвного спектра из метрических компактов $\left\{\widehat{X}_{\alpha} \mid \alpha \in A\right\}$. Так же как при доказательстве импликации $(2) \Rightarrow(3)$ в лемме 8.3 , мы можем предположить, что каждое $\widehat{X}_{\alpha}$ есть эквивариантная компактификация пространства $X$. Рассмотрим прямой спектр $\left\{\bigoplus_{i} H^{i}\left(\widehat{X}_{\alpha} ; \mathbb{Q}\right) \mid \alpha \in A\right\}$. Пусть $M_{\oplus H^{i}} \subset A$ означает множество, определенное в доказательстве двойственной спектральной теоремы. Напомним, что по определению для любого $\alpha \in M_{\oplus H^{i}}$ ядра гомоморфизмов $p_{\alpha}^{*}: H^{i}\left(\widehat{X}_{\alpha} ; \mathbb{Q}\right) \rightarrow H^{i}(\widehat{X} ; \mathbb{Q})$ тривиальны для всех $i$. Так как $H^{i}(\widehat{X} ; \mathbb{Q})=0$, получаем, что $\widehat{X}_{\alpha}$ рационально ациклично.

Осталось показать эквивалентность $\left(\mathrm{CPII}^{\prime}\right) \Leftrightarrow\left(\mathrm{CPI}^{\prime}\right)$. Так как всякое открытое подмножество нароста компактификации, подчиненной компактификации Хигсона, ограниченно насышено, то $\left(\mathrm{CPI}^{\prime}\right) \Rightarrow\left(\mathrm{CPII}^{\prime}\right)$ очевидным образом. В обратном направлении, заметим, что компактификация $c X$ рационально ациклична. Действительно, так как система покрытий $\alpha$ конфинальна, то конфинальной будет система $g(\alpha)$ и, следовательно, $\widetilde{p}$ в вышеприведенной диаграмме - изоморфизм. Следовательно, $\widetilde{\partial}$ есть изоморфизм, и, значит, $c X$ ациклично.

Заметим, что (FW) является дальнейшим огрублением следуюшей версии грубой гипотезы Баума-Конна: рациональньй гомоморфизм $\mathrm{Po}_{*}^{l f}(X) \otimes \mathbb{Q} \rightarrow K_{*}(C(X)) \otimes \mathbb{Q}$ является эквивариантно расщепляющимся мономорфизмом.

\section{§. Открытые проблемы}

1. Размерность и корона Хигсона. Связь между асимптотическими размерностями метрического пространства и размерностями его короны Хигсона еше не до конца исследована. Проблемы данного раздела представляют особый интерес для метрических пространств ограниченной геометрии.

ПробЛема 1. Верно ли, ито $\operatorname{as} \operatorname{dim} X=\operatorname{dim} \nu X ?$

Ввиду теоремы 6.2 и [18] данная проблема может быть переформулирована в следующем виде: существует ли метрическое пространство $X$, асимптотически бесконечномерное, но $c \operatorname{dim} \nu X<\infty$ ?

Проблема 2. Существует ли метрическое пространство $X$ с $\operatorname{as} \operatorname{dim} X=\infty$ $u c$ as $\operatorname{dim}_{*} X<\infty$ ?

Положительный ответ в проблеме 2 даст отрицательный ответ в проблеме 1 . Другой вопрос по-сушеству: верно ли, что размерности $\operatorname{as} \operatorname{dim}_{*} X$ и $\operatorname{dim} \nu X$ всегда совпадают?

ПроБлема 3. Верно ли, что $\operatorname{dim}_{G} \nu X \leqslant \operatorname{as}_{\operatorname{dim}_{G}} X$ для всех метрических пространств $X$ и всех абелевых групп $G$ ? 
ПРОБЛЕмА 4. Верно ли, что $\operatorname{dim}_{\mathbb{Z}} \nu \Gamma<\infty$ для всех геометрически конечных zpynn $\Gamma$ ?

Положительный ответ в проблеме 3 влечет положительный ответ в проблеме 4 .

По аналогии можно определить асимптотическую индуктивную размерность as Ind (a также as ind).

ПроБЛЕма 5. Исследовать отношения межсду as Ind и другими размерностя$\mathcal{M} u:$ as $\operatorname{dim}, \operatorname{as} \operatorname{dim}_{*} u \operatorname{dim}^{c}$.

ПроБлема 6. Предположим, что $X \in B G$ имеет слабо растущую размерность $и$ as $\operatorname{dim} X=\infty$. Верно ли, что $\operatorname{dim} \nu X=\infty$ ?

2. Грубая проблема Александрова. Следующая проблема является асимптотическим аналогом известной проблемы Александрова о совпадении лебеговой и когомологической размерностей.

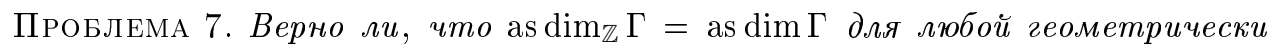
конечной әруппь?

Напомним, что спектр $\mathbb{S}=\left\{\Omega^{\infty} \Sigma^{\infty} S^{n}\right\}$ определяет стабильные когомологические группы. Стабильная когомологическая размерность $\operatorname{dim}_{\mathbb{S}} X$ может быть определена с помошью продолжений отображений в $\Omega^{\infty} \Sigma^{\infty} S^{n}$. Именно, $\left(\operatorname{dim}_{\mathbb{S}} X \leqslant n\right) \Leftrightarrow$ (для любого непрерывного отображения $f: A \rightarrow \Omega^{\infty} \Sigma^{\infty} S^{n}$ замкнутого подмножества $A \subset X$ существует продолжение на $X)$. Легко видеть, что для любого $m \in \mathbb{N}$ продолжения в $\Omega^{m} \Sigma^{m} S^{n}$ классифицируют лебегову размерность. Таким образом, размерности $\operatorname{dim}$ и $\operatorname{dim}_{\mathbb{S}}$ очень близки. Размерность $\operatorname{dim}_{\mathbb{S}}$ является обобшенной когомологической размерностью и может быть определена в терминах обобщенных когомологий $\mathbb{S}^{*}(X, A)$ пар. По аналогии можно определить асимптотический вариант as $\operatorname{dim}_{\mathbb{S}}$ стабильной когомотопической размерности.

ПроБЛЕма 8. Верно ли, что as $\operatorname{dim}_{\mathbb{S}} \Gamma=\operatorname{as} \operatorname{dim} \Gamma$ для геометрически конечных zpynn $\Gamma$ ?

Положительное решение проблем 7 и 8 влечет гипотезу Новикова. ${ }^{1}$

ПроБЛЕмА 9. Что является аналогом теоремы Эдвардса о СЕ-резолюиии в асимптотическом случае?

Напомним, что классическая теорема Эдвардса гласит, что для всякого компакта $X \mathrm{c} \mathrm{dim}_{\mathbb{Z}} X \leqslant n$ сушествует клеточно-подобное $(C E)$ отображение $f: Y \rightarrow X$ с $\operatorname{dim} Y \leqslant n$.

Следующие две проблемы относятся к обьгной топологии. Их (положительное) решение пролило бы свет на асимптотический случай.

ПРОБЛЕма 10. Верно ли, что равенство $\operatorname{dim}_{\mathbb{Z}} X=\operatorname{dim} X$ выполняется для компактных $H$-пространств?

\footnotetext{
${ }^{1}$ После того как работа была сдана в печать, М. Громов построил примеры групп, дающие отрицательное решение проблем 7 и 8.
} 
ПроБЛема 11. Верно ли, что равенство $\operatorname{dim}_{\mathbb{Z}} G=\operatorname{dim} G$ выполняется для топологических (не обязательно компактнъх) групп?

3. Абсолютные окрестностные ретракты. Напомним, что в случае компактных метрических пространств вероятностные меры $P(X)$ являются абсолютным ретрактом.

ПроБЛема 12. Верно ли, что $P(X) \in A E(\mathscr{A})$ в асимптотической категории?

Нетрудно показать, что $P(X)$ является $A E$ для конечномерных пространств, более того, $P(X)$ является $A E$ для пространств, имеюших слабо растушую размерность.

ПроБЛема 13. Верно ли, что НЕT (теорема о продолжении гомотопий) верна в полной общности в асимптотической категории?

Положительный ответ к проблеме 12 дал бы положительный ответ к проблеме 13.

ПроБЛемА 14. Доказать макроскопический аналог теоремь Веста, утверждающей, что всякий $A N E$ гомотопически әквивалентен полиэдру.

\section{4. Фрагменты микро-макро топологического словаря.}

Микро

1. Компакт, т.е. компактное метризуемое пространство

2. Аппроксимация полиэдрами по Александрову-Чеху

3. Лебеговская размерность dim

4. Характеризация размерности отображениями в сферы $S^{n}$

5. Локальная стягиваемость

6. Локальная $n$-связность

7. Окрестность

8. $A N E$

9. Полиэдр

10. Одноточечное пространство

11. Единичный интервал $[0,1]$

12. $n$-мерная сфера $S^{n}$

13. ?

14. Гомотопия

15. Когомологии

16. Чеховские гомологии, гомологии Стинрода

17. Когомологическая размерность

18. Фундаментальная группа

19. Многообразие

20. ?
Макро

Совершенное метрическое пространство ограниченной геометрии

Античеховская аппроксимация

полиэдрами

Размерность Громова as dim

Грубая размерность $\operatorname{dim}^{c}$

Равномерная стягиваемость

Равномерная $n$-связность

Асимптотическая окрестность

$A N E$

Асимптотический полиэдр;

открытьй конус над полиэдром

$\mathbb{R}_{+}$(или $\mathbb{N} \stackrel{\mathscr{C}}{\sim} \mathbb{R}_{+}$)

$\mathbb{R}_{+}^{2}$

$\mathbb{R}^{n+1}$

Гиперболическое пространство $\mathbb{H}^{n}$

Гомотопия в $\mathscr{A}$

Античеховские когомологии, когомологии Ро

Грубые гомологии

Асимптотическая когомологическая размерность

Асимптотическая фундаментальная группа, определенная с использованием $\mathbb{R}_{+}^{2}$ вместо $[0,1]$

Открытое стягиваемое многообразие?

Дискретная группа 


\section{СПИСОК ЛИТЕРАТУРЫ}

[1] Gromov M. Geometric Group Theory. V. 2: Asymptotic Invariants for Infinite Groups. Cambridge: Cambridge Univ. Press, 1993. (London Math. Soc. Lecture Note Ser. V. 182.)

[2] Roe J. Coarse Cohomology and Index Theory on Complete Riemannian Manifolds. Providence, RI: Amer. Math. Soc., 1993. (Mem. Amer. Math. Soc. V. 497.)

[3] Roe J. Index Theory, Coarse Geometry, and Topology of Manifolds. Washington, DC: Conf. Board Math. Sci., 1996. (CBMS Regional Conf. Ser. in Math. V. 90.)

[4] Gromov M. Positive curvature, macroscopic dimension, spectral gaps and higher signatures // Functional analysis on the eve of the 21st century. V. II. In honor of the eightieth birthday of I. M. Gelfand. Proceedings of a conference, held at Rutgers University, New Brunswick, NJ, USA, October 24-27, 1993 / ed. S. Gindikin et al. Boston, MA: Birkhäuser, 1996. P. 1-213. (Progr. Math. V. 132.)

[5] Yu G. The Novikov conjecture for groups with finite asymptotic dimension // Ann. of Math. (2). 1998. V. 147. №5. P. 191-226.

[6] Bestvina M. Local homology properties of boundaries of groups // Michigan Math. J. 1996. V. 43. № 1. P. 123-139.

[7] Bowers Ph., Ruane K. Boundaries of nonpositively curved groups of the form $G \times \mathbb{Z}^{n}$ // Glasgow Math. J. 1996. V. 38. №2. P. 1311-1313.

[8] Croke Ch., Kleiner B. Spaces with nonpositive curvature and their ideal boundaries // Preprint: University of Utah, 1998.

[9] Дранишников А.Н., Ферри С. О короне Хигсона-Ро // УМН. 1997. Т. 52.№5. C. $133-146$.

[10] Новиков С. П. Топологическая инвариантность рациональных классов Понтрягина // Докл. АН СССР. 1965. Т. 163. С. 298-300.

[11] Gromov M., Lawson H. B. Positive scalar curvature and the Dirac operator // Inst. Hautes Études Sci. Publ. Math. 1983. V. 58. P. 83-196.

[12] Rosenberg J. $C^{*}$-algebras, positive scalar curvature and the Novikov conjecture // Inst. Hautes Études Sci. Publ. Math. 1983. V. 58. P. 409-424.

[13] Ferry S. C., Ranicki A., Rosenberg J. (eds.) Novikov Conjectures, Index Theorems and Rigidity. V. 1, 2. Cambridge: Cambridge Univ. Press, 1995.

[14] Дранишников А.Н. Гомологическая теория размерности // УМН. 1988. Т. 43. № 4. C. $11-55$.

[15] Dranishnikov A. N., Ferry S., Weinberger S. Large Riemannian manifolds which are flexible // Preprint, 1994.

[16] Yu G. The coarse Baum-Connes conjecture for spaces which admit a uniform embedding into Hilbert space // Invent. Math. 2000. V. 139. № 1. P. 201-240.

[17] Connes A., Gromov M., Moscovici H. Group cohomology with Lipschitz control and higher signatures // Geom. Funct. Anal. 1993. V. 3. №1. P. 1-78.

[18] Dranishnikov A. N., Keesling J. E., Uspenskij V. V. On the Higson corona of uniformly contractible spaces // Topology. 1998. V. 37. № 4. P. 791-803.

[19] Higson N., Roe J. The Baum-Connes conjecture in coarse geometry // London Math. Soc. Lecture Notes. 1995. V. 227. P. 227-254.

[20] Higson N., Roe J. Amenable group actions and the Novikov conjecture // J. Reine Angew. Math. 2000. V. 519. P. 143-153.

[21] van Mill J., Mogilski J. Property C and fine homotopy equivalences // Proc. Amer. Math. Soc. 1984. V. 90. №1. P. 118-120.

[22] Carlsson G., Pedersen E. Controlled algebra and the Novikov conjecture for $K$ and $L$ theory // Topology. 1995. V. 34. P. 731-758.

[23] Carlsson G., Pedersen E. Cech homology and the Novikov conjectures for $K$ - and $L$-theory // Math. Scand. 1998. V. 82. № 1. P. 5-47.

[24] Ferry S., Weinberger S. A coarse approach to the Novikov Conjecture // London Math. Soc. Lecture Note Ser. 1995. V. 226. P. 147-163. 
[25] Chigogidze A. Inverse Spectra. Amsterdam: Elsevier, 1996.

[26] Шепин Е. В. Топология предельных пространств несчетных обратных спектров // УМН. 1976. T. 31. № 5. C. 191-226.

[27] Дранишников А.Н., Дыдак Дж. Extension dimension and extension types // Труды МИАН. 1996. Т. 212. С. 61-94.

Математический институт им. В.А. Стеклова РАН,

Поступила в редакцию Университет штата Флорида

29.12.1999

E-mail: dranish@math.ufl.edu 